\title{
EVALUATION OF STRENGTH REDUCTION FACTOR FOR CONCENTRICALLY BRACED FRAMES BASED ON NONLINEAR SINGLE DEGREE-OF-FREEDOM SYSTEMS
}

\author{
A Thesis \\ presented to \\ the Faculty of California Polytechnic State University, \\ San Luis Obispo
}

\author{
In Partial Fulfillment \\ of the Requirements for the Degree \\ Master of Science in Civil and Environmental Engineering
}

by

Ryan Michael Slein

March 2016 
(C) 2016

Ryan Michael Slein

ALL RIGHTS RESERVED 
TITLE:

AUTHOR:

DATE SUBMITTED:

March 2016
Evaluation of Strength Reduction Factor for

Concentrically Braced Frames Based on Nonlinear

Single Degree-of-Freedom Systems

Ryan Michael Slein

COMMITTEE CHAIR: Bing Qu, Ph.D.

Associate Professor of Civil Engineering

COMMITTEE MEMBER: Graham Archer, Ph.D.

Professor of Architectural Engineering

COMMITTEE MEMBER: Garrett Hall, Ph.D.

Professor of Civil Engineering 


\section{ABSTRACT}

Evaluation of Strength Reduction Factor for Concentrically Braced Frames Based on Nonlinear Single Degree-of-Freedom Systems

Ryan Michael Slein

Strength Reduction Factor (R-Factor), often referred to as Response Modification Factor, is commonly used in the design of lateral force resisting systems under seismic loading. R-Factors allow for a reduction in design base shear demands, leading to more economical designs. The reduction of strength is remedied with ductile behavior in members of proper detailing. Modern seismic codes and provisions recommend RFactors for many types of lateral force resisting systems. However these factors are independent of the system fundamental frequency and many other important system properties, resulting in factors that may result in an unfavorable seismic response. To evaluate the validity of prescribed R-Factors an extensive analytical parameter study was conducted using a FEM single degree-of-freedom Concentrically Braced Frame (CBF) under incremental dynamic analysis over a suite of ground motions. Parameters of the study include brace slenderness, fundamental frequency, increment resolution, FEM mesh refinement, effects of leaning columns, and effects of low-cycle fatigue. Results suggest that R-Factor can vary drastically for CBF systems with differing properties.

Keywords: Strength Reduction Factor, Ductility, Concentrically Braced Frame Response, Low-cycle Fatigue, Slenderness 


\section{ACKNOWLEDGMENTS}

I would like to express my deep gratitude to Professor Bing Qu. I really appreciate all of your help in furthering my academic knowledge in structural engineering while keeping the material interesting and enjoyable.

I would like to thank the professors on my thesis committee, Professor Graham Archer and Professor Garrett Hall, for taking time out of their schedule to serve on my committee.

The research reported in this thesis was supported by the U.S. National Science Foundation under Award Number CMMI-1134953. The author wishes to acknowledge the sponsor. However, any opinions, findings, conclusions, and recommendations presented in this paper are those of the authors and do not necessarily reflect the views of the sponsors.

And finally, I would like to thank my family and friends for their moral support. 


\section{TABLE OF CONTENTS}

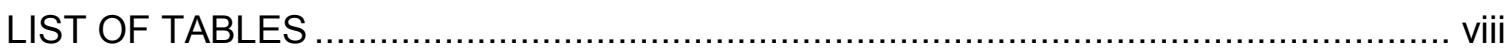

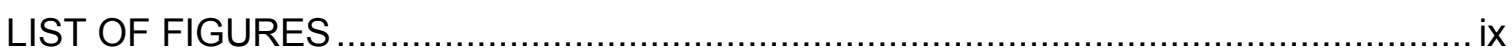

CHAPTER

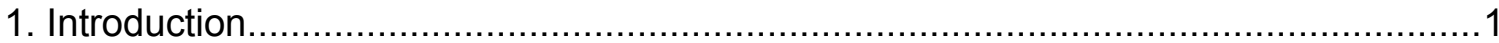

1.1 Background

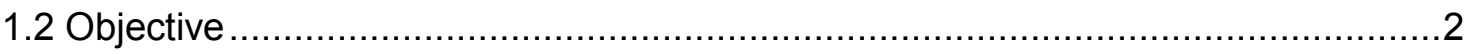

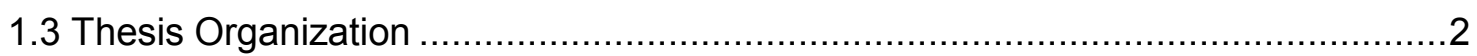

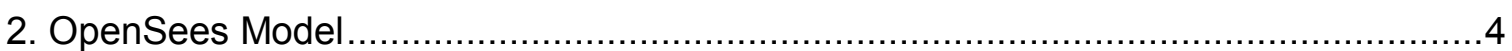

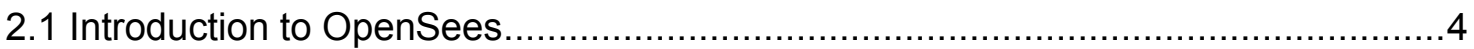

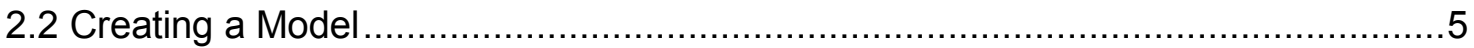

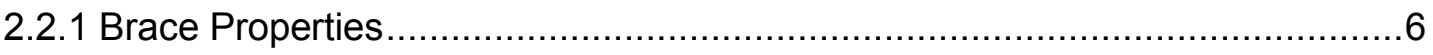

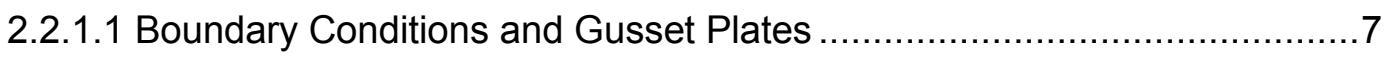

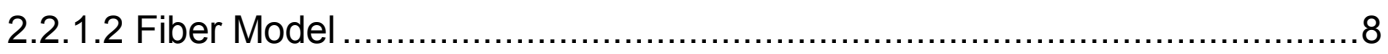

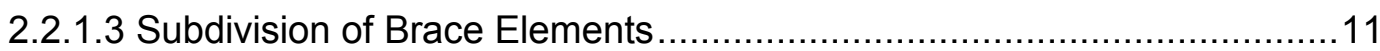

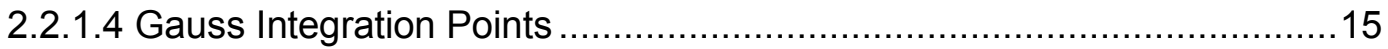

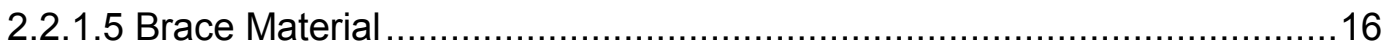

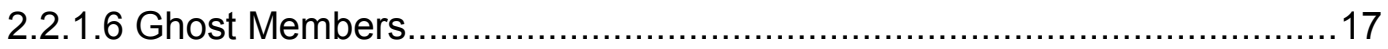

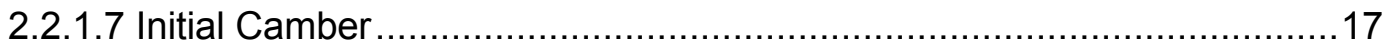

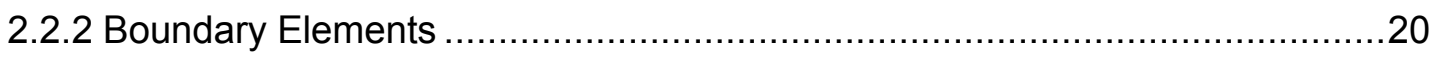

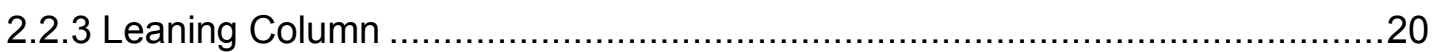

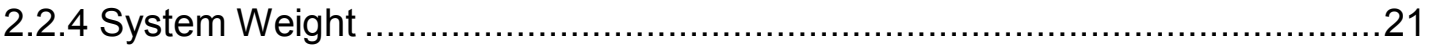

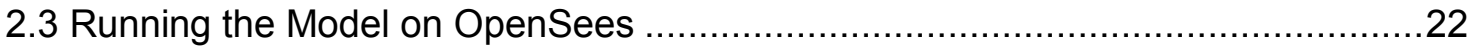

2.3.1 Choosing between OpenSees, OpenSeesSP, and OpenSeesMP ...............22

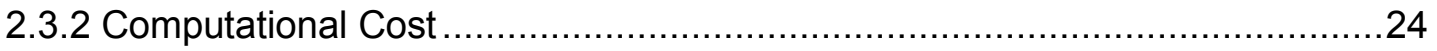

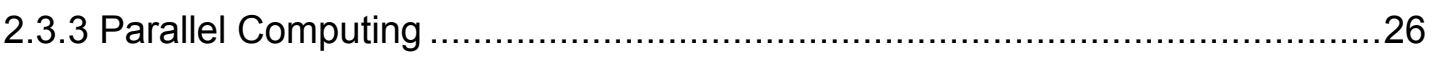

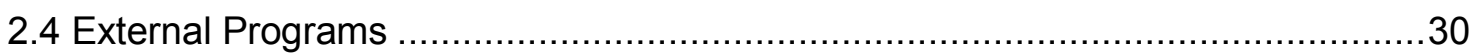

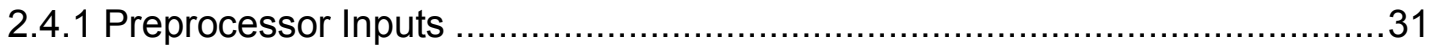

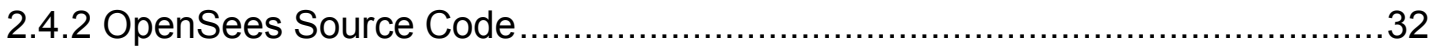

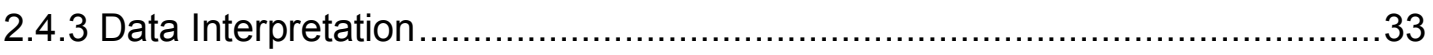

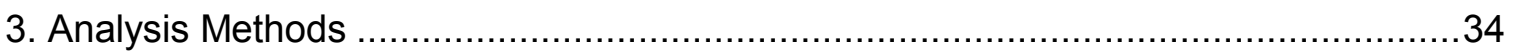

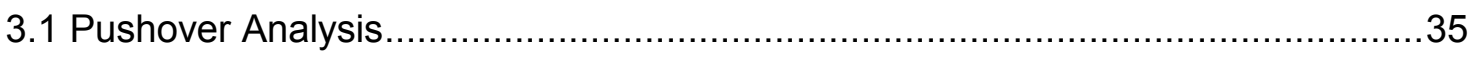

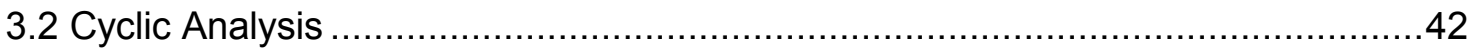

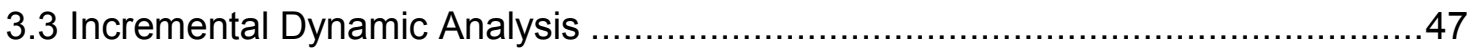

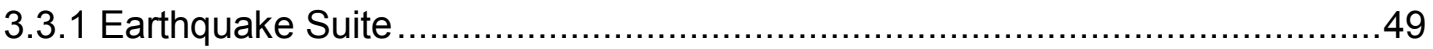




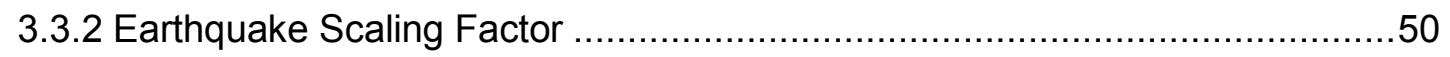

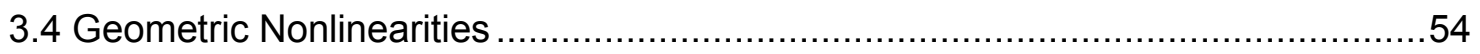

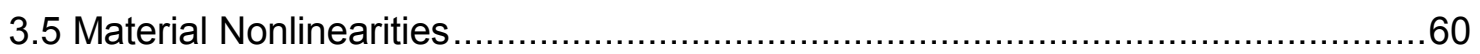

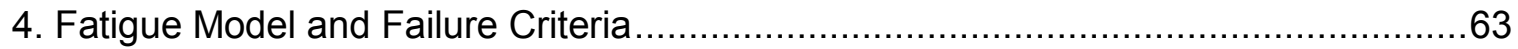

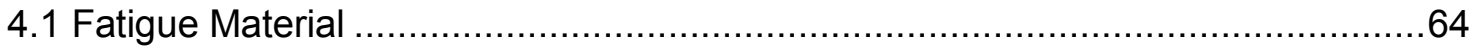

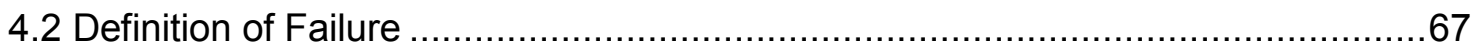

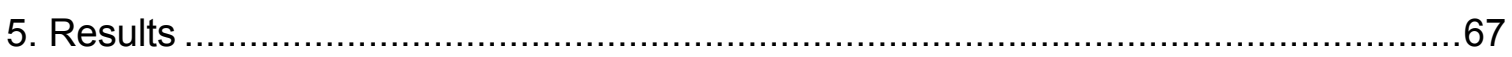

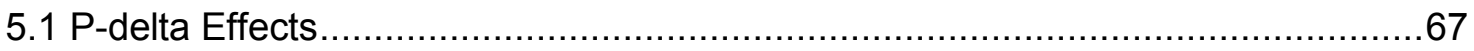

5.1.1 Monotonic Pushover ...................................................................... 68

5.1 .2 Incremental Dynamic Analysis ............................................................ 70

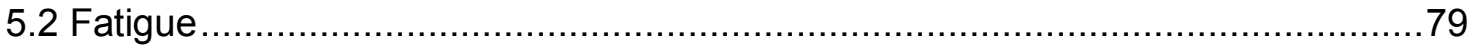

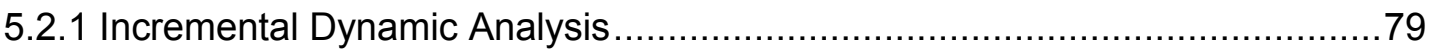

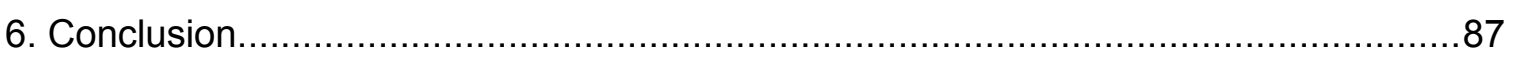

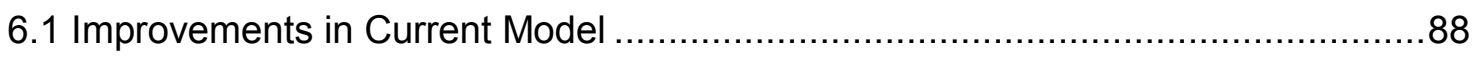

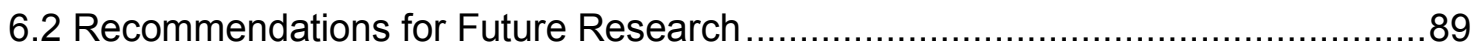

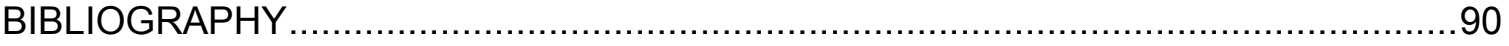
APPENDICES

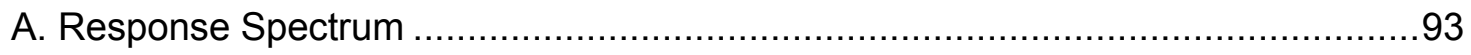

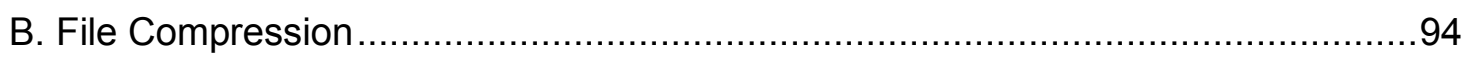

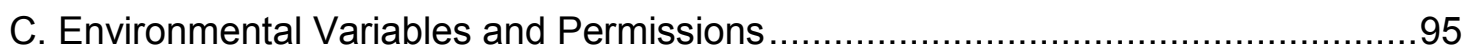

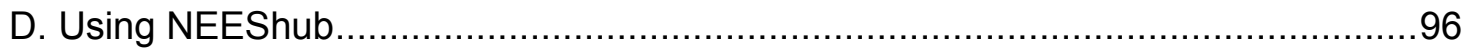

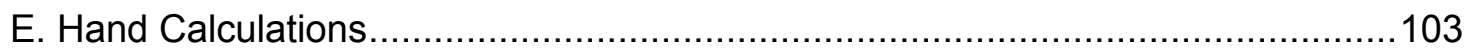

F. AISC Seismically Compact HSS Square Hollow Sections ..............................110 


\section{LIST OF TABLES}

Table

Page

Table 2-1: Seismically Compact HSS Braces chosen for analysis. ........................... 7

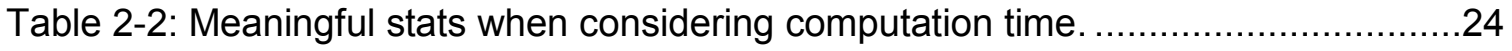

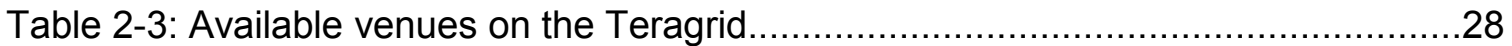

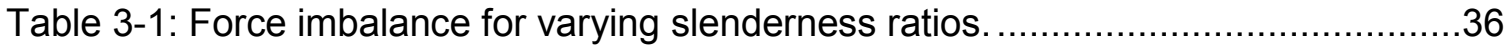

Table 3-2: Summary of displacement associated with first yield

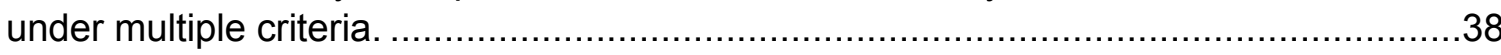

Table 3-3: SAC LA 2\% probability of exceedance in 50 years

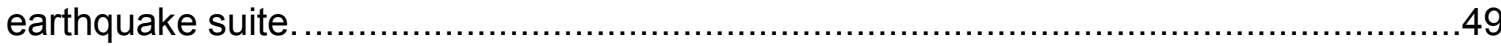

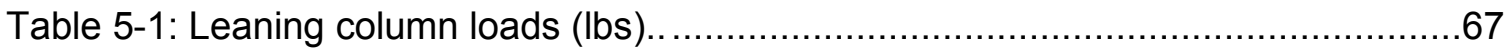

Table F-1: Seismically Compact HSS Square Hollow Sections ................................110 


\section{LIST OF FIGURES}

Figure Page

Figure 2.1: OpenSees Model (Not drawn to scale, drawn

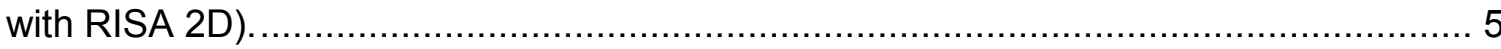

Figure 2.2: Variables to consider for defining braces

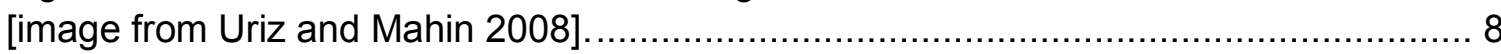

Figure 2.3: Effect of fiber placement within a cross-section

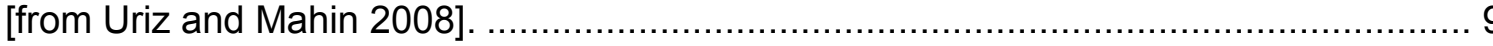

Figure 2.4: The effect of plane sections remaining plane [from Uriz and Mahin 2008].

Figure 2.5.a: The effects of slenderness on required subdivision

of the braces. Blue lines represent the subdivision without the consideration of fatigue and red lines represent the subdivision with fatigue. Results for Brace 1 under EQ2.

Figure 2.5.b: The effects of slenderness on required subdivision of the braces. Blue lines represent the subdivision without the consideration of fatigue and red lines represent the subdivision with fatigue. Results for Brace 2 under EQ2.

Figure 2.5.c: The effects of slenderness on required subdivision of the braces. Blue lines represent the subdivision without the consideration of fatigue and red lines represent the subdivision with fatigue. Results for Brace 3 under EQ2.

Figure 2.5.d: The effects of slenderness on required subdivision of the braces. Blue lines represent the subdivision without the consideration of fatigue and red lines represent the subdivision with fatigue. Results for Brace 4 under EQ2.

Figure 2.6: Effect of number of integration points

[Image from Uriz and Mahin 2008].

Figure 2.7.a: Effect of initial imperfection on Brace 1.

Figure 2.7.b: Effect of initial imperfection on Brace 2 ............................................ 18

Figure 2.7.c: Effect of initial imperfection on Brace 3...........................................19

Figure 2.7.d: Effect of initial imperfection on Brace 4............................................ 19

Figure 2.8: OpenSees run locally on an MSI GT60 2OKWS ....................................24

Figure 2.9: Flops/sec of the 500 best supercomputers in the

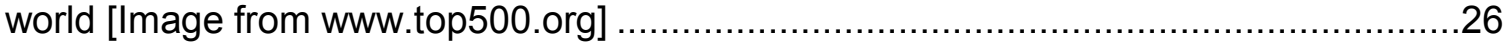

Figure 3.1: Eigen value check during pushover analysis. .......................................34

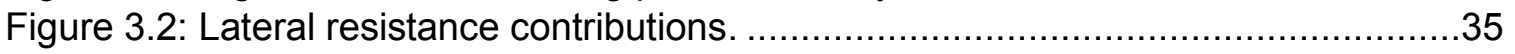

Figure 3.3: Base shear using pushover analysis................................................ 37

Figure 3.4.a: Base shear and axial load in Brace 1

under pushover analysis.

Figure 3.4.b: Base shear and axial load in Brace 2

under pushover analysis. 39 
Figure 3.4.c: Base shear and axial load in Brace 3

under pushover analysis.

Figure 3.4.d: Base shear and axial load in Brace 4

under pushover analysis. 41

Figure 3.5: Cyclic loading protocol for steel frames.

Figure 3.6.a: Hysteretic base shear and axial load

in Brace 1 under cyclic analysis.

Figure 3.6.b: Hysteretic base shear and axial load

in Brace 2 under cyclic analysis.

Figure 3.6.c: Hysteretic base shear and axial load

in Brace 3 under cyclic analysis.

Figure 3.6.d: Hysteretic base shear and axial load

in Brace 4 under cyclic analysis.

Figure 3.7: Initial scaling factor

Figure 3.8: Response for EQ 1. Where SF = a linear

increase \%SF = a percent increase.

Figure 3.9: Effect of structural resurrection on determination

of the R factor [from Hou and Qu 2014].

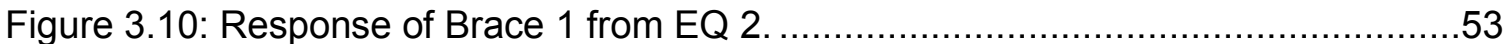

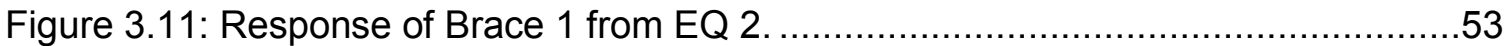

Figure 3.12: Directional assignments of forces in reference system. ..........................54

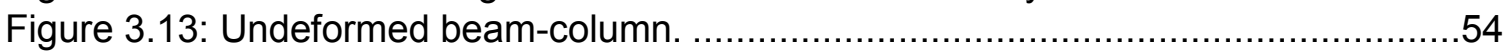

Figure 3.14: Deformed beam-column. ............................................................ 57

Figure 3.15: Simply supported beam under axial load

[from Denavit and Hajjar 2013].

Figure 3.16: Example hysteretic behavior with isotropic

hardening in tension and compression, respectively

[from McKenna and Fenves 2004].

Figure 3.17: Example accumulated damage and strain

of a model with arbitrary strain history, respectively

[Images from McKenna and Fenves 2004].

Figure 5.1.a: Leaning column effects on Brace 1 under pushover. ..............................68

Figure 5.1.b: Leaning column effects on Brace 2 under pushover. .............................68

Figure 5.1.c: Leaning column effects on Brace 3 under pushover..............................69

Figure 5.1.d: Leaning column effects on Brace 4 under pushover. .............................69

Figure 5.2.a: Leaning column effects on Brace 1

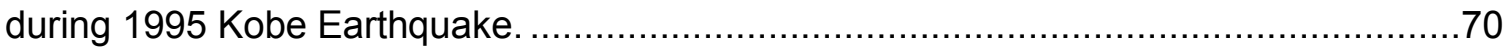

Figure 5.2.b: Leaning column effects on Brace 2

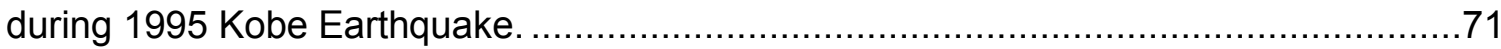

Figure 5.2.c: Leaning column effects on Brace 3

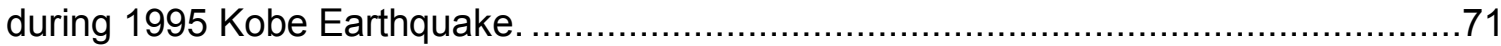

Figure 5.2.d: Leaning column effects on Brace 4

during 1995 Kobe Earthquake.

Figure 5.3.a: Median leaning column effects on Brace 1

under the EQ suite....

Figure 5.3.b: Median leaning column effects on Brace 2

under the EQ suite. 
Figure 5.3.c: Median leaning column effects on Brace 3

under the EQ suite

Figure 5.3.d: Median leaning column effects on Brace 4

under the EQ suite.

Figure 5.4.a: Normalized leaning column effects on Brace 1

under the EQ suite.

Figure 5.4.b: Normalized leaning column effects on Brace 2

under the EQ suite

Figure 5.4.c: Normalized leaning column effects on Brace 3

under the EQ suite.

Figure 5.4.d: Normalized leaning column effects on Brace 4

under the $E Q$ suite.

Figure 5.5.a: Fatigue consideration for Brace 1

under 1995 Kobe Earthquake.

Figure 5.5.b: Fatigue consideration for Brace 2

under 1995 Kobe Earthquake.

Figure 5.5.c: Fatigue consideration for Brace 3

under 1995 Kobe Earthquake.

Figure 5.5.d: Fatigue consideration for Brace 4

under 1995 Kobe Earthquake. .82

Figure 5.6.a: Fatigue consideration for Brace 1 under the EQ suite...........................83

Figure 5.6.b: Fatigue consideration for Brace 2 under the EQ suite............................83

Figure 5.6.c: Fatigue consideration for Brace 3 under the EQ suite...........................84

Figure 5.6.d: Fatigue consideration for Brace 4 under the EQ suite............................84

Figure 5.7: Normalized representation of the mean effects of fatigue. .........................85

Figure A.1: MCE response spectra, image from Sanchez-Zamora 2013. ...................93

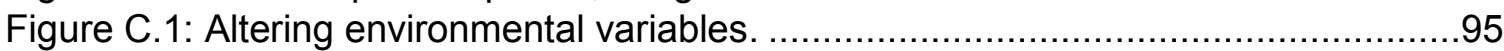

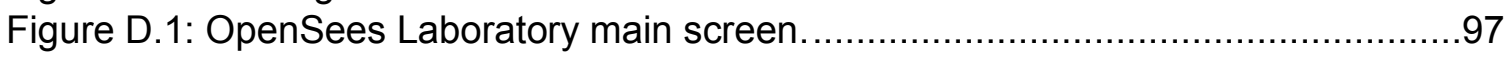

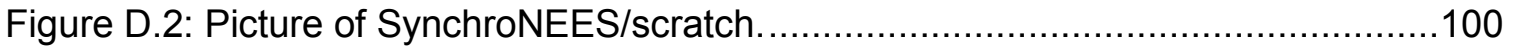




\section{Introduction}

\subsection{Background}

Concentrically Braced Frames (CBF) are a class of lateral force resisting system that provide high strength and stiffness. CBFs are typically simpler, more economical, and have lower interstory drifts than other lateral force resisting systems used in practice. For these reasons, the percent of new CBFs in commercial construction has been on the increase [Ferch 2004; Uriz and Mahin 2008]. However, a more stiff system leads to higher accelerations and low drift capacities, non-ideal for seismic response. But with proportional member sizing and detailing CBFs can perform well in high seismicity areas [Sabelli et al. 2013].

Performance of a CBF is largely dependent on the performance of its braces. Under substantial lateral loading the braces in the CBF will typically form plastic hinges that experience large rotational demands and therefore have large strain histories [Uriz and Mahin 2008; Jain and Goel 1978; Lee and Goel 1987; Shaback and Brown 2003; Stojadinović 2003; Tremblay et al. 2003]. The large strain histories can cause rupture in the section, this phenomena is known as fatigue. High-cycle fatigue and low-cycle fatigue is the accumulation of plastic strains over many events and within a single event, respectively. Fatigue life should be considered during analytical studies to improve the accuracy of plastic behavior. Many papers have stated the importance of considering fatigue life, in particular low-cycle fatigue, during strong seismic events when determining displacements and structural integrity [Uriz and Mahin 2008; Ikeda and Mahin 1986; Li and Fahnestock 2013; Li and Fahnestock 2013]. 


\subsection{Objective}

The main purpose of this thesis is to develop a further understanding of the effects slenderness ratio has on low-cycle fatigue during seismic excitation. The brace slenderness ratios analyzed are $300,200,100$, and 50 for seismically compact HSS square tube sections. These slenderness ratios represent the tension only brace limit, Special Concentrically Braced Frames (SCBF) limit, Ordinary Concentrically Braced Frame (OCBF) limit, and the compression only brace limit, respectively [AISC 2005]. A full spectral response ( 0.1 to 2.0 second) of each brace is conducted using Incremental Dynamic Analysis (IDA) under an SAC Joint Venture earthquake suite for $2 \%$ probability of exceedance in 50 years in the Los Angeles area. [SAC 2011]

Results of computational simulation are compared using their Seismic Response Modification Factor ( $\mathrm{R}$ factor). Along with fatigue several other parameter studies are conducted in this thesis to further develop understandings on the effects of slenderness. This thesis also analyzes methods to improve computational efficiency. Note that Buckling Restrained Braced Frames (BRBF), and the performance of other lateral force resisting systems, are out of the scope of this thesis and will not be discussed.

\subsection{Thesis Organization}

This thesis contains 6 chapters, topics covered are as follows:

- Chapter 2 covers the two-dimensional model used throughout analysis and its respective properties. The model is created and ran with OpenSees, discussions on how to run OpenSees and supporting programs are also presented in this chapter. 
- Chapter 3 presents the three methods of analysis used in this thesis: pushover, cyclic, and transient during incremental dynamic analysis. It also discusses the effects of geometric and material nonlinearities on the model.

- Chapter 4 briefly discusses the use of the pre-coded fatigue material and the definition of failure in the model.

- Chapter 5 presents and discusses the effects that slenderness has on leaning columns and fatigue life, the two main parameter studies conducted.

- Chapter 6 presents recommendations for improving the current model and recommendations for future research. 


\section{OpenSees Model}

This chapter discusses the 2-D analytical model that is used throughout analysis. This chapter introduces the program in which the model is created and analyzed. All elements within the model are described in full detail in this chapter including member properties, boundary conditions, and loading conditions. The latter portion of this chapter discusses how to efficiently run analysis and introduces some of the external programs needed to assist analysis.

\subsection{Introduction to OpenSees}

OpenSees is a free open source software developed to assess structural and geotechnical performance of a system subjected to many different loading cases.

OpenSees is frequently used in academia due to its ability to easily be modified and its ability to perform nonlinear analysis. It is also under constant development by academic research sponsored by the Pacific Earthquake Engineering Research Center (PEER). [McKenna and Fenves 2004]

OpenSees is used in this thesis due to its vast library of pre-coded materials and solvers, in particular its nonlinear solvers including a fatigue damage counter. Many of the pre-coded materials have extensive analytical and experimental research to hold them to precise calibration, therefore results of analysis can be stated with a slightly higher level of confidence. The accessible source code allows for case study investigations as to how the solvers work. Again since this program has had so much research conducted with it, there are several papers that can verify results of the case studies for the solvers. Finally this thesis is a parameter study on the effects of brace slenderness, script based programs are typically more efficient than Graphical User Interface (GUI) based programs for parameter studies. [McKenna and Fenves 2004] 


\subsection{Creating a Model}

The model used throughout analysis is a two dimensional three degree of freedom framework using OpenSees' basic model builder. The model consists of a single concentrically braced frame (CBF) with a typical bay width of 30' and story height of 13', with a single leaning column offset 5 ' to the left. Figure 2.1, shown below, shows a simplified view of the model. Note that system weight is lumped at the beam-column interface, the system neglects self-weight, the system has a typical $5 \%$ damping, the vertical load applies a P- $\Delta$ effect of leaning columns, and the horizontal load represents the equivalent lateral load/displacement for pushover, cyclic, and transient excitations. All elements in this model are created using object oriented coding for efficiency. The next sections of this chapter further describe cross-sections, material selection, and boundary conditions used for each element.

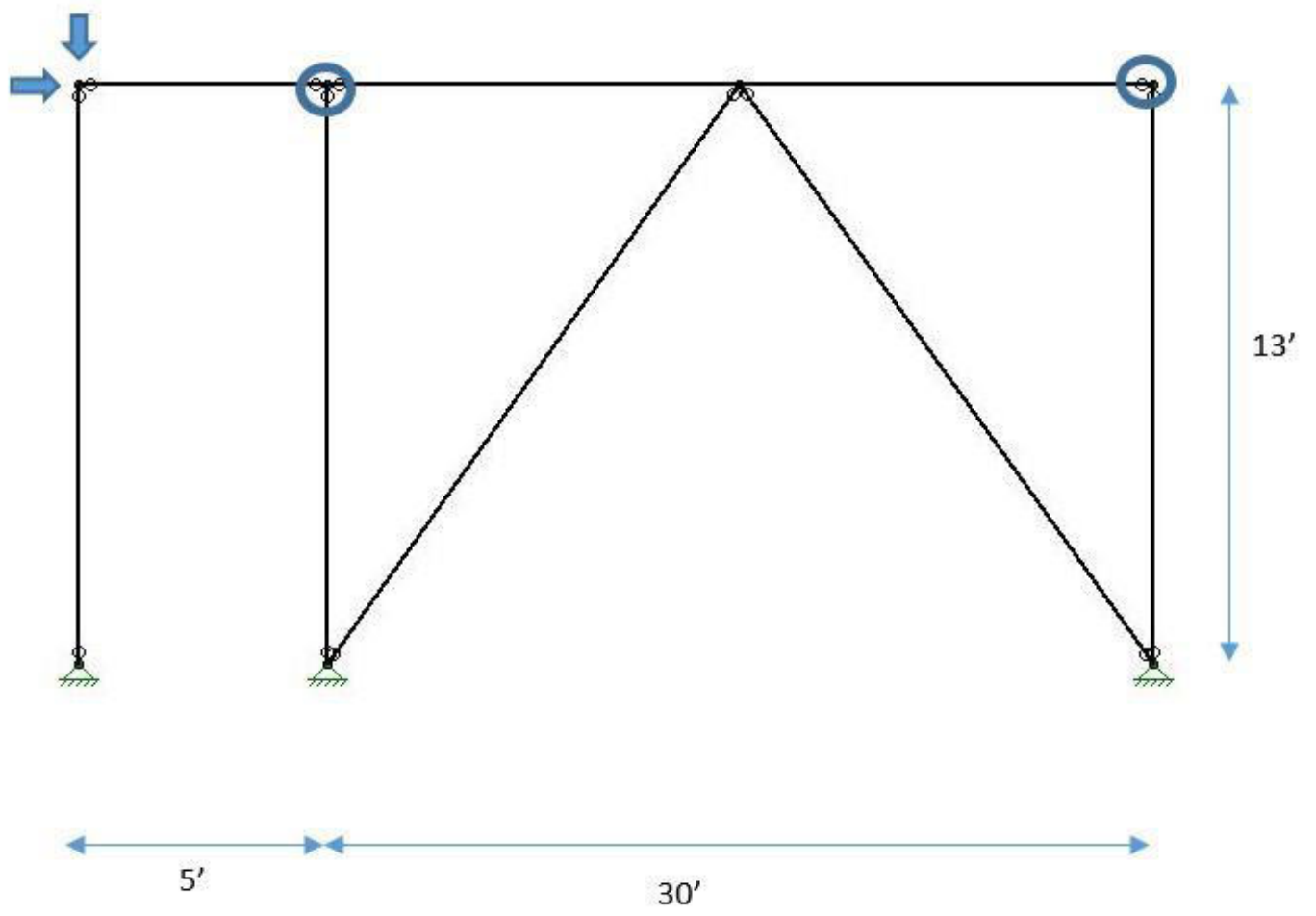

Figure 2.1: OpenSees Model (Not drawn to scale, drawn with RISA 2D). 


\subsubsection{Brace Properties}

Brace selection is a very important step, as the purpose of this thesis is to analyze the effects that brace slenderness has on low-cycle rupture fatigue. Predetermined parameters at the start of the project set the slenderness values to $50,100,200$, and 300 and that seismically compact HSS square tube sections be used. Since AISC Steel Construction Manual does not currently have fabricated HSS square braces that have the exact slenderness values while being seismically compact it was decided to use custom sized braces. Appendix F contains the full list of seismically compact members available in the manual [AISC 2011]. Below is the derivation of the equation used to determine the brace sizing for a given slenderness.

$$
\begin{gathered}
\frac{k L}{r}=\frac{1.0 L_{D}}{r}=\frac{L_{D}}{r} \\
r=\sqrt{\frac{I}{A}}=\sqrt{\frac{\frac{1}{12}\left[B^{4}-(B-2 t)^{4}\right]}{B^{2}-(B-2 t)^{2}}} \\
=\frac{B}{\sqrt{12}} \sqrt{\frac{1-\left(1-2 \frac{t}{B}\right)^{4}}{1-\left(1-2 \frac{t}{B}\right)^{2}}} \\
=\frac{B}{\sqrt{12}} \sqrt{1+\left(1-2 \frac{t}{B}\right)^{2}} \\
\left(\frac{k L}{r}\right)=\frac{L_{D}}{r}=\frac{\sqrt{12} L_{D}}{B} \frac{1}{\sqrt{1+\left(1-2 \frac{t}{B}\right)^{2}}}
\end{gathered}
$$

With Equation 1, brace sizing was determined by trying nominal values for brace width, $B$, which was used to calculate thickness, $t$, and then checked to ensure the brace was seismically compact. The braces are made of ASTM A500 Gr. B steel, typical for HSS 
square sections, which has a minimum yield stress of $46 \mathrm{ksi}\left(\mathrm{F}_{\mathrm{y}}\right)$ and a Young's Modulus of $29,000 \mathrm{ksi}(\mathrm{E})$. Further discussion of brace material properties will be covered in later sections. The check for seismic compactness is done using AISC Seismic Code Provisions, shown with Equation 2 below. [AISC 2005]

$$
\frac{B-2 t}{t} \leq 0.64 \sqrt{\frac{E}{F_{y}}}=0.64 \sqrt{\frac{29000}{46}}=16.07
$$

The table below shows the properties of the braces used throughout analysis of this thesis. Supporting hand calculation are located in Appendix E.

Table 2-1: Seismically Compact HSS Braces chosen for analysis.

\begin{tabular}{|l|c|c|c|c|}
\hline Section & $\mathbf{k l / r}$ & $\mathbf{B}$ & $\mathbf{t}$ & $\mathbf{b} / \mathbf{t}$ \\
\hline Brace 1 & 50 & 12.5 & 0.86255 & 14.49187 \\
\hline Brace 2 & 100 & 6.5 & 0.70849 & 9.17444 \\
\hline Brace 3 & 200 & 3.5 & 0.65778 & 5.32092 \\
\hline Brace 4 & 300 & 2.5 & 0.67658 & 3.69504 \\
\hline
\end{tabular}

\subsubsection{Boundary Conditions and Gusset Plates}

Braces are modeled as 2-D trusses (e.g. all braces have pin-pin boundary conditions) to simplify analysis and to ensure all of the ductility is coming from the braces. Gusset plates were not included in analysis as they would absorb energy and create extra ductility in the system. Further analysis should be done to see the effect that gusset plates have on low-cycle fatigue, but this is outside of the scope of this thesis. Refer to [Uriz and Mahin 2008] for more information on the effects of gusset plates on hysteretic response using an energy approach. 


\subsubsection{Fiber Model}

Braces used in this model are based on a force formulation of a fiber cross section. A force formulation approach offers several advantages over the more common displacement formulation: "(a) the force-interpolation functions are always exact in the absence of second-order effects; (b) a single element can be used to represent the curvature distribution along the entire member with sufficient accuracy through selection of a sufficient number of integration points (monitoring sections); and (c) the formulation has proven numerically robust and reliable, even in the presence of strength softening, as is the case for buckling steel braces." [Uriz and Mahin 2008; Spacone et al. 1996] A fiber model is used over other FEA models because the fiber model can analyze damage in each fiber, and the remove itself from the model at failure which is critical to capture the low-cycle fatigue. The fiber model can also mimic realistic material properties with the inclusion of residual stresses due to fabrication and construction. [Uriz and Mahin 2008]

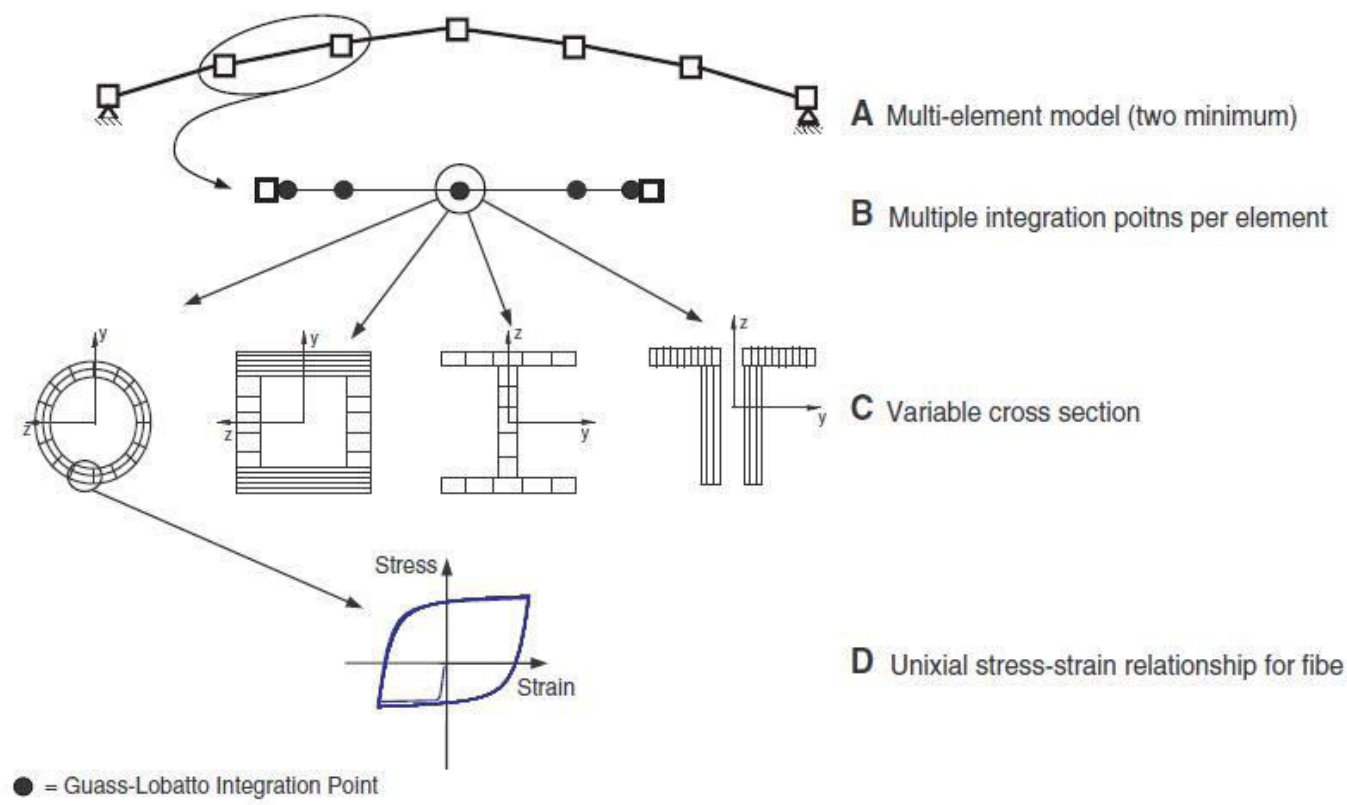

Figure 2.2: Variables to consider for defining braces [image from Uriz and Mahin 2008]. 
Figure 2.2 above represents the variables that need to be defined when creating the fiber model for the brace element. Image A shows the brace broken into subdivisions, discussed in detail in Section 2.2.1.3. Image B shows the number of Guass-Lobatto integration points within each subdivision, discussed further in Section 2.2.1.4. Image C shows some possible fiber cross section, but square HSS tubing has already been decided on as stated in earlier sections. Image D shows the stress-strain relationship, discussed in detail in Section 2.2.1.5.

The number of fibers and their respective location within the fiber cross section effects how well the nonlinear element behaves. Braces used in this thesis use a 5 by 4 fiber patch for the top and bottom face and a 2 by 5 fiber patch for the side walls, where numbers represent patches y by $z$ as shown in Figure 2.2 (Image C). Figure 2.3 below show that fibers around the perimeter have more effect than fibers across thickness.

\begin{tabular}{ccc}
\hline ID & $\begin{array}{c}\text { Number of fibers } \\
\text { across thickness }\end{array}$ & $\begin{array}{c}\text { Number of Fibers } \\
\text { around the perimeter }\end{array}$ \\
\hline \hline A & 1 & 5 \\
B & 1 & 10 \\
C & 5 & 10 \\
D & 10 & 60 \\
E & 2 & 30 \\
\hline
\end{tabular}

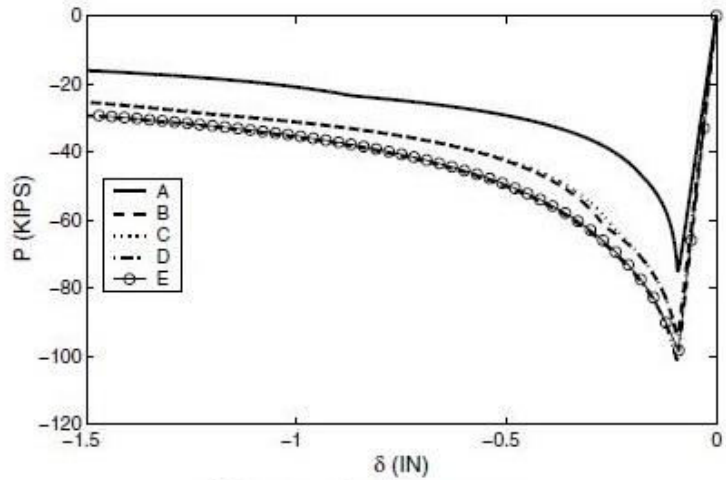

(a) monotonic response

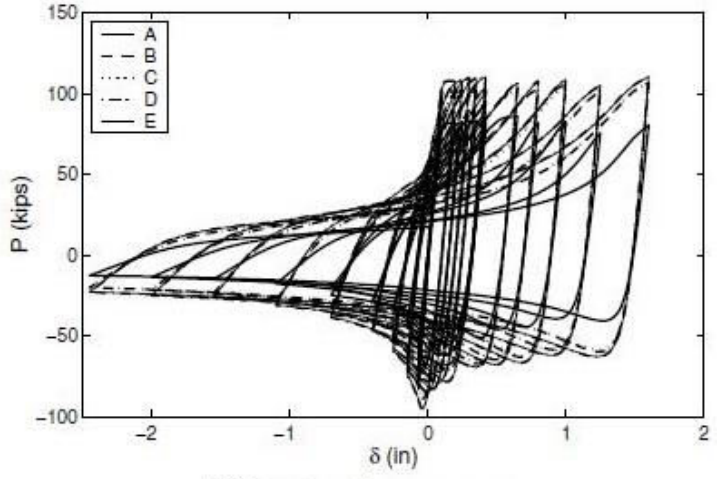

(b) hysteretic response

Figure 2.3: Effect of fiber placement within a cross-section [from Uriz and Mahin 2008]. 
The fiber model defined above has several assumptions and limitations:

- The model follows small deformation theory. Therefore no rigid body modes are considered.

- Nonlinear geometry under large displacements are accounted for with corotational transformations. [Filippou and Fenver 2004]

- Shearing deformations are ignored.

- Cross sections do not distort under loading. Therefore plane sections remain plane.

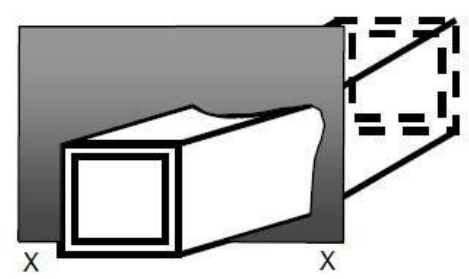

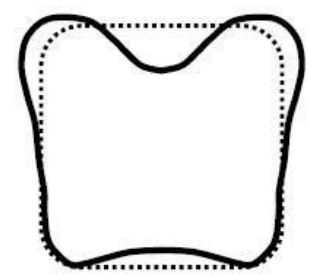

Section $X-X$

Figure 2.4: The effect of plane sections remaining plane [from Uriz and Mahin 2008].

- Since sections are compact it is assumed that no local buckling will occur.

- Elements are divided into a sufficient number of sub-elements with a sufficient number integration points to accurately represent local deformations and strains.

- Multi-axial stress states are ignored. This assumption has a drastic effect on the brace-gusset plate interface but has less effect here since the system is modeled as pinned boundary conditions.

- Torsional response is neglected, therefore lateral torsional buckling modes are not considered.

- Initial residual stresses due to fabrication and construction are disregarded.

For a more detailed explanation on these assumptions and limitations refer to [Uriz and Mahin 2008]. 


\subsubsection{Subdivision of Brace Elements}

Braces need to be broken up into sufficient number of sub-elements in order to accurately represent local deformations and strains at critical sections of the brace. This is essential to formulate the effects of low-cycle fatigue. It is recommended to use at least two subdivisions to capture the effect of global force-displacement behavior, but strains will not be accurately modeled with just two subdivisions. Since the member strains are crucial for fatigue accumulation it is recommended that a minimum of twenty sub-elements be used. [Uriz and Mahin 2008]

A parameter study was conducted evaluating the effect of subdividing the braces for when fatigue is and is not considered. Results can be seen in Figure 2.5.a to 2.5.d below.

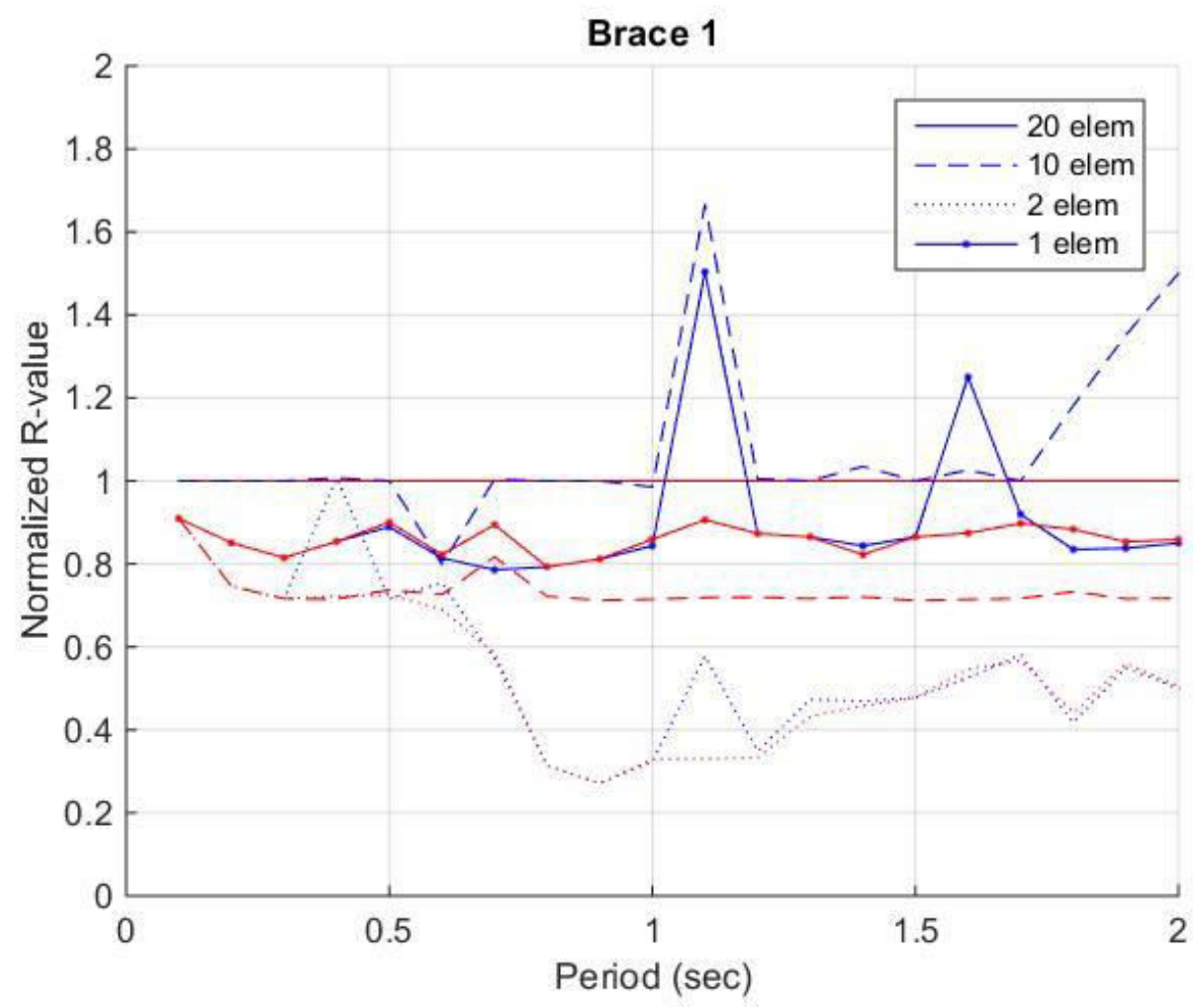

Figure 2.5.a: The effects of slenderness on required subdivision of the braces. Blue lines represent the subdivision without the consideration of fatigue and red lines represent the subdivision with fatigue. Results for Brace 1 under EQ2. 


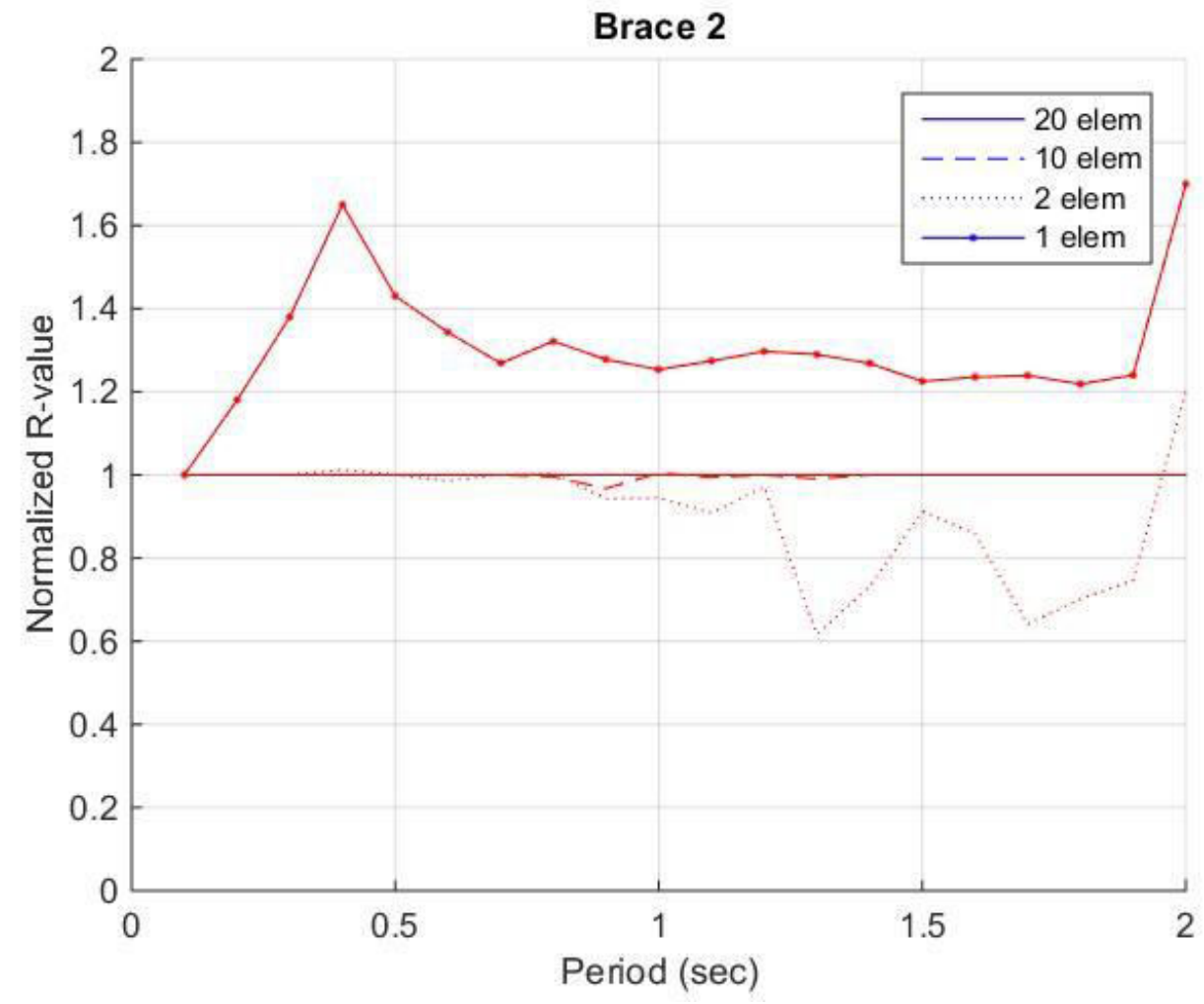

Figure 2.5.b: The effects of slenderness on required subdivision of the braces. Blue lines represent the subdivision without the consideration of fatigue and red lines represent the subdivision with fatigue. Results for Brace 2 under EQ2. 


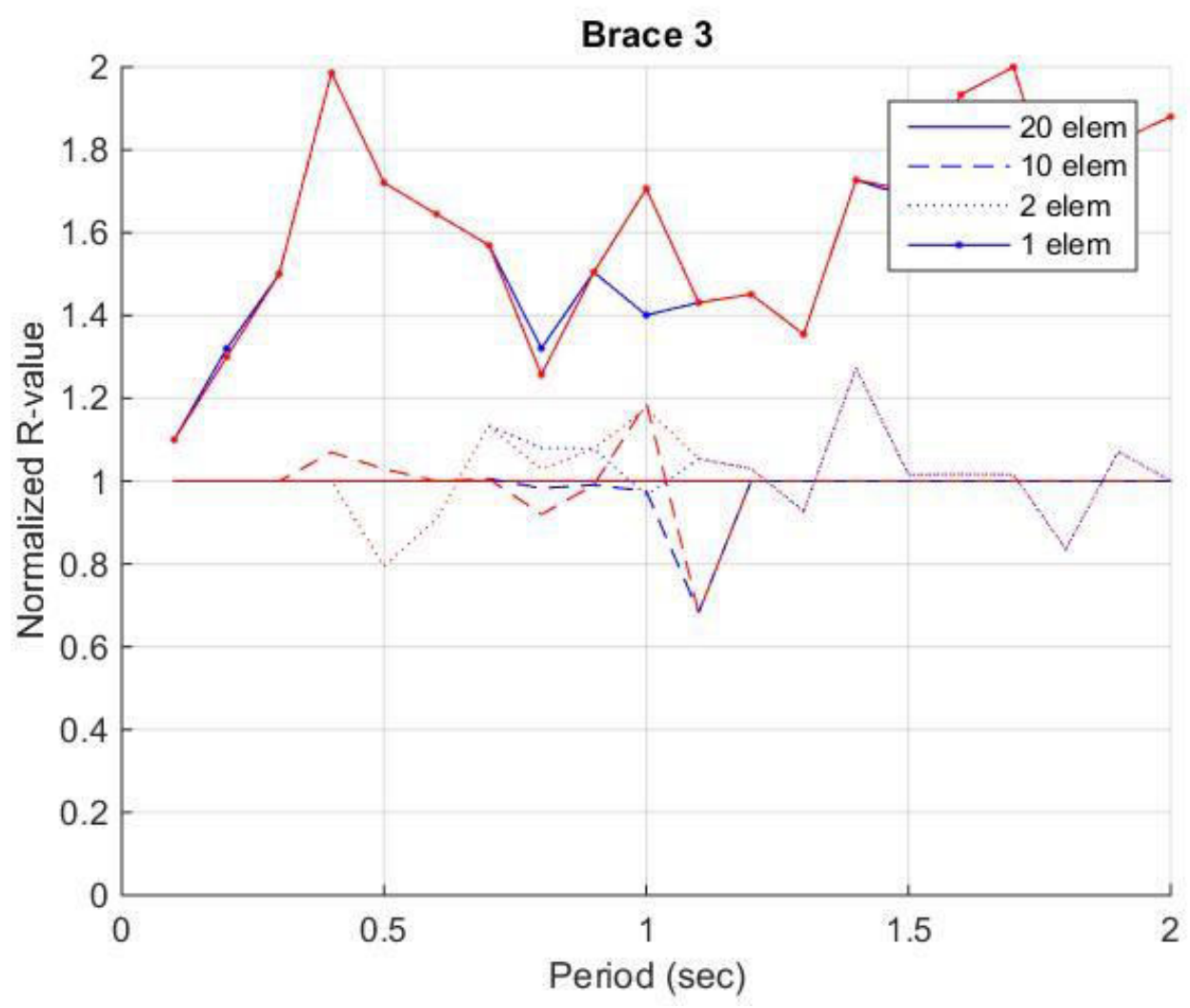

Figure 2.5.c: The effects of slenderness on required subdivision of the braces. Blue lines represent the subdivision without the consideration of fatigue and red lines represent the subdivision with fatigue. Results for Brace 3 under EQ2. 


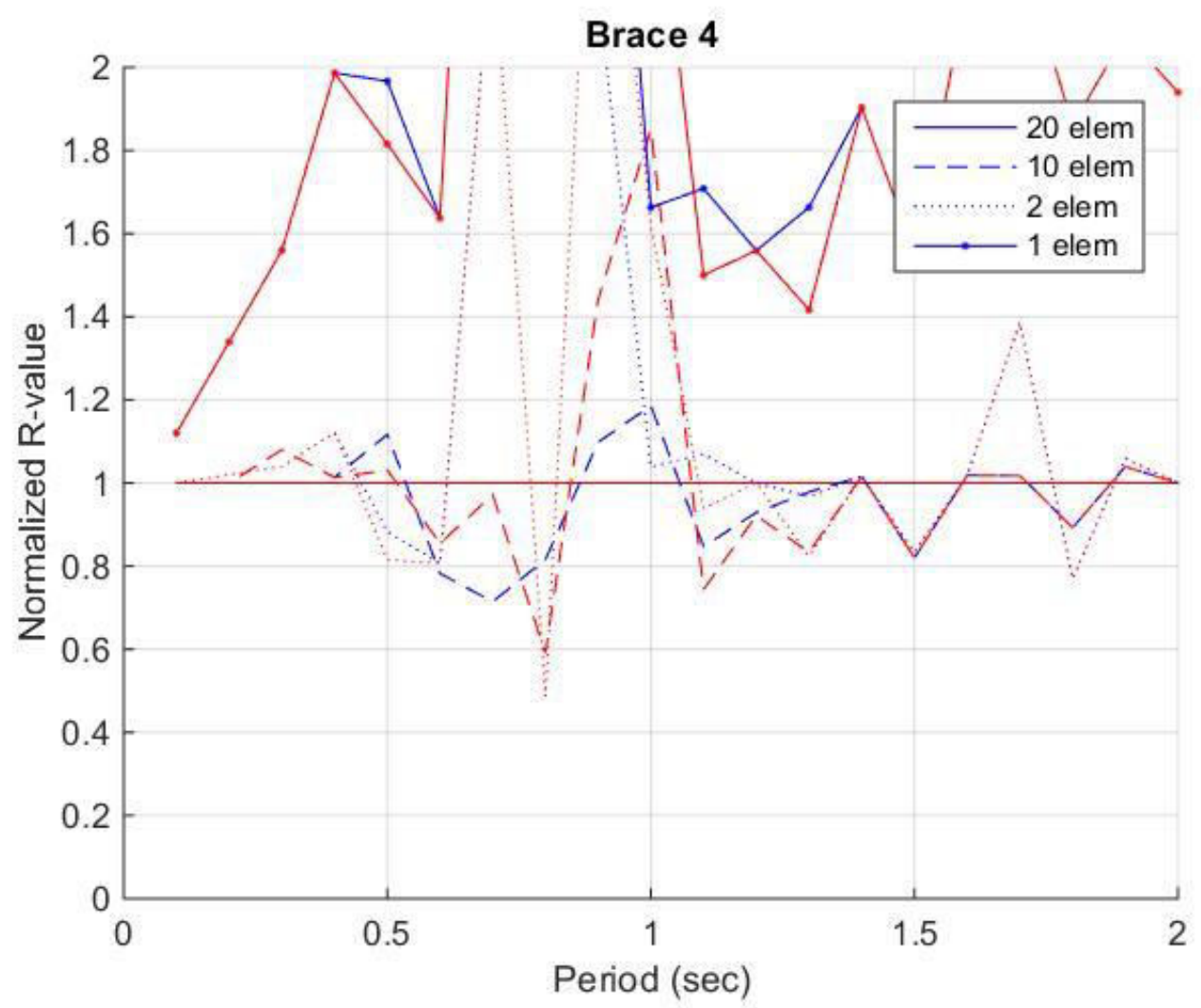

Figure 2.5.d: The effects of slenderness on required subdivision of the braces. Blue lines represent the subdivision without the consideration of fatigue and red lines represent the subdivision with fatigue. Results for Brace 4 under EQ2.

Brace 2 seems to be the least sensitive to the subdivision of the brace while Brace 4 seems to be the most sensitive. A 20 elements per brace subdivision, the recommended minimum default, seems sufficient for analysis of Brace 2 but may not be enough for accurate results of the other braces. Unfortunately this study was ran in the latter portion of this thesis, so the default of 20 subdivisions was used throughout analysis. Refer to Chapter 5 for further discussion on how this could have affected the final results. In this study no clear trends of the effects of fatigue can be correlated due to the large scatter in the data. 


\subsubsection{Gauss Integration Points}

Due to a large amount of research on the effects on the quantity of Gaussian integration points in the quadrature, no addition parameter study was conducted for this thesis [Neuenhofer and Filippou 1998, Uriz and Mahin 2008, Neuenhofer and Filippou 1997]. Refer to Figure 2.2 (Image B) for a visual representation of the number of Gaussian integration points per sub-element. Figure 2.6.a and 2.6.b below show the effect that the number of Gauss points has on monotonic and hysteretic response, respectively. From these figures it can be seen that three points are sufficient for analyzing post-buckling behavior. Two point integration results in an under-integration in the Gaussian quadrature and therefore shouldn't be used in post-buckling analysis. [Uriz and Mahin 2008]

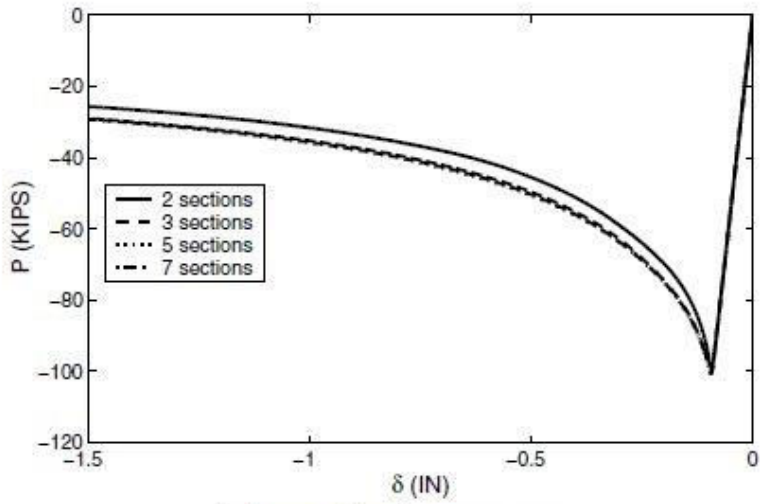

(a) monotonic response

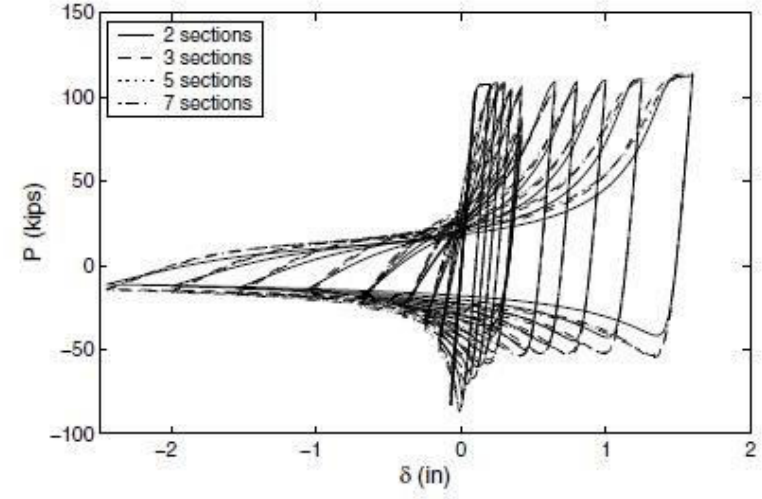

(b) hysteretic response

Figure 2.6: Effect of number of integration points [Image from Uriz and Mahin 2008]. 


\subsubsection{Brace Material}

The braces on the frame are modeled with two different types of materials depending if fatigue is under consideration. Without fatigue the brace material OpenSees uses is uniaxial Giuffré-Menegotto-Pinto steel with isotropic strain hardening (Steel02). The braces are HSS Sections with A500 Gr.B Steel $\left(F_{y}=46 k s i\right)$ [AISC 2011] with a strain hardening ratio of 0.01 . Default of values for isotropic hardening and other parameters are used based on recommendations in the OpenSees manual along with several papers [Filippou and Fenves 2004; Uriz and Mahin 2008; McKenna and Fenves 2004]. Refer to Section 3.5 for discussion of Steel02 material nonlinearities.

With fatigue under consideration the brace continues to use the uniaxial GiuffréMenegotto-Pinto steel as the "parent" material, but now a secondary fatigue material that does not affect any of the properties of the brace is included in the model. The fatigue material was built specifically to capture the effects of low-cycle fatigue using a modified rainflow cycle counting algorithm known as Miner's Rule. Miner's Rule, based off a Coffin-Manson log-log relationship, uses a linear strain accumulation model to determine failure due to fatigue [McKenna and Fenves 2004; Uriz and Mahin 2008]. Meaning that once the strain in a given element surpasses a set value, damage will accumulate. When the sum of the damage reaches $100 \%$ of the capacity, the Young's modulus of the parent material is set to zero for the element. See Figure 3.17 for a visual representation of fatigue accumulation.

Default values for material strings have been calibrated from Ballio and Castiglioni 1995 and Uriz and Mahin 2008. Further discussion of the fatigue model can be found in Section 4.1. Refer to Section 3.5 for discussion of fatigue material nonlinearities. 


\subsubsection{Ghost Members}

Ghost members are perfectly straight members overlain on the braces to ensure computational stability after the braces fail. These braces only have an area of 0.05 square inches, so any lateral stiffness these braces supply is negligible. Before these 'ghost members' were implemented, solvers would often fail or have singularity, resulting in OpenSees crashing.

\subsubsection{Initial Camber}

Computer models idealize member as perfectly straight and free of imperfections, therefore members will never buckle under pure axial loading. However all members in reality will have some inherent camber and local imperfections due to the fabrication and construction process. To force members to buckle within the simulation an initial camber is added to the center of each brace. A parameter study was run to view the effects camber had on post-buckling behavior for each of the slenderness ratios. Figure 2.7 on the next pages show the results of the study for pushover analysis.

An initial camber of $0.1 \%$ of the brace length was chosen based on the data presented in the figure. Initial cambers larger than $0.1 \%$ tend to reduce the effects of strain hardening and softening, and initial cambers much larger than $0.1 \%$ greatly reducing the overall lateral capacity of the system. Figure 2.7 also shows that stockier braces are more sensitive to the amount of initial camber in the system. This makes sense because with stockier braces the compression brace have greater percent contribution to lateral stiffness than slender braces, therefore more sensitive to reduction in the axial capacity of the compression brace due to induced moments. Results of the parameter study are supported in Uriz and Mahin 2008. Which states that an initial camber of 0.05 to $0.1 \%$ of the brace length is recommended at the brace midspan. 


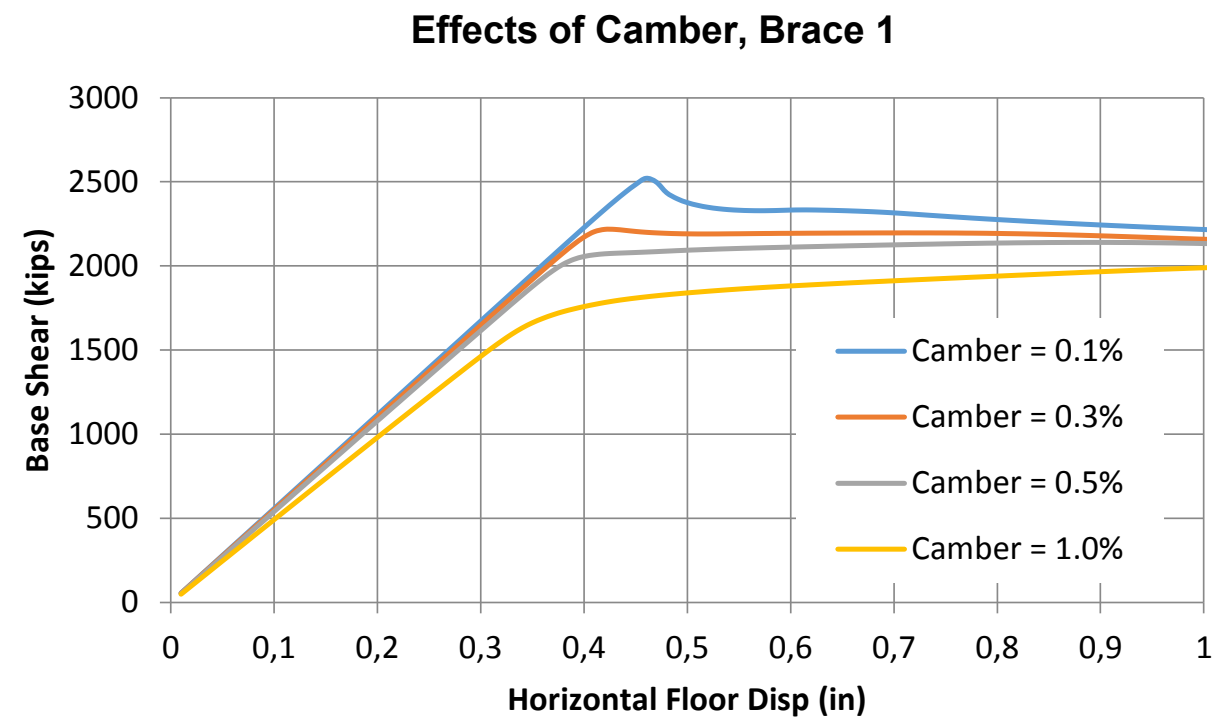

Figure 2.7.a: Effect of initial imperfection on Brace 1.

\section{Effects of Camber, Brace 2}

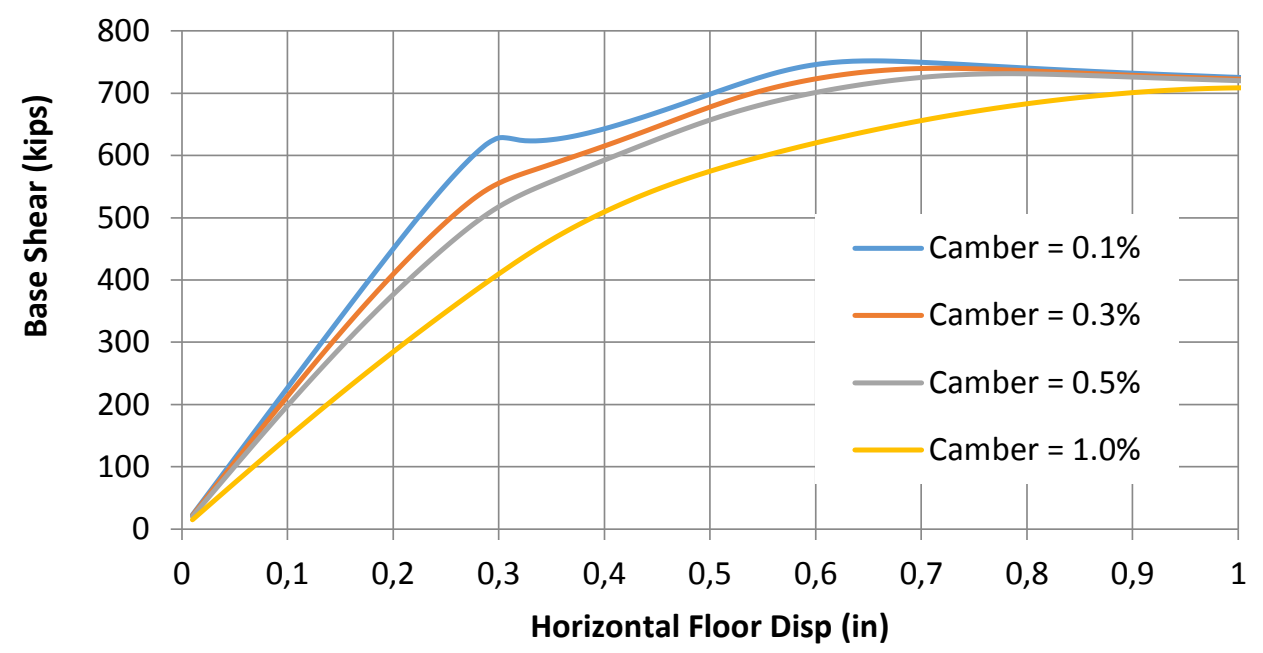

Figure 2.7.b: Effect of initial imperfection on Brace 2. 


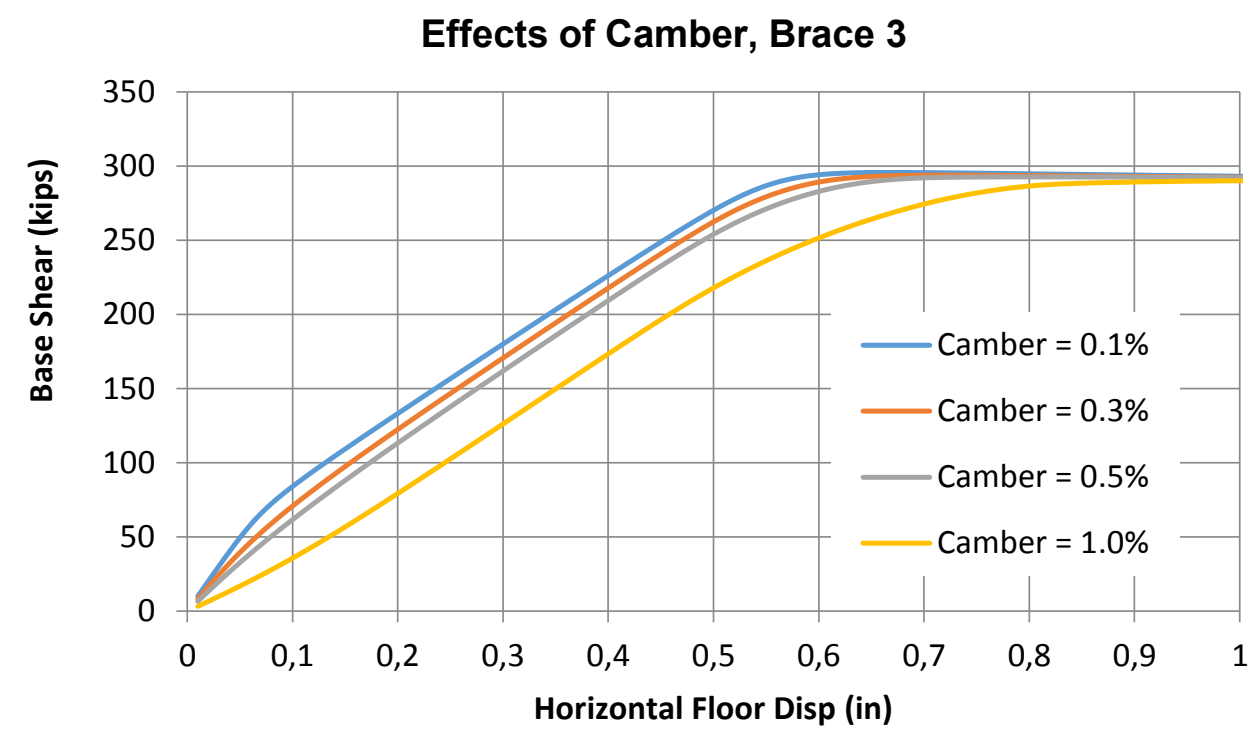

Figure 2.7.c: Effect of initial imperfection on Brace 3.

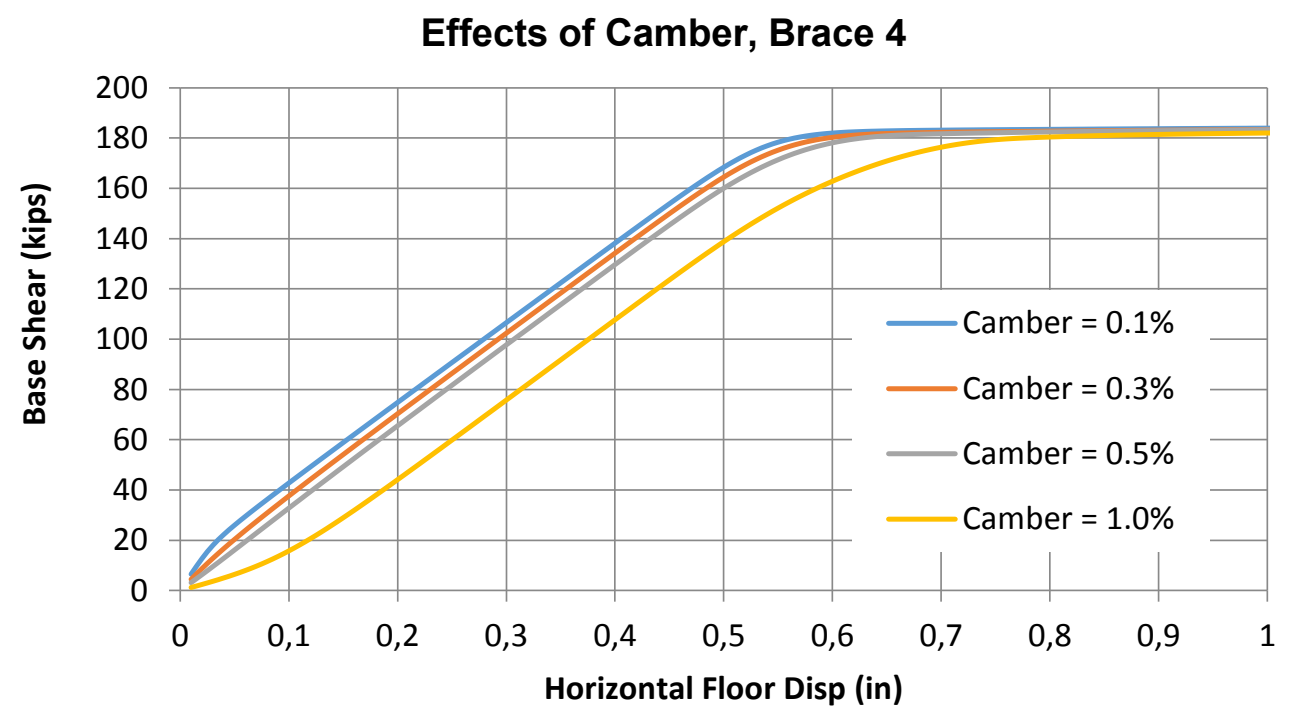

Figure 2.7.d: Effect of initial imperfection on Brace 4. 


\subsubsection{Boundary Elements}

All boundary elements are intended to act as ideally rigid in this model. The purpose of making the boundary members ideally rigid is to reduce the effects of displacements and energy dissipation caused by boundary elements. Boundary elements should not exhibit any yielding and the beam should not deflect noticeably at midpoint, non-rigid deflections may help the braces experiencing axial shortening/elongation. To achieve this, elements were designed to be elastic beam columns made of uniaxial Giuffre-Menegotto-Pinto steel material with a Young's Modulus, cross-sectional area, and second area moment of $29,000 \mathrm{ksi}, 100,000$ square inches, and 100,000 inches to the fourth, respectively. Refer to Section 2.2.1.5 for further discussion of Steel02.

\subsubsection{Leaning Column}

The purpose of running analysis on a simplified frame is to gain insight on how real structures are going to behave under real loading. The leaning gravity column is added to represent the presence of multiple gravity frames that the CBF has to support with lateral resistance. To capture the leaning column effect a study was done to capture how the various braces performed against multiple leaning loads. Refer to Section 5.1 for results conducted in pushover and transient analysis.

The leaning effect is created with a vertical load applied to the gravity frame offset to the left of the CBF bay. The magnitude of the load is calculated using Equation 3 below.

$$
P_{\text {leaning }}=\Delta_{\text {drift }} h K
$$

Where $\Delta_{\text {drift }}$ is roof drift as the dependent variable that varies from 0 to $10 \%$ as recommended is AISC Seismic Provisions, $\mathrm{h}$ is story height, and $\mathrm{K}$ is the lateral stiffness the braces apply to the system. [AISC 2005] 


\subsubsection{System Weight}

Weight of the system is proportionate to the frequency of the system, geometric properties, and brace properties. As stated in the previous analysis of the single frame can be representative of the response of an entire structure. Since weight is proportionate to frequency, a range of system weights can represent a range of system frequencies, and therefore represent a range supported stories above the CBF. For simplicity all of the system mass is lumped into equal portions to the upper corners of the CBF. For the braces under consideration and over a natural period range of 0.1 to 2.0 seconds at 0.1 second increments, a system weight matrix can be calculated with the following equation.

$$
m=\left(\frac{T}{2 \pi}\right)^{2} *\left(\frac{2 g E \cos \theta^{2} B^{2}\left[1-\left(1-\frac{2 t}{B}\right)^{2}\right]}{L_{B}}\right)
$$

Where $T$ is the natural period, $g$ is gravity, $E$ is Young's Modulus, $\theta$ is from the geometry of the braces within the bay, $B$ is the width of the square brace, $t$ is the wall thickness of the brace, and $L_{B}$ is the brace length. Refer to Figure 2.1 for a visual representation, if the figure the lumped masses are represented with circles at the beam-column interfaces of the CBF. 


\subsection{Running the Model on OpenSees}

Everything herein for sequential (e.g. OpenSees.exe) is ran on a Windows 7

Professional Service Pack 1 OS. Processes and program availability may vary for other Windows versions, Macintosh OS, Linux OS, and Unix OS. Everything ran in parallel (e.g. OpenSeesMP.exe) is ran through OpenSees Laboratory on The George E. Brown, Jr. Network for Earthquake Engineering Simulation (NEEShub) a program of the National Science Foundation (NSF). Processes required to conduct analysis and the preloaded software downloaded to severs and clusters may vary drastically. Users must ensure that the servers/cluster being used has the necessary software installed to run respective simulations.

The rest of this section discusses which OpenSees executable is most appropriate. It also weights computational efficiency versus programing difficulty. And provides an introduction to parallel computing and how parallel computing was used in this thesis.

\subsubsection{Choosing between OpenSees, OpenSeesSP, and OpenSeesMP} OpenSees.exe is the default executable used to run the OpenSees framework. The object-based framework uses two interpreters, Tcl/Tk and OpenSees. ActiveTcl v8.5 creates most of the base string commands and invoking methods need for model creation and analysis. The OpenSees interpreter adds specific commands to run structural and geotechnical analysis, such as materials and solvers. OpenSees.exe is run with a sequential application and is therefore ideal for smaller models that are run on personal computers [McKenna and Fenves 2007; McKenna 2014]. The majority of the data collected in this thesis was through OpenSees.exe due to its simplicity and ease of running the data locally. 
OpenSeesSP.exe is similar to OpenSees.exe as it will parse and execute the script in a sequential application. However, it uses a parallel application for element state determination and for solving equations. To partition the work, a master processor interprets the input script and then sends commands to slave processors. Few changes need to be made to the input script so OpenSeesSP is only slightly more difficult to code and run. Solvers: Mumps, Petsc, and SuperLU can be used to improve efficiency with quality in descending order but with difficulty to implement also in descending order. OpenSeesSP use is ideal for a large model being run on a single computer with a multicore processor or on a cluster of machines. [McKenna and Fenves 2007; McKenna 2014]

OpenSeesMP.exe use is ideal for parameter studies on moderately sized models. Its goal is to discretize the work up over the number of available processor and then run like the OpenSees.exe. OpenSeesMP has several more additional commands, including those stated in OpenSeesSP, particularly regarding how the processor communicate and work with one another. It is more difficult to code and requires substantial effort and skill to optimize, but if properly utilized parallel processing can result in drastic speed up times [McKenna and Fenves 2007]. OpenSeesMP was used for about a third of the data collected in this thesis and was ran over venues in the Teragrid. Further discussion of parallel computing and the supercomputer venues can be found in Section 2.3.3. 


\subsubsection{Computational Cost}

Running Incremental Dynamic Analysis, discussed in detail in Section 3.3, is very computationally expensive. This thesis covers several parameter studies for each of the 4 brace types, over a suite of 20 earthquakes, with 20 system frequencies per earthquake, an average of about 125 incremental steps to get to the desired ductility per frequency, and 8000 time steps per incremental step (40 seconds of the earthquake with a dt of 0.005 seconds). That is about $1.6 \mathrm{E}+09$ data points for full IDA of a single parameter. Figure 2.8 below shows an example of how much time it can take to analyze approximately on quarter of a control study (e.g. no parameters were evaluated).

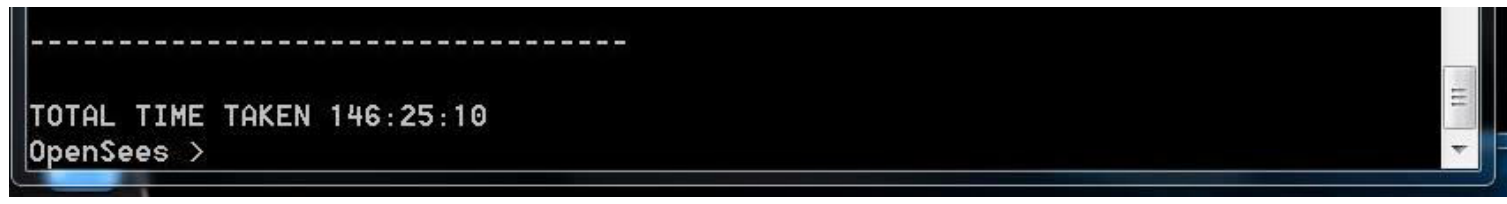

Figure 2.8: OpenSees run locally on an MSI GT60 2OKWS.

This run was conducted locally with OpenSees.exe on an overclocked MSI GT60 2OKWS. Full stats of the MSI are given in Table 2-2 below for a means of computational comparison. This computer gets roughly 32 GFlops/sec.

Table 2-2: Meaningful stats when considering computation time.

\begin{tabular}{|l|l|}
\hline Computer Model & MSI GT60 2OKWS 674US \\
\hline Processor Chip & $\begin{array}{l}\text { Intel Core i7-4800MQ, 2.7 GHz (Max 3.7GHz), 6MB } \\
\text { Smart Cache }\end{array}$ \\
\hline Memory Drive & Crucial M550 SATA3 6Gb/s 256GB mSATA SSD \\
\hline Video Card & 4GB NVIDIA Quadro K3100M GDDR5 \\
\hline
\end{tabular}


Where the processor chip (CPU) has a substantial impact on the speed of running the calculations over its cores. A solid state drive (SSD) is much faster at reading and writing to files than a HDD. And if properly coded, OpenSees can utilize memory to run analysis on the computer's video card (GPU).

Running the same code on a slower computer will take longer. No study was conducted in this thesis to benchmark OpenSees on different computers, but several computational benchmark studies have been conducted online. Refer to any of those studies for an approximate correlation of computational times for different rigs. Besides the computing power of the system, the type of computing effects computational time. For example, if the fatigue model is being considered then computational time will greatly increase. The fatigue model will weaken the system, resulting in less incremental steps, but the computational time per step increases due to the added state determination calculations for the fatigue material.

The ease of running OpenSees locally is invaluable. Approximately six executables can be run simultaneously without effecting each other or causing system instability. However, running multiple executables will use the majority of that computer's CPU, so minimal additional processes can be run. Meaning to run OpenSees locally, the computer should be dedicated to solely running OpenSees. Another annoyance is that OpenSees will often crash without reason. So if code is running for an extended amount of time, it needs to be checked in frequent intervals to ensure that the program hasn't crashed. Computation cost should be a major consideration when choosing which executable is most appropriate for running analysis. The next section covers running OpenSees with parallel computing. 


\subsubsection{Parallel Computing}

Parallel computing is the process of distributing the computational efforts required to run a script over many processors. Clusters of computers in parallel can have tens to hundreds of processors and parallel supercomputers can have several thousand processors. With an efficiently coded script, the time to run the script can be greatly reduced. Parallel computing improvements over the years are allowing for more sophisticated simulations of the structural performance under realistic earthquake excitations. [McKenna and Fenves 2007; McKenna 2014]

\section{Performance Development}

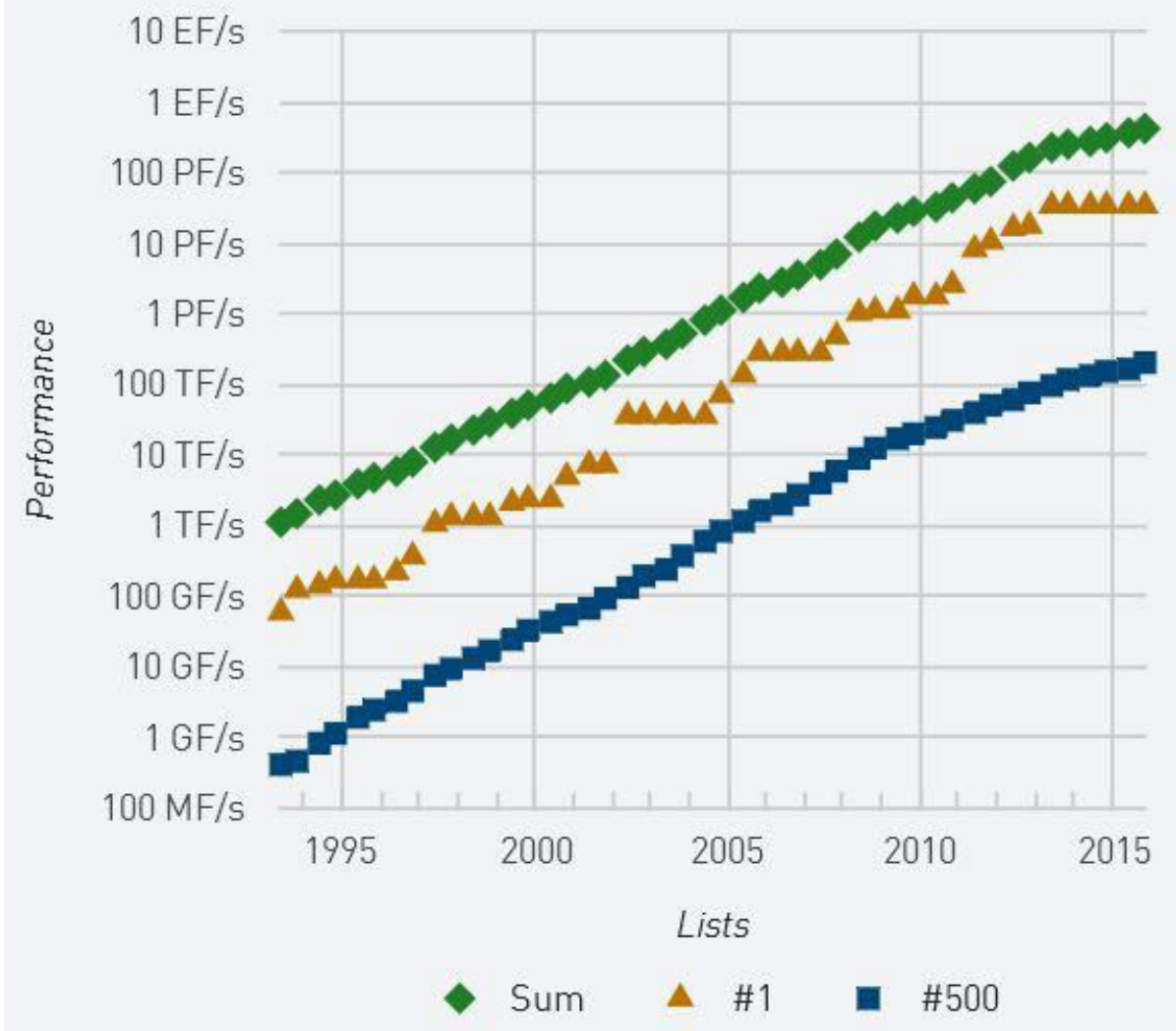

Figure 2.9: Flops/sec of the 500 best supercomputers in the world [Image from www.top500.org]. 
Figure 2.9 above shows the improvements in supercomputing throughout the world over time. The data is comprised of the top 500 fastest computers in the world at a given time, where speed is determined by their Flops/s (floating point operation per second). The figure shows that the world is improving linearly on a logarithmic scale. With so much available computer power it does not make sense to not utilize parallel computing for research.

A portion of the data collected in this thesis was through parallel processing using NEEShub and the Teragrid. Any person with an .edu email account can create an account on NEEShub, restrictions to non-US citizens may apply. NEEShub supports many applications for research efforts. Refer to [NEEShub 2009] for more on these applications. The main NEEShub applications used in this thesis is OpenSees Laboratory, Batchsubmit, PEN 2.4, SynchroNEES, and Workspace. Where OpenSees Laboratory and Batchsubmit are used for data submission to the supercomputers/servers. PEN 2.4 and SynchroNEES are used for data reception. And Workspace is similar to a Command Prompt. [McKenna et al. 2010; Dong et al. 2010; McLennan et al. 2010; Kisseberth 2015; Rodgers et al. 2011]

Data submission consists of uploading all required scripts, choosing a venue, and selecting input values for the venue. OpenSees Laboratory and Batchsubmit can be used interchangeably as the only difference is the interface. Where OpenSees Laboratory is a GUI and Batchsubmit is script based. Instructions on how to set up and run each application are in Appendix D. NEEShub has local servers to run analysis but they are rather small and slow, relative to the supercomputers on Teragrid. It is recommended to request access to High Performance Computing (HPC) with a support ticket to the site. 
HPC is grant access to the Teragrid. The Teragrid, now Extreme Science and Engineering Discovery Environment (XSEDE), is a network of supercomputer access sponsored by the NSF [Towns et al. 2014]. Once access to the Teragrid is approved, analysis can be ran on the available venues listed in Table 2-3 below. Where the columns are total number of CPUs (ncpus), number of nodes (nn), and processors per node (ppn).

Table 2-3: Available venues on the Teragrid.

\begin{tabular}{|l|l|l|l|l|}
\hline Venue & ncpus & nn & ppn & Walltime \\
\hline Stampede & $4096(\max )$ & $256(\max )$ & $16(\max )$ & $24: 00: 00$ (max) \\
\hline Kraken & $\begin{array}{l}512(\min ) \\
12(\max )\end{array}$ & $42(\max )$ & $12(\max )$ & $24: 00: 00$ (max) \\
\hline Hansen & $48(\max )$ & $12(\max )$ & $4(\max )$ & $720: 00: 00$ (max) \\
\hline osg & $\sim 60000$ & NA & NA & $24: 00: 00$ (default) \\
\hline Carter & $64(\max )$ & $4(\max )$ & $16(\max )$ & $72: 00: 00$ (max) \\
\hline Local (NEES) & $16(\max )$ & $1(\max )$ & $16(\max )$ & $24: 00: 00$ (max) \\
\hline
\end{tabular}

Note that access to Stampede and Kraken require additional approval for walltime. In this thesis supercomputers Carter and Hansen of Purdue University were primarily used. These venues seemed more reliable than the others but still had several errors, refer to Appendix $D$ for further discussion. Near the beginning of summer in 2015 all of the venues became much less responsive, hopefully this was due to the transition from Teragrid to XSEDE. 
Data reception consists of downloading output files from analysis. SynchroNEES and PEN 2.4 can be used interchangeably and are both GUIs. SynchroNEES is an executable that is downloaded to a computer and remotely access NEEShub. SynchroNEES is easy to use but is very susceptible to crashing. It is best suited for downloading a small quantity of files. PEN 2.4 is an application on NEEShub that is used to mass download files into a single zip file. Detailed write-ups on each can be found in Appendix D. If a large quantity of analysis is being ran than it is recommended that users submit a support ticket requesting allotted NEEShub memory be increased from the 1GB default to 10GB.

The efficiency gained from running a program in parallel is largely dependent on how well the script is coded. The script could experience race conditions, where one processor is reading data before another processor is finished writing. This can be fixed with a barrier command but barriers can lead to deadlock, where code can get stuck forever at that barrier. Poor performance of a single processor could hold up other processors. There could be load imbalance, where some processors idle while others are doing all of the work; typically caused by processes having different amounts of work (e.g. one processor running gravity loads while the other runs transient analysis). There could be communication issues between processors, particularly in clusters [McKenna 2014]. Script that is run in parallel is much more difficult to code and requires several iterations to optimize, assuming the coder has the patience and skill. 


\subsection{External Programs}

Conducting analysis of the model requires the use of external programs in addition to OpenSees. The plethora of external programs that may be used/needed aid in preprocessor, processor, and postprocessor steps. The preprocessor step includes all calculations needed as input values to run OpenSees. This includes defining and evaluating loading patterns, ground motion suites, characterizing the ground motion suite, and intermediate steps of analysis. The processor step is running OpenSees itself. It is included in this section because external programs are used to analyze the source code of OpenSees. The postprocessor step, referred to here as data interpretation, includes the programs used to analyze the outputs of the processor step. This step also includes programs used to graphically represent the data.

Programs listed in the preprocessor, processor, and postprocessor sections can be thought of as required programs, as they are needed or must be substituted in order to continue analysis. Other programs can be used to aid in organization of data, such as Windows PowerShell and .tar file compression. PowerShell is used for the renaming of a mass quantity of files. tar is used to compress mass quantities of data to save hard drive space; programs like WinZip can extract compressed files.

Another set of programs used check the results given from OpenSees. This step is particularly important when discussing the validity of results. This set of programs include SAP 2000, RISA, and many other structural analysis programs. Note that many of these programs do not have materials that model the effects of low-cycle fatigue. Note that to run OpenSeesSP and OpenSeesMP the system/cluster will need MPICH2. Windows OS may need SDK in order to run MPICH2. [McKenna and Fenves 2007; McKenna 2014] 


\subsubsection{Preprocessor Inputs}

The OpenSees analysis ran in this thesis requires many preprocessor inputs. These inputs include load patterns, ground motion files, values characterizing inputted ground motion files, values from previously ran analysis, and the tcl scripts defining the model. These inputs require several different programs.

Microsoft Excel is heavily used in preprocessor inputs due to its availability and ability to run calculations for thousands of points. Excel is also familiar and easy to use for most users. It has the ability to call other sheets which helps keep work visually organized, crucial for avoiding errors. The ability to merge numeric values with alpha characters is key for callouts that require alpha-numeric strings. Excel is used to fill most tcl scripts that are in matrix form.

Refer to Section 3.3.1 for the earthquake suite used in transient analysis. OpenSees scripts in this thesis are set up to read at2 file extension for original ground motion files. The original ground motion files need to be preprocessed in order to get spectral displacement, a component of the calculation of the initial scaling factor. This value is calculated in Excel but is checked with SeismoSignal, an earthquake evaluation software. Refer to Section 3.3.2 for how to calculate the initial scaling factor.

OpenSees scripts are all .tcl extension files. These scripts contain all of the code to define the model, mass, element properties, loading, analysis type, and solvers. It is recommended that these scripts be written with high quality text editors like Notepad ++ or UltraEdit. These editors have the ability to read most text file extensions and can be used to edit/compile VC++ code. 


\subsubsection{OpenSees Source Code}

Source code can be crucial in determining the appropriate solvers or materials for analysis. By having access to the source code, users can know exactly how the software comes to a solution and what assumptions it makes to get to that solution. Most programs have manuals regarding this information but they often summarize and potentially leave out key information. Another benefit of open source software is the ability for users to modify the code to fit their needs.

There are two primary ways to view OpenSees' source code. The first is to view it anonymously on OpenSees' website under Source in the Developer tab. This location can also be reached by entering:

svn co svn://peera.berkeley.edu/usr/local/svn/OpenSees/trunk OpenSees

in Window Command Prompt. To get to the useful portion of the code go to trunk/SRC/ and view the item of interest. Each item has an .h and a .cpp file extension. Header files (.h extension) act as directory files that communicate with other files. Code files (.cpp) contain the variables and functions that define the unique item, and are therefore the file of interest. [OpenSees Subversion Repositories]

The second method to view the source code is to download it as a VC++ project. A program that can download SVN is required, the OpenSees website recommends TortoiseSVN. Once the SVN repository is downloaded a program like VisualStudio VC++ can be used to view and modify branches of the project. GitHub is a free online alternative to VisualStudio VC++. OpenSees source code is used in this thesis to look at Geometric and Material Nonlinearities during state determination within Steel02 and the Fatigue Material. Refer to Sections 3.4 and 3.5 for further discussion. 


\subsubsection{Data Interpretation}

There are many possible output values given by OpenSees depending on which recorders are used in the script. Refer to the OpenSees Manual for all of the recorder types [McKenna and Fenves 2004]. The main recorder used in this thesis is horizontal roof displacements used to calculate ductility in the system. Force and stress recorders were also used to check state determination. Refer to Section 3.3 for the calculation of ductility. In this thesis ductility, and the R-Factor, was calculated in Matlab. The Matlab script could be eliminated by adding that script to the OpenSees protocol. This may make the code more efficient but may introduce race conditions.

Once the R-factors are calculated from ductility, comparison is conducted with visual representation. Excel and Matlab are the two main programs used for plotting data in this thesis. These are two commonly used programs for plotting data, but there are many available programs that have similar capabilities. $\mathrm{R}$ is another commonly used visual representation program used in academic research.

After data has been analyzed it can be compressed to save space on hard drives. The compression method used in this thesis is tar. No external program is needed for tar compression as it is done through Windows Command Prompt. A write up on how to use tar compression is found in Appendix B. If the data needs to be accessed again, the files can be extracted using WinZip, or equivalent software. 


\section{Analysis Methods}

This chapter discusses the analysis methods applied to the CBF model. Methods include pushover, cyclic, Eigen, and incremental dynamic analysis (IDA). Each section contains the reasons why that analysis type is chosen and how it effects the results. Often multiple methods are performed for each parameter study to help given a means of comparison, typically pushover and IDA are run in each study. This way the simple pushover method can help validate the more complex IDA.

Eigen analysis is simply more of a check and is too short to warrant its own section. Eigen checks are done during every types of analysis to verify the frequency and mass of the system. Figure 3.1 below shows little change in weight during pushover analysis in yielded sections.

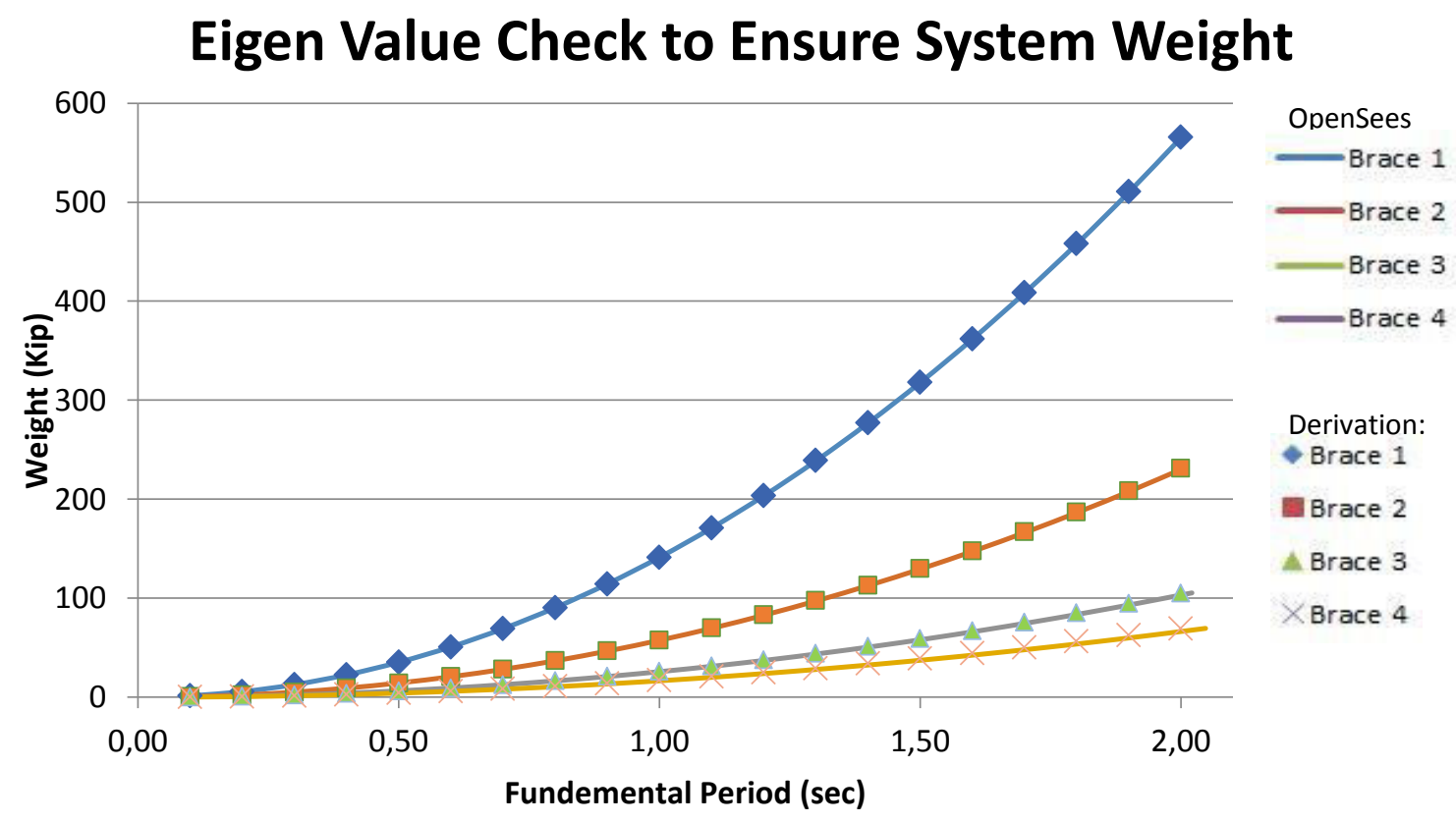

Figure 3.1: Eigen value check during pushover analysis.

This chapter also investigates geometric and material nonlinearities, as they have an effect on how fast and accurately non-elastic analysis converges. 


\subsection{Pushover Analysis}

Pushover analysis is one of the simplest methods to observe failure in computation simulations and in real life experiments. Useful data that comes from pushover analysis includes the ultimate lateral capacity which is shown as peak base shear, $\mathrm{V}$, and result in the roof displacement associated with the first failure, $\cup_{y}$, in which occurs when the data becomes nonlinear. Pushover can also give useful insight in post yielding behavior, such as strain hardening.

The physical interpretation of pushover can be seen in Figure 3.2 below, where a force is applied at roof level and continues so there is a constant displacement gradient. In the figure $T=R_{y} F_{y} A_{g}$ represents the lateral resistance contribution the tension brace adds: where $R_{y}$ accounts for expected yield stress values, $F_{y}$ equals the yield force, and $A_{g}$ is the gross cross-sectional area of the brace. $C=F_{c r} A_{g}$ represents the lateral resistance contribution the compression brace adds, where $F_{c r}$ is the critical load. Note the compression lateral resistance represents the maximum resistance the compression brace adds, residual post-buckling strength is on about $30 \%$ of the nominal strength [Qu 2014; AISC 2005].

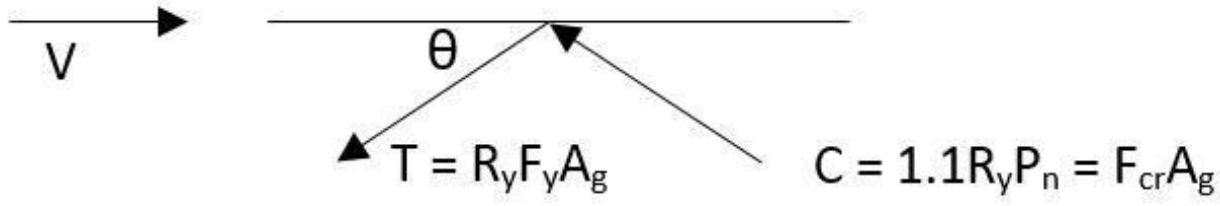

Figure 3.2: Lateral resistance contributions. 


$$
\begin{gathered}
V=F_{y} B^{2}\left[1-(1-2 t / B)^{2}\right] \cos \theta+F_{c r} B^{2}\left[1-(1-2 t / B)^{2}\right] \cos \theta \\
V=F_{y}\left[1+\frac{F_{c r}}{F_{y}}\right] B^{2}\left[1-(1-2 t / B)^{2}\right] \cos \theta \\
1+\frac{F_{c r}}{F_{y}}=\left\{\begin{array}{c}
1+0.658^{\lambda^{2}}, \text { for } \lambda \leq 1.5 \\
0.877 / \lambda^{2}, \text { for } \lambda>1.5
\end{array}\right. \\
\text { Where } \lambda=\frac{k L}{\pi r} \sqrt{\frac{F_{y}}{E}}
\end{gathered}
$$

Equation 5 above combines the lateral resistance contributions of each brace and with some manipulating is represented only in terms of properties of the brace. This equation can give insight on how slenderness effects each of the resistance contributions. Appendix E contains supporting calculation for Table 3-1 below, which shows the percent capacity imbalance between the tension and compression members.

Table 3-1: Force imbalance for varying slenderness ratios.

\begin{tabular}{|c|c|c|c|c|}
\hline & Brace 1 & Brace 2 & Brace 3 & Brace 4 \\
\hline $\mathrm{kL} / \mathrm{r}$ & 50 & 100 & 200 & 300 \\
\hline Compression & $45.8 \%$ & $33.8 \%$ & $12.0 \%$ & $5.7 \%$ \\
\hline Tension & $54.2 \%$ & $66.2 \%$ & $88.0 \%$ & $94.3 \%$ \\
\hline
\end{tabular}

As seen in Table 3-1 above, lower slenderness values support a more even distribution of force over both braces. This is supported with the AISC Seismic Provisions which state, "The imbalance between tension and compression capacity for stocky braces is less than for slender braces." [AISC 2005] In stockier braces it is predicted that first failure mode will occur from yielding where in slender braces it is predicted that first failure mode will occur from buckling. Braces in compression will buckle at lower levels of frame drift than when braces will yield in tension. Therefore, the compression braces will have likely buckled by the time the braces yield in tension. [Qu 2014; AISC 2005] 
The base shear and corresponding displacement at first yield is used in several calculations throughout this thesis. With multiple failure criteria for the different braces, it is crucial that an appropriate value of where yield occurs is selected. Figure 3.3 below shows each of the braces undergoing pushover analysis.

\section{Effect of Brace Size on Base Shear}

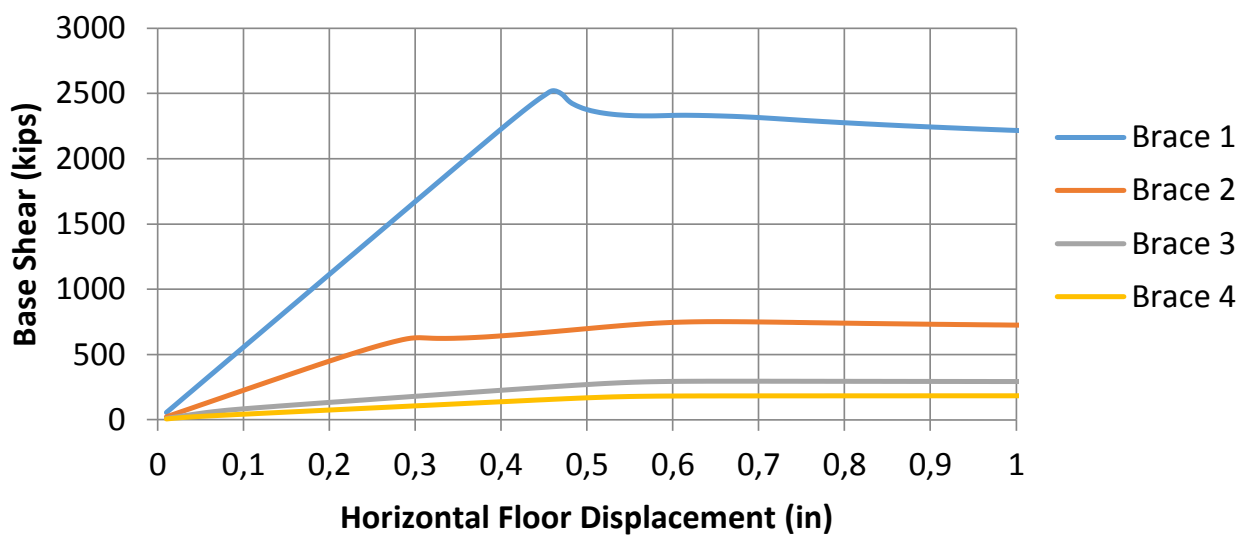

Figure 3.3: Base shear using pushover analysis.

In Figure 3.3 above it is unclear exactly where first yield occurs. Therefore, each brace is analyzed to determine the maximum base shear, the displacement corresponding maximum base shear, at twenty percent change in slope in the base shear function, at twenty percent change in slope in the axial tension function, and at twenty percent change in slope in the axial compression function. Results are summarized in Table 3-2 and are visualized in Figures 3.4.a to 3.4.d. First yield was decided to occur at twenty percent change in slope in the axial tension function as it gave consistent values that seemed to best represent the system. Note in Figure 3.4.d, Brace 4 completely collapses after 3.5 inches of roof displacement. 
Table 3-2: Summary of displacement associated with first yield under multiple criteria.

\begin{tabular}{|c|c|c|c|c|c|c|c|c|}
\hline & \multicolumn{2}{|c|}{ Brace 1} & \multicolumn{2}{|c|}{ Brace 2} & \multicolumn{2}{|c|}{ Brace 3} & \multicolumn{2}{|c|}{ Brace 4} \\
\hline & $B=12.5$ & .86 & $B=6.5 T$ & 71 & $B=3.5$ & & $B=2.5$ & 68 \\
\hline $\mathrm{Kl} / \mathrm{r}$ & 50 & & 100 & & 200 & & 300 & \\
\hline Max Vx & 2520.9 & kip & 751.9 & kip & 295.6 & kip & 190.3 & kip \\
\hline Max Vx & 0.46 & in & 0.65 & in & 0.67 & in & 4.01 & in \\
\hline Slope change & 0.45 & in & 0.28 & in & 0.57 & in & 0.55 & in \\
\hline Tension & 0.45 & in & 0.63 & in & 0.57 & in & 0.55 & in \\
\hline Compression & 0.46 & in & 0.29 & in & 0.37 & in & 0.73 & in \\
\hline
\end{tabular}
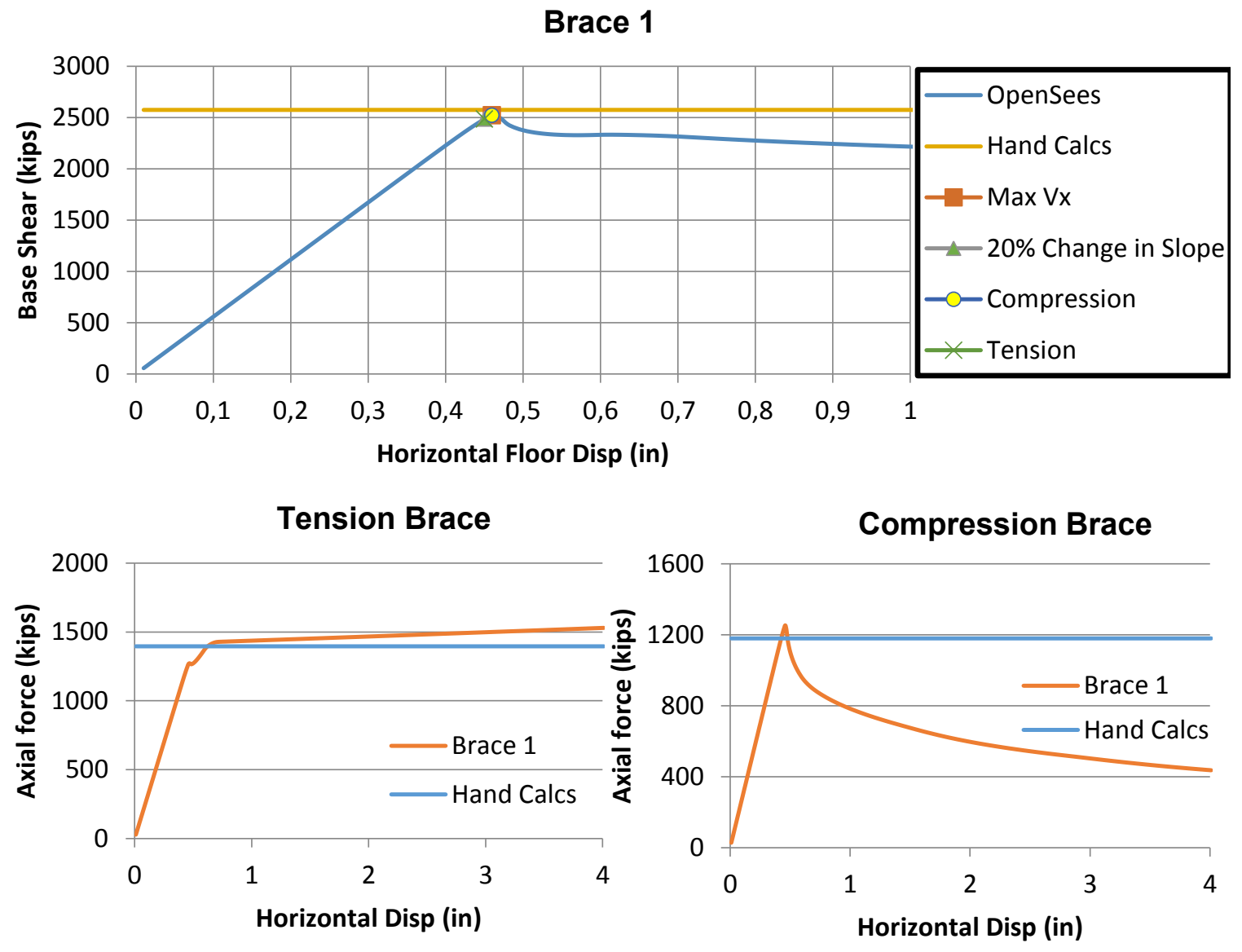

Figure 3.4.a: Base shear and axial load in Brace 1 under pushover analysis. 
Brace 2

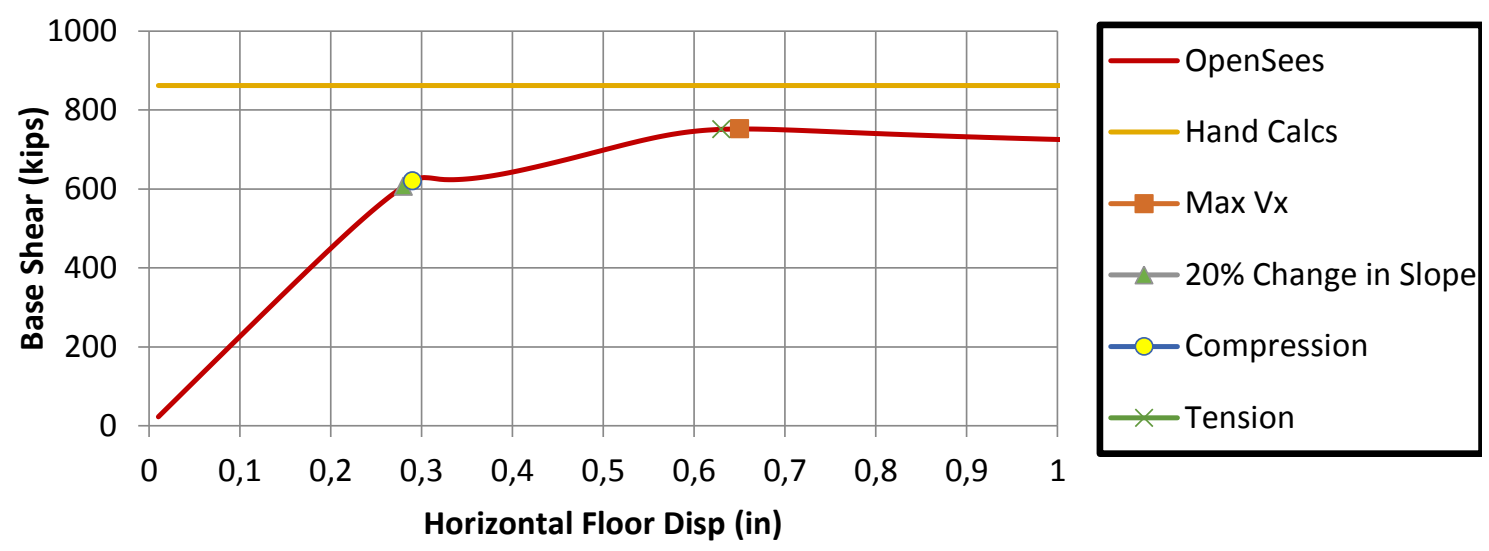

Tension Brace

Compression Brace
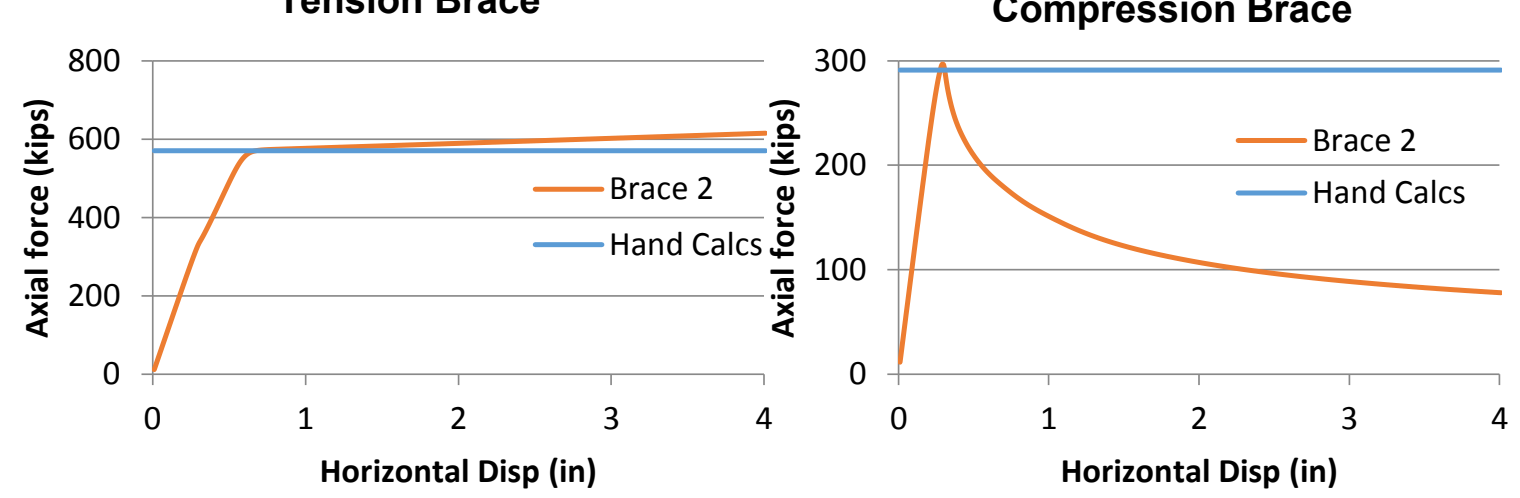

Figure 3.4.b: Base shear and axial load in Brace 2 under pushover analysis. 

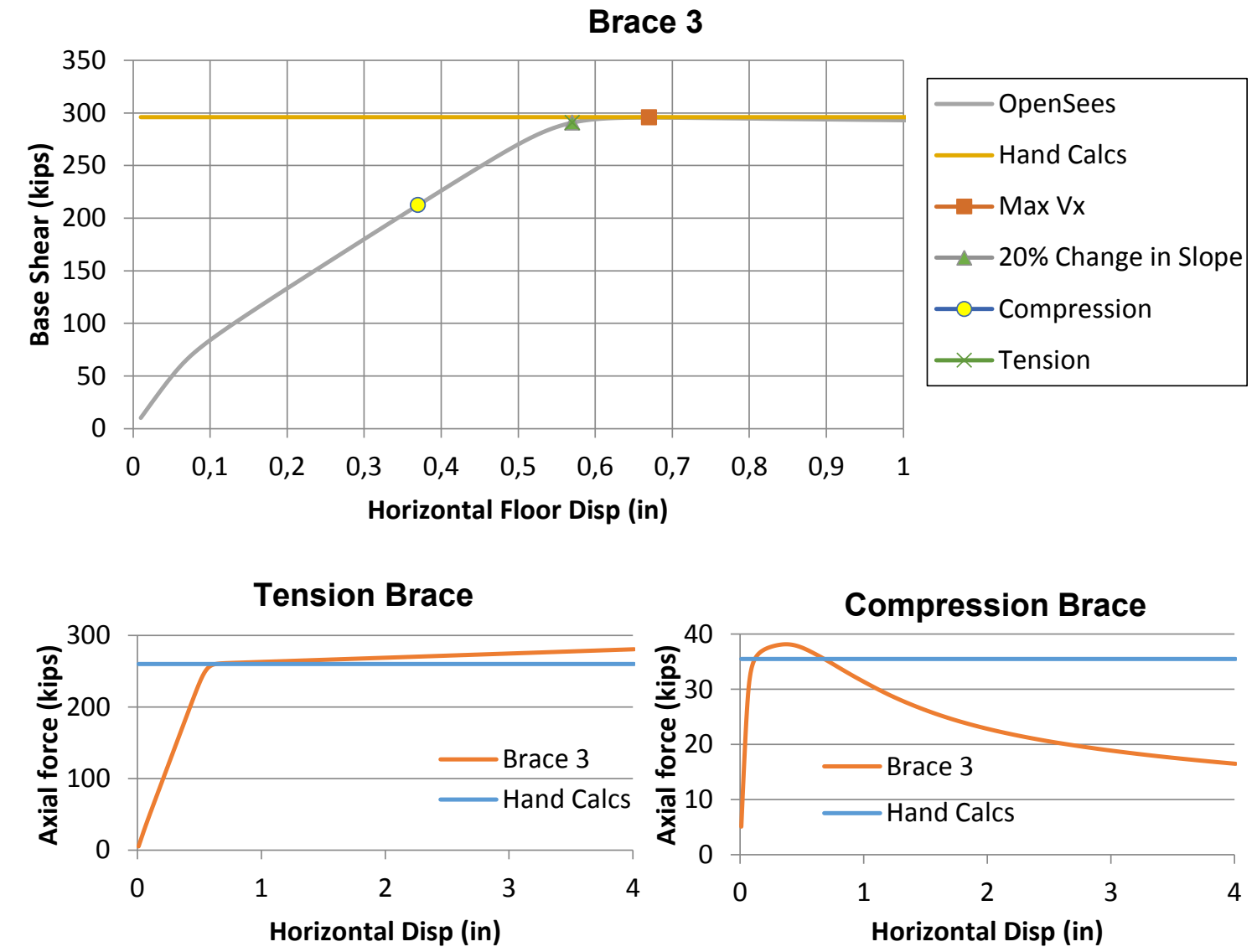

Figure 3.4.c: Base shear and axial load in Brace 3 under pushover analysis. 

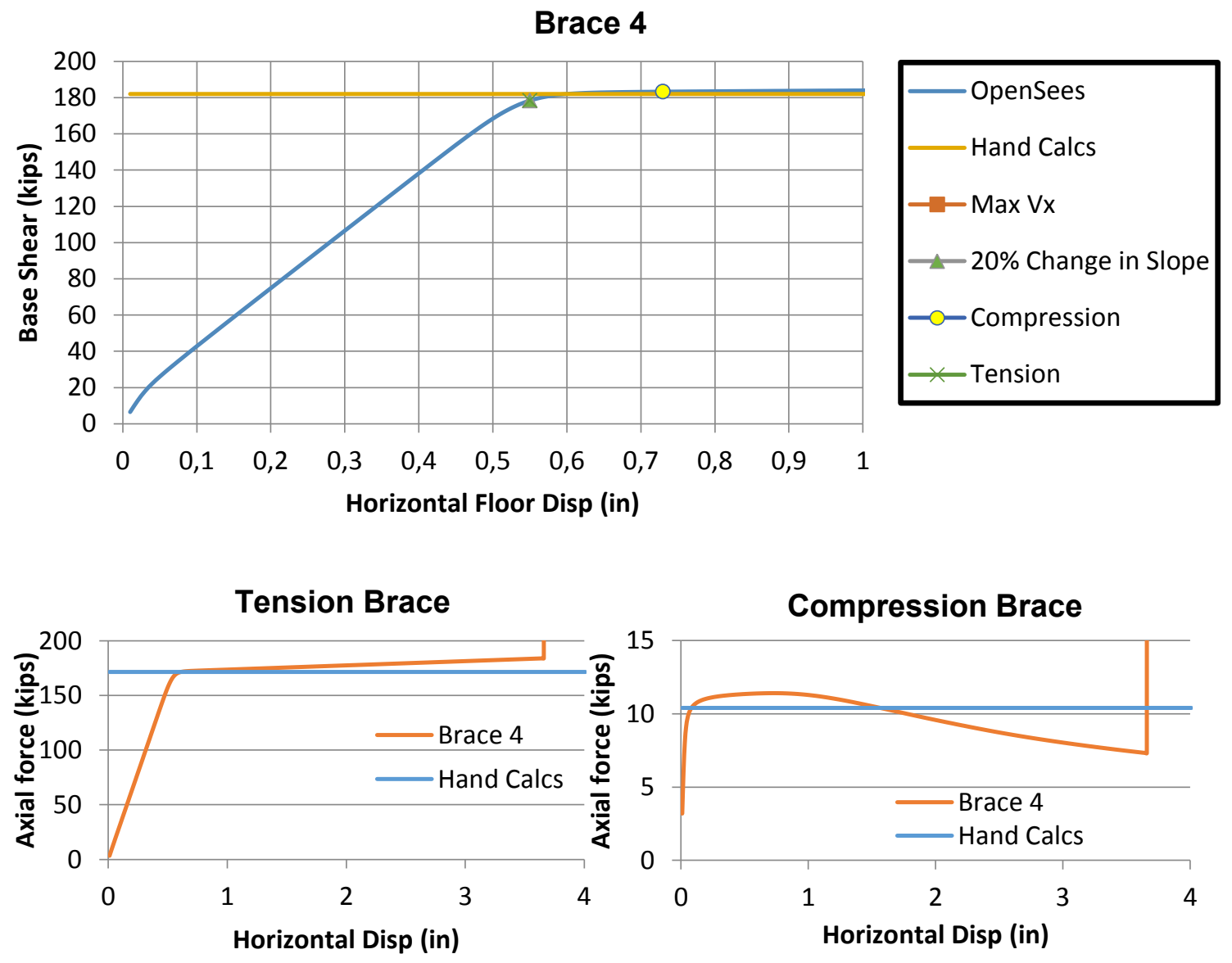

Figure 3.4.d: Base shear and axial load in Brace 4 under pushover analysis. 


\subsection{Cyclic Analysis}

Cyclic analysis is another method commonly used in computer simulation and

occasionally in real life experiments. This method gives the ultimate lateral capacity with the associated displacements but also gives insight on post failure behavior and energy dissipation. Little cyclic analysis was performed in this thesis due to the large amount of hysteretic testing in other papers [Huang and Mahin 2010; Li and Fahnestock 2013; Uriz and Mahin 2008; Krawinkler 2009; Sanchez-Zamora 2013]. Though Figures 3.6.a to 3.6.d are included on the following pages to give insight on the effect slenderness has on the hysteretic curves.

In the figures, Brace 1 and 2 have relatively large areas under the curve. This insists that they have better energy dissipation than the more slender braces. This is confirmed in the AISC Seismic Provisions which state that stockier braces provide better energy dissipation capacity than slender braces [AISC 2005]. For further information on the hysteretic response of the fatigue material used in the braces refer to Uriz and Mahin 2008. The cyclic loading protocol is shown in Figure 3.5 below, several studies have been conducted on the loading protocol [Krawinkler 2009].

\section{Cyclic Loading Protocol}

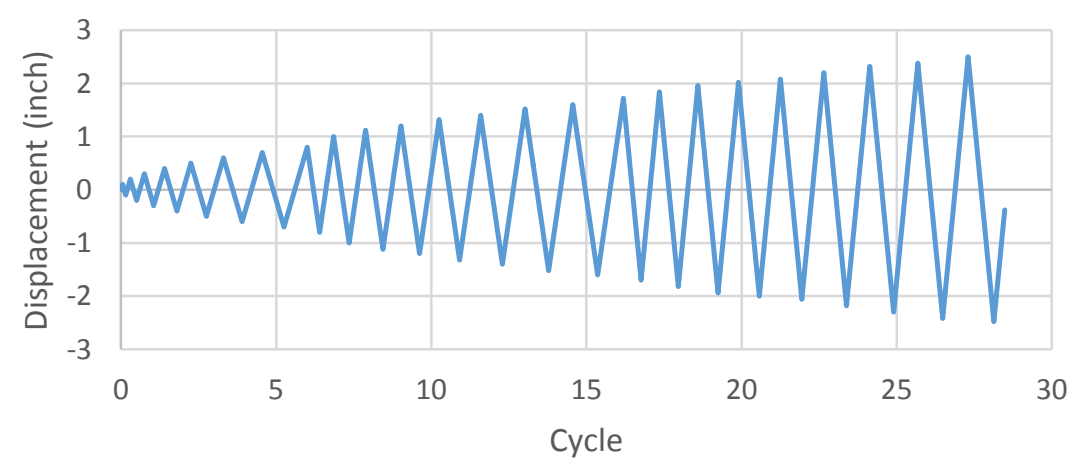

Figure 3.5: Cyclic loading protocol for steel frames. 


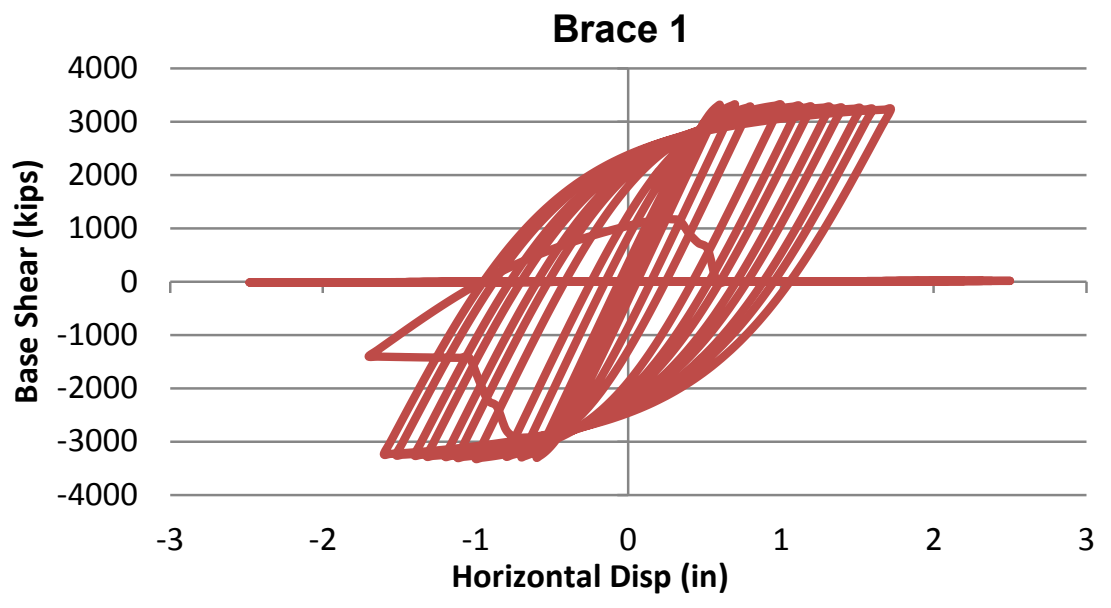

Axial Force on left brace

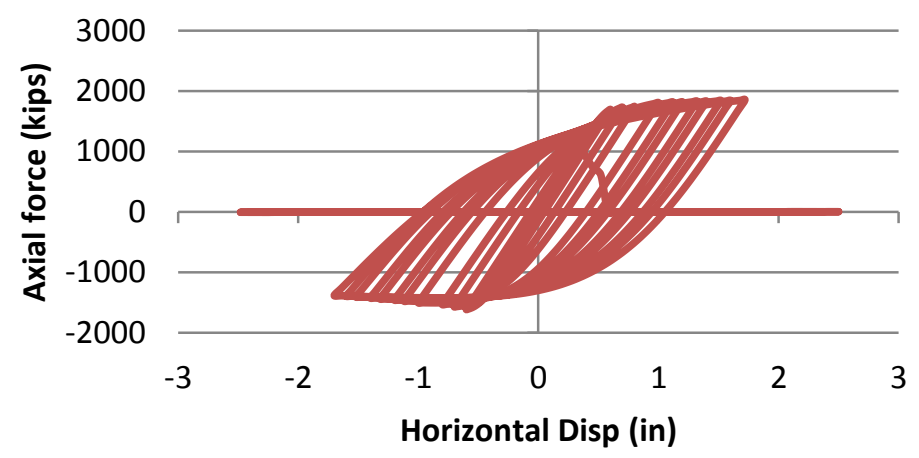

Axial Force on right brace

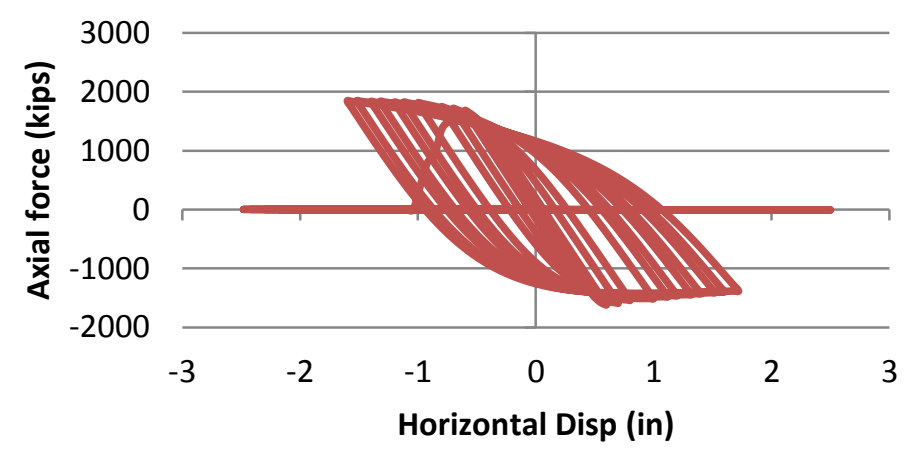

Figure 3.6.a: Hysteretic base shear and axial load in Brace 1 under cyclic analysis. 

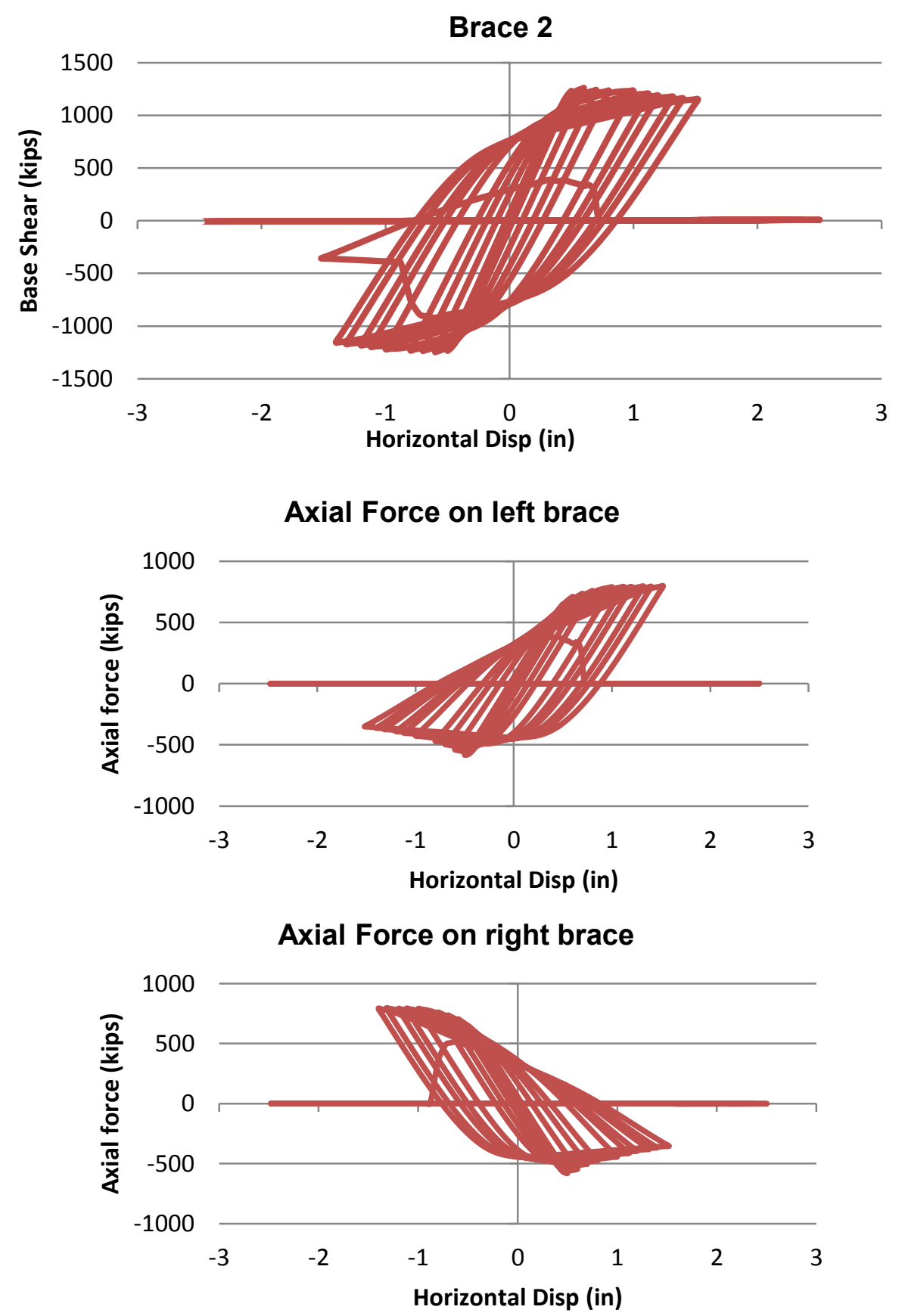

Figure 3.6.b: Hysteretic base shear and axial load in Brace 2 under cyclic analysis. 


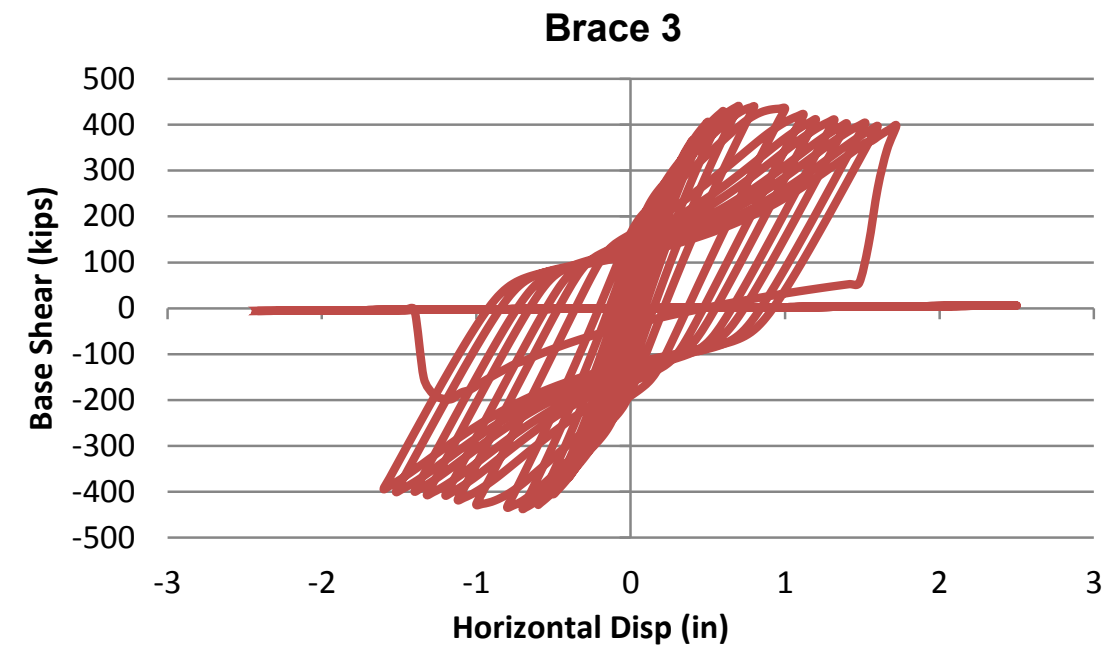

Axial Force on left brace

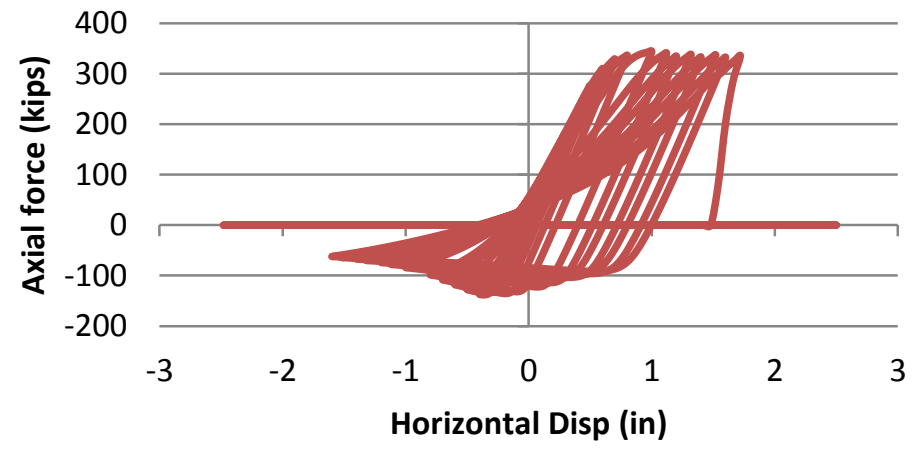

Axial Force on right brace

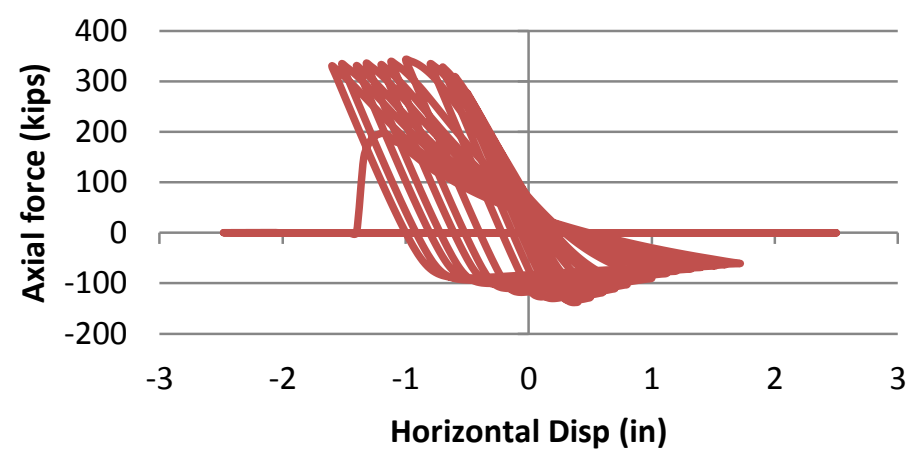

Figure 3.6.c: Hysteretic base shear and axial load in Brace 3 under cyclic analysis. 

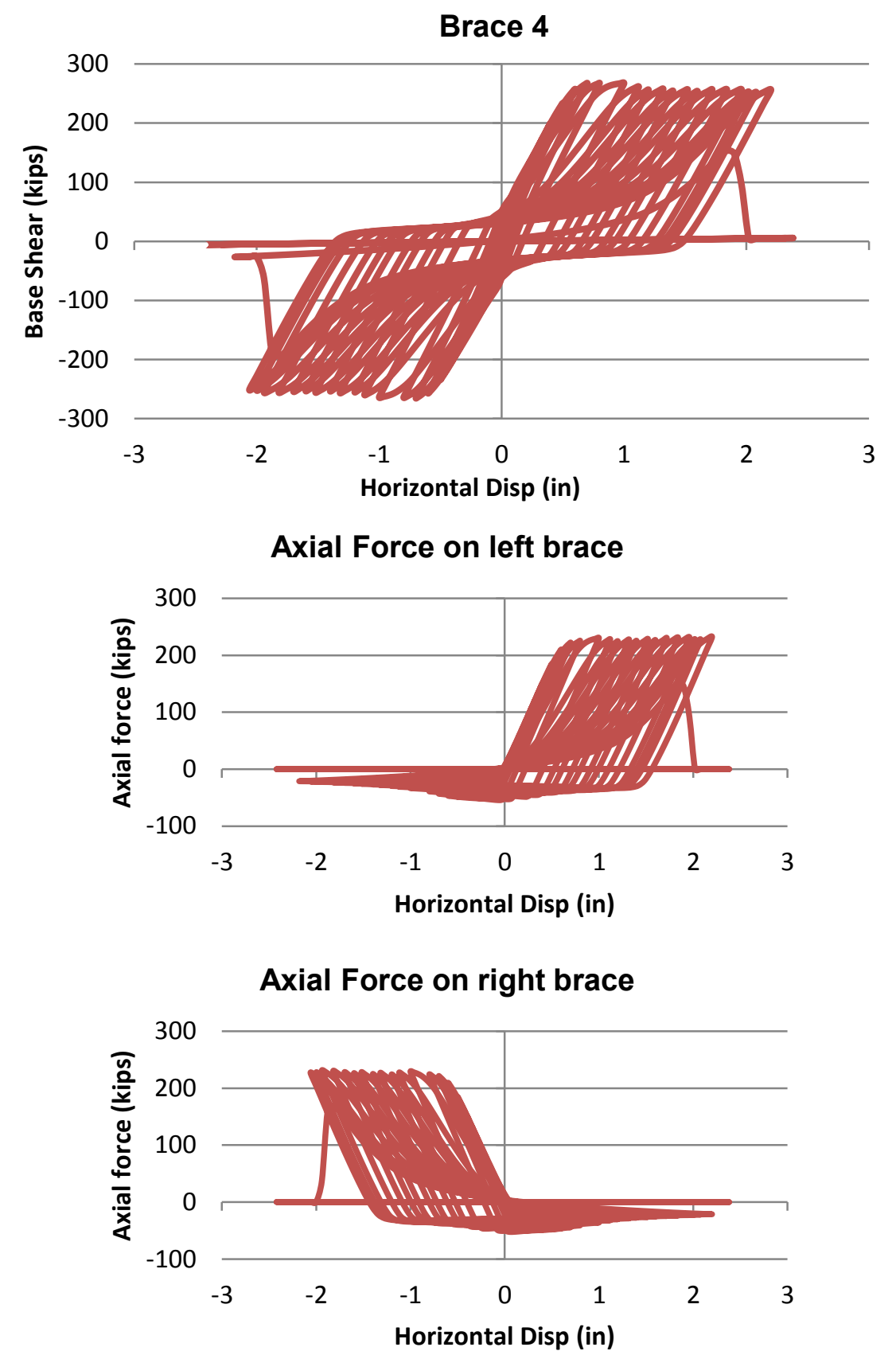

Figure 3.6.d: Hysteretic base shear and axial load in Brace 4 under cyclic analysis. 


\subsection{Incremental Dynamic Analysis}

Incremental Dynamic Analysis (IDA) is a common method used to determine the response of a structure under dynamic loading, whether seismic or other time-dependent loadings. Real life dynamic experiments are very difficult to replicate due to the randomness of earthquakes, therefore full scale dynamic analysis is typically only conducted using computational simulations. These simulations are very computationally expensive and therefore are typically solely used in research.

There are two typical methodologies used in IDA. The first keeps the inputted earthquake the same and incrementally weakens the structure to get the full response for an earthquake. This is the typical methodology used since it uses real unaltered earthquakes while analyzing the response of a range of structures with varying stiffnesses. The second keeps the system exactly the same to ensure that the frequency of the structure isn't altered, this gives the full analysis for a given system. The earthquake record is then incrementally strengthened to get a range of responses for the single system. Since the purpose of this thesis is to capture the effects that fatigue has on braces with differing slenderness ratios, the second methodology of IDA is used throughout analysis. [Vamvatsikos and Cornell 2002]

For this thesis, comparison of the different parameters are measured through their effect on the Response Modification Factor (R-Factor), which is calculated with ductility. The goal ductility throughout is for the system to reach a ductility of 6 . A ductility of 6 is chosen to represent a highly ductile system. An R-Factor is calculated over a period range of 0.1 seconds to 2.0 seconds (frequency range of 10 to $.5 \mathrm{Hertz}$ ) in 0.1 second increments. This allows for comparison of a single brace representing twenty different systems (since the frequencies of the structure vary due to a change in mass). 
To conduct IDA on the model a ground motion, brace, frequency range, the range of incremental steps and any other considered parameters must be chosen. OpenSees then runs transient analysis for each of the selected parameters and will output any desired value, in terms of ductility the value needed is the maximum roof drift, $\Delta_{\max }$. Using the $\Delta_{\max }$ from transient analysis and the yield displacement, $\cup_{y}$, from pushover, Equation 6 is used to calculate the ductility in the system.

$$
\mu=\frac{\Delta_{\max }}{U_{y}}
$$

A ductility is calculated at every incremental step of the IDA. At the first instance the system ductility is greater than or equal to the target ductility a flag is created. The RFactor for the system is calculated at that step. See Equation 7 below for a mathematical representation.

$$
R=\left.R_{i}\right|_{\mu_{i} \geq \mu_{0}}
$$

At that step the earthquake scaling factor, $\mathrm{SF}_{\mathrm{i}}$, is used to calculate the R-Factor. Further explanation of the scaling factor can be found in Section 3.3.2. All other components of Equation 8 to calculate the R-Factor are external inputs. The mass of the system, $\mathrm{m}$, is determined on the brace and frequency under considered, see Section 2.2.4 for more on mass. $\left(\left.P S a\right|_{T_{n}}\right)_{\text {original record }}$ is the pseudo-spectral acceleration of the original earthquake record at the frequency under consideration. And lastly $V_{y}$ is the base shear at yield from pushover analysis for the given brace, see Section 3.1 for more on pushover analysis.

$$
\left.R\right|_{S F=S F_{i}}=\frac{m *\left(\left.P S a\right|_{T_{n}}\right)_{\text {original record }} * S F_{i}}{V_{y}}
$$




\subsubsection{Earthquake Suite}

The original ground motion records used are a set of SAC earthquakes recommended for design with a $2 \%$ probability of exceedance in 50 years in the Los Angeles area.

Ground motions details are given in the table below, the motions can be downloaded from the Berkeley strong ground motion website. This suite of earthquakes were chosen because they are relatively large earthquakes so scaling efforts are reduced. Also, this list contains several commonly used earthquakes for research and analysis. Refer to Appendix A for the suite response spectra. [SAC 2011]

Table 3-3: SAC LA 2\% probability of exceedance in 50 years earthquake suite.

\begin{tabular}{|c|c|c|c|c|c|c|c|c|}
\hline $\begin{array}{l}\text { SAC } \\
\text { Name }\end{array}$ & Record & \begin{tabular}{|l} 
Earthquake \\
Magnitude
\end{tabular} & $\begin{array}{c}\text { Distance } \\
(\mathrm{km})\end{array}$ & $\begin{array}{l}\text { Scale } \\
\text { Factor }\end{array}$ & $\begin{array}{l}\text { Number } \\
\text { of Points }\end{array}$ & $\begin{array}{c}\text { DT } \\
(\mathrm{sec})\end{array}$ & $\begin{array}{c}\text { Duration } \\
\text { (sec) }\end{array}$ & $\begin{array}{c}\text { PGA } \\
\left(\mathrm{cm} / \mathrm{sec}^{2}\right)\end{array}$ \\
\hline$\underline{\mathrm{LA} 21}$ & 1995 Kobe & 6.9 & 3.4 & 1.15 & 3000 & 0.02 & 59.98 & 1258.00 \\
\hline$\underline{\mathrm{LA} 22}$ & 1995 Kobe & 6.9 & 3.4 & 1.15 & 3000 & 0.02 & 59.98 & 902.75 \\
\hline$\underline{\mathrm{LA} 23}$ & 1989 Loma Prieta & 7 & 3.5 & 0.82 & 2500 & 0.01 & 24.99 & 409.95 \\
\hline$\underline{\mathrm{LA} 24}$ & 1989 Loma Prieta & 7 & 3.5 & 0.82 & 2500 & 0.01 & 24.99 & 463.76 \\
\hline$\underline{\mathrm{LA} 25}$ & 1994 Northridge & 6.7 & 7.5 & 1.29 & 2990 & 0.005 & 14.945 & 851.62 \\
\hline LA26 & 1994 Northridge & 6.7 & 7.5 & 1.29 & 2990 & 0.005 & 14.945 & 925.29 \\
\hline LA27 & 1994 Northridge & 6.7 & 6.4 & 1.61 & 3000 & 0.02 & 59.98 & 908.70 \\
\hline$\underline{\mathrm{LA} 28}$ & 1994 Northridge & 6.7 & 6.4 & 1.61 & 3000 & 0.02 & 59.98 & 1304.10 \\
\hline$\underline{\mathrm{LA} 29}$ & 1974 Tabas & 7.4 & 1.2 & 1.08 & 2500 & 0.02 & 49.98 & 793.45 \\
\hline$\underline{\mathrm{LA} 30}$ & 1974 Tabas & 7.4 & 1.2 & 1.08 & 2500 & 0.02 & 49.98 & 972.58 \\
\hline$\underline{\mathrm{LA} 31}$ & Elysian Park (simulated) & 7.1 & 17.5 & 1.43 & 3000 & 0.01 & 29.99 & 1271.20 \\
\hline$\underline{\mathrm{LA} 32}$ & Elysian Park (simulated) & 7.1 & 17.5 & 1.43 & 3000 & 0.01 & 29.99 & 1163.50 \\
\hline$\underline{\mathrm{LA} 33}$ & Elysian Park (simulated) & 7.1 & 10.7 & 0.97 & 3000 & 0.01 & 29.99 & 767.26 \\
\hline$\underline{\mathrm{LA} 34}$ & Elysian Park (simulated) & 7.1 & 10.7 & 0.97 & 3000 & 0.01 & 29.99 & 667.59 \\
\hline$\underline{\mathrm{LA} 35}$ & Elysian Park (simulated) & 7.1 & 11.2 & 1.1 & 3000 & 0.01 & 29.99 & 973.16 \\
\hline$\underline{\mathrm{LA} 36}$ & Elysian Park (simulated) & 7.1 & 11.2 & 1.1 & 3000 & 0.01 & 29.99 & 1079.30 \\
\hline$\underline{\mathrm{LA} 37}$ & Palos Verdes (simulated) & 7.1 & 1.5 & 0.9 & 3000 & 0.02 & 59.98 & 697.84 \\
\hline$\underline{\mathrm{LA} 38}$ & Palos Verdes (simulated) & 7.1 & 1.5 & 0.9 & 3000 & 0.02 & 59.98 & 761.31 \\
\hline$\underline{\underline{L A 39}}$ & Palos Verdes (simulated) & 7.1 & 1.5 & 0.88 & 3000 & 0.02 & 59.98 & 490.58 \\
\hline$\underline{\mathrm{LA} 40}$ & Palos Verdes (simulated) & 7.1 & 1.5 & 0.88 & 3000 & 0.02 & 59.98 & 613.28 \\
\hline
\end{tabular}




\subsubsection{Earthquake Scaling Factor}

With the target ductility set to 6 there was no need to waste computation effort in the elastic response region. An initial scaling factor is created to guarantee an inelastic response (e.g. $\mu \geq 1$ ) to start off the analysis of an inputted ground motion. The initial scaling factor is calculated from the displacement where yield occurs during pushover, $U_{y}$, over the spectral displacement, $S_{d}$, as show in Equation 9 below.

$$
S F_{o}=\frac{U_{y}}{S_{d}}
$$

Spectral displacement was calculated with the pseudo-spectral acceleration, PSa, at a given natural period, $\mathrm{T}$, as shown in Equation 10 below.

$$
S_{d}=\frac{P S a}{\left(\frac{2 \pi}{T}\right)^{2}}
$$

After the initial scaling factor is calculated, the earthquake's amplification is increased at a given scaling factor increment to get the response for desired ductility demands. At the start of the project the scaling factor matrix was calculated using:

$$
S F_{\text {new }}=S F_{\text {old }}+\text { SFincrement }
$$

However this proved problematic as initial scaling factors for periods less than 0.2 seconds were often much greater than one. Therefore small incremental steps had little effect on the amplifying earthquake, resulting in accurate values but required many steps during analysis. The opposite is true for the larger periods with initial scaling factors typically much less than one. Where an incremental step of 0.02 could more than double the initial scaling factor, this often skipped over several correct solutions because the step was not well enough refined (e.g. Resurrection). The change in Initial scaling factor versus natural period can be seen in Figure 3.7 below. 


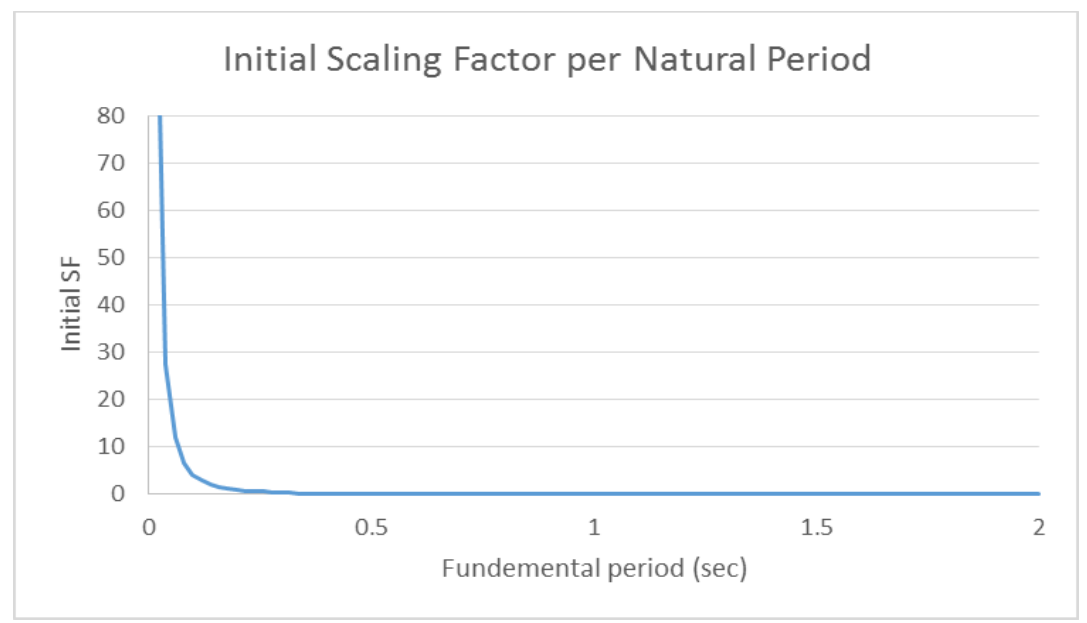

Figure 3.7: Initial scaling factor.

Once this discrepancy was noticed, the method for stepping up the scaling factor was redefined to a percent increase as show in Equation 12 below.

$$
S F_{\text {new }}=S F_{\text {old }}+S \text { Fincrement } * S F_{\text {old }}
$$

The new method for stepping up the scaling factor performed more consistently over the range of periods than the old method, which can be seen in Figure 3.8 below.

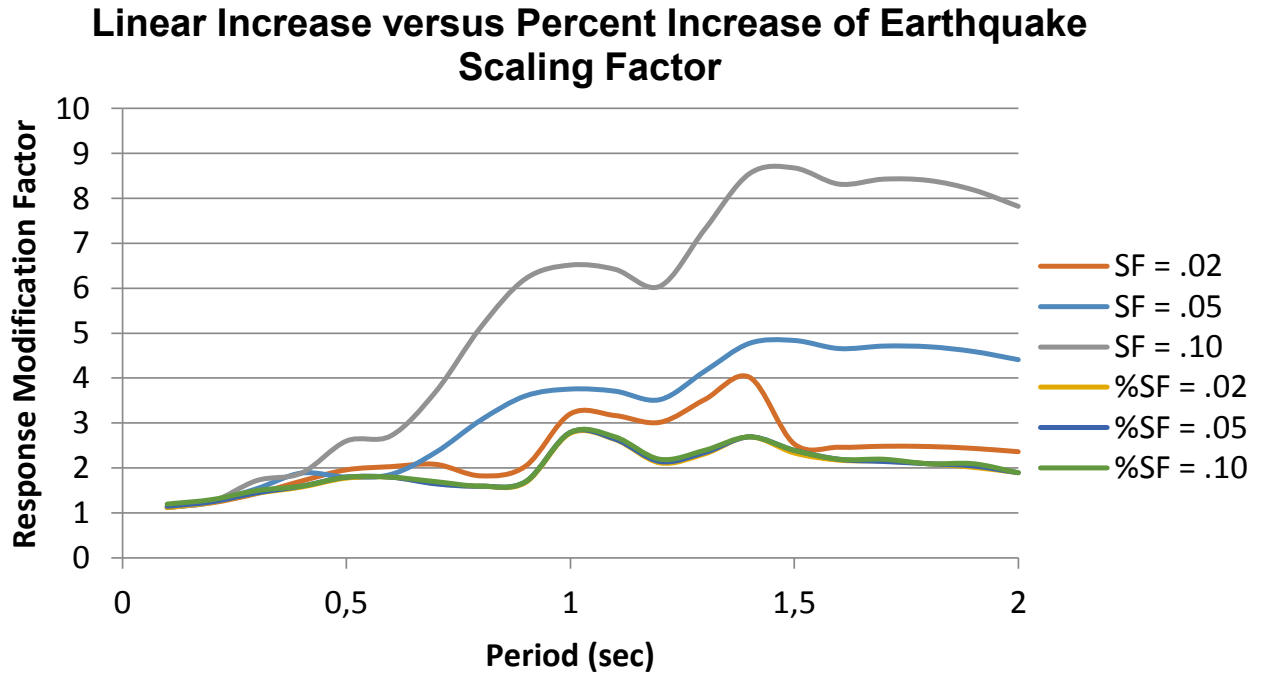

Figure 3.8: Response for EQ 1. Where $S F=$ a linear increase $\% S F=$ a percent increase. 
With a consistent and accurate method to generate the scaling factors the next step is to choose the resolution of the analysis, the incremental step size. The two main considerations for resolution were error due to Resurrection and computational cost. In non-elastic analysis there are often multiple possible solutions, with the first possible solution typically being the most correct. In IDA, if the resolution isn't high enough there is a chance of skipping over the first possible solution. The solver will pick the next viable solution which will most likely not be the most correct solution. This phenomenon is called Resurrection an example can be seen in Figure 3.9 shown below.

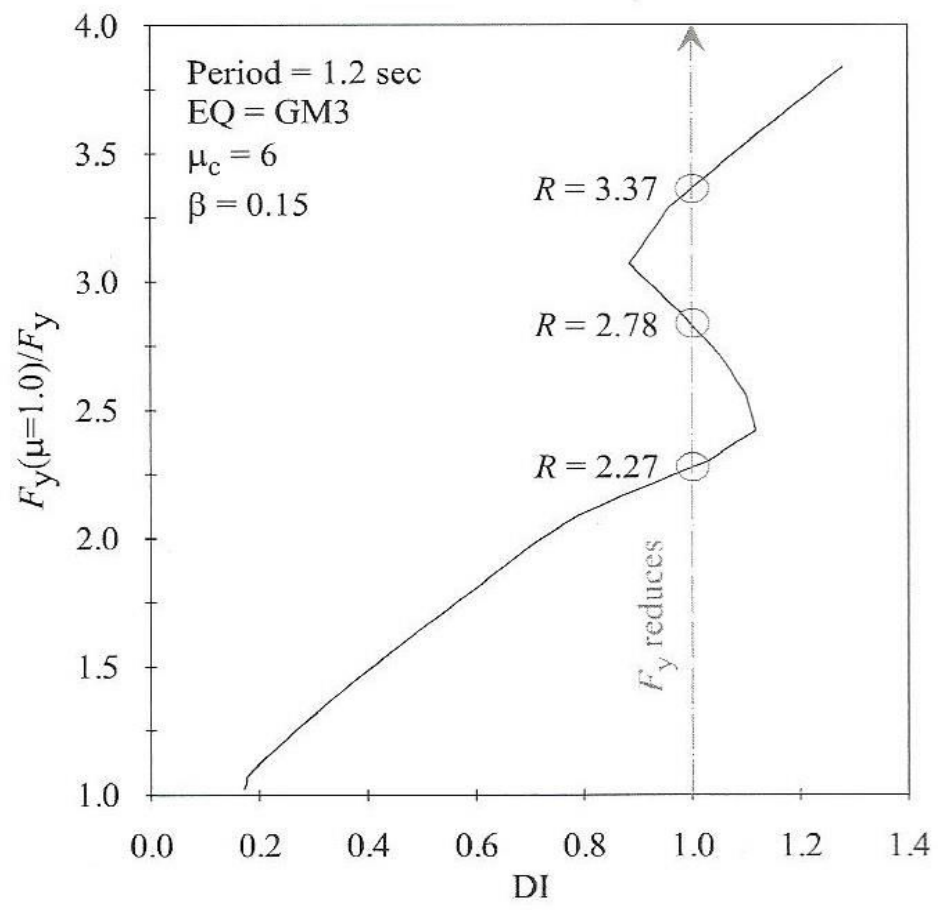

Figure 3.9: Effect of structural resurrection on determination of the $R$ factor [from Hou and Qu 2014].

Several parameter studies were performed to attempt to get an ideal scaling factor increment. A range of $1 \%$ to $25 \%$ increase of the previous step were considered during the studies. As stated previously, the target ductility is set to 6 so the best increment was chosen to best suite that ductility. In looking at the effects of the different scaling factor 
increment had over several braces and several earthquakes it was determined that and increment of 0.02 (or a $2 \%$ increase) was sufficiently accurate. Figure 3.10 shows an example of the effect the scaling factor increment has on the R-Factor for a particular brace and earthquake. Figure 3.11 shows the number of incremental steps required to get to the required ductility for each increment and frequency in Figure 3.10.

\section{Scaling Factor Increment Values for $\boldsymbol{\mu}=6$}

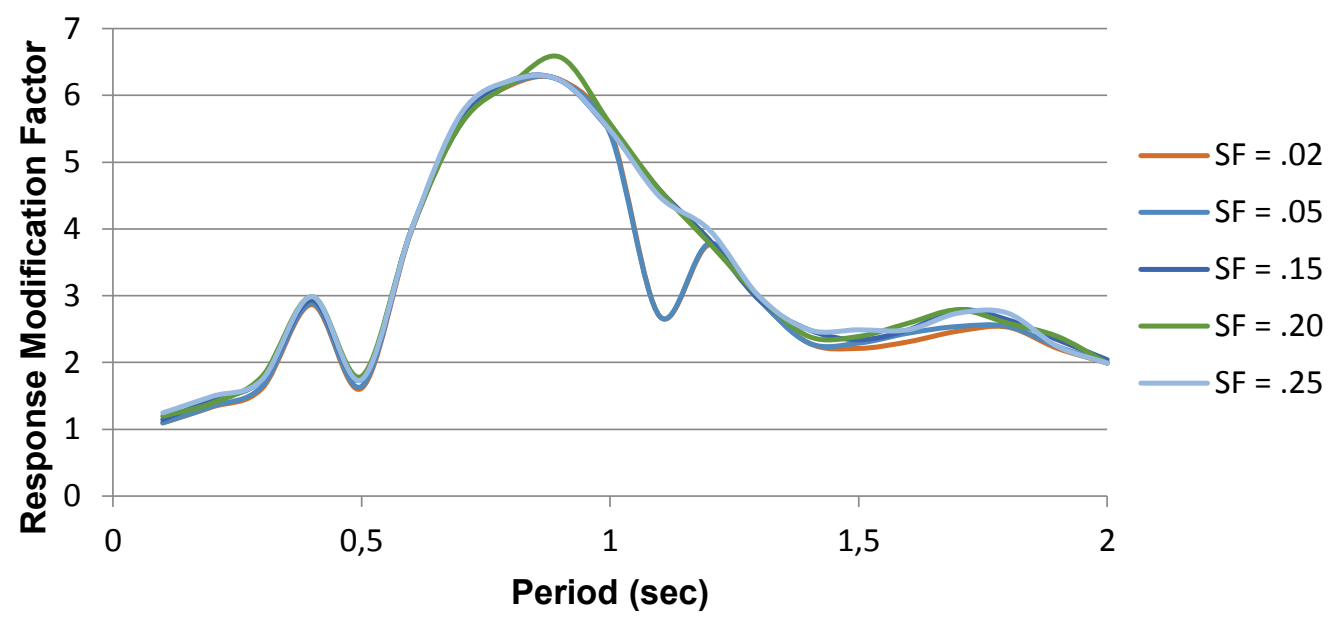

Figure 3.10: Response of Brace 1 from EQ 2.

\section{Relative Computational Time to Acheive $\mu=6$}

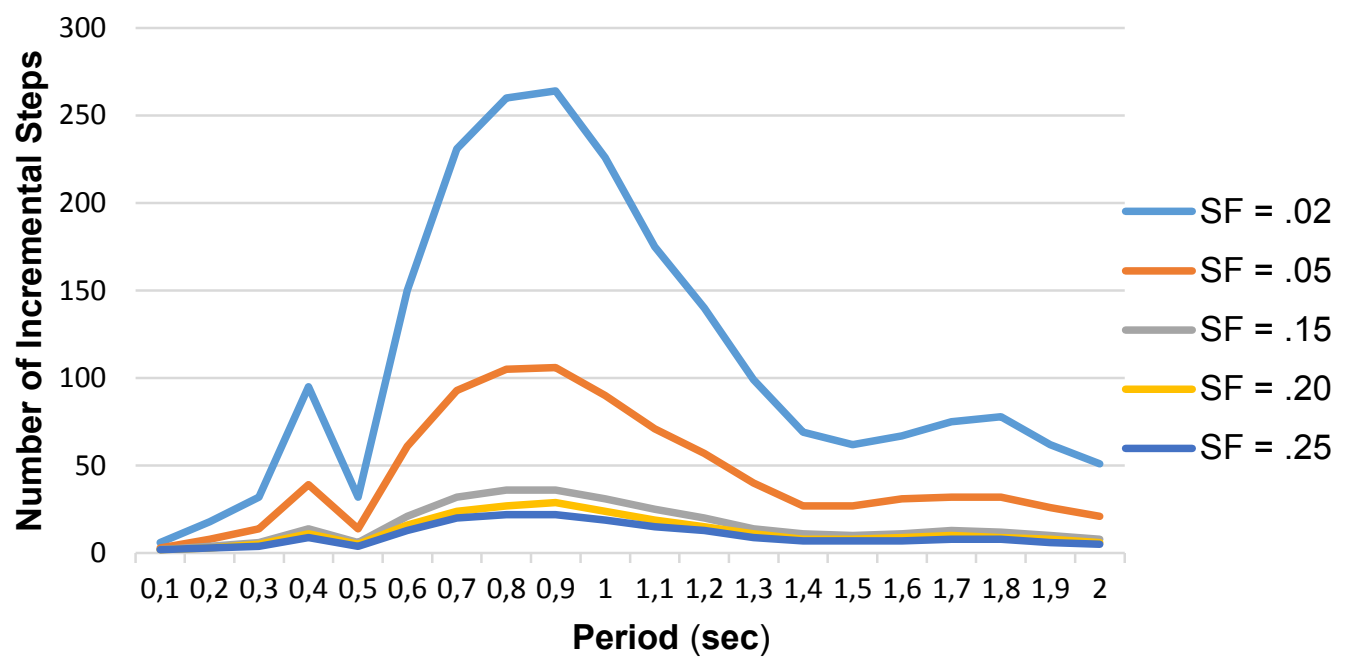

Figure 3.11: Response of Brace 1 from EQ 2. 


\subsection{Geometric Nonlinearities}

In order to calculate global displacements, first local member displacements must be considered. Member displacements are calculated with matrix analysis of member stiffness and loads. The analysis performed in this study is capturing plastic behavior, therefore the stiffness matrix used should include more than just the elastic stiffness. Note that the elastic stiffness matrix can be solely used calculate displacements but there is a chance the algorithms used won't converge to the most correct answer, also this method is less efficient at converging to a solution in term of computational efforts. Newton-Raphson Iteration and Newton-Raphson Iteration with Line Search are the two convergence algorithms used in the transient analysis portion of this thesis. Both of these methods update the stiffness matrix using the unbalanced nodal force from the resisting force calculation.

To improve accuracy and convergence efficiency the matrix for member forces, $r$, is broken into sway, $r_{s}$ and no sway, $r_{n s}$ forces. This can be seen in Equation 13 listed below. The physical interpretation of $r$ can be seen in Figure 3.12 and Figure 3.13 as respective the elements axial force, shear force, and moments.

$$
r=k d=r_{n s}+r_{s}
$$

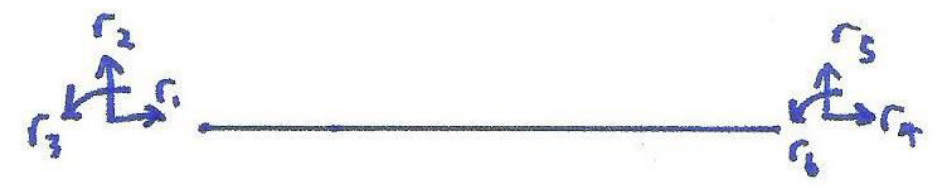

Figure 3.12: Directional assignments of forces in reference system.

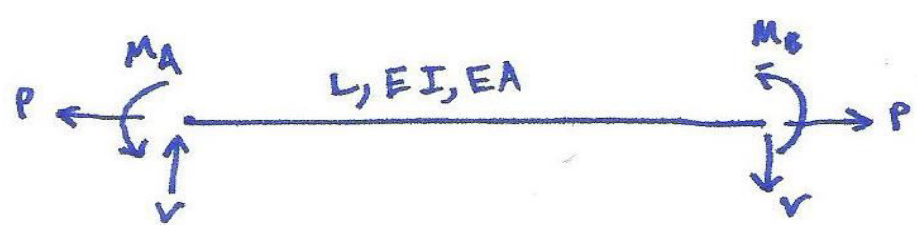

Figure 3.13: Undeformed beam-column. 
First the no sway condition will be considered, this takes P- $\delta$ effects into consideration. The full-blown method of calculating P-ס effects would be with the method provided below.

$$
r_{n s}=\left[\begin{array}{c}
-P \\
V \\
M_{A} \\
P \\
-V \\
M_{B}
\end{array}\right]
$$

Where

$$
V=\frac{M_{A}+M_{B}}{L}
$$

$$
\begin{gathered}
M_{A}=\frac{E I}{L}\left(s_{i i} \theta_{A}+s_{i j} \theta_{B}-\left(s_{i i}+s_{i j}\right) \frac{\Delta}{L}\right)+F E M_{A} \\
M_{B}=\frac{E I}{L}\left(s_{i i} \theta_{B}+s_{i j} \theta_{A}-\left(s_{i i}+s_{i j}\right) \frac{\Delta}{L}\right)+F E M_{B} \\
s_{i i}=s_{j j}=\left[\frac{k L \sin k L-(k L)^{2} \cos k L}{2-2 \cos k L-k L \sin k L}\right] \\
s_{i j}=s_{j i}=\left[\frac{(k L)^{2}-k L \sin k L}{2-2 \cos k L-k L \sin k L}\right]
\end{gathered}
$$

These are several things to note in this method provided. First, the $s_{i i}$ and $s_{i j}$ equations presented are only for members in compression, members in tension have different equations. Also, the $s_{i i}$ and $s_{i j}$ equations do not include shear deformations. The moment equation above includes $\mathrm{P}-\Delta$ effects so to solely consider $\mathrm{P}-\delta, \Delta$ must be set to zero. Finally, this method uses a virtual work assumption that the system is at equilibrium therefore with small displacements the sum of the work is equal to zero. [Archer 2015] 
The braces in this thesis are broken into several imperfect two dimensional trusses. This simplifies no sway force matrix substantially as moments and shears at the end nodes are equal to zero. The simplified force matrix is show below along with the corresponding stiffness matrix.

$$
r_{n s}=\left[\begin{array}{c}
-P \\
0 \\
0 \\
P \\
0 \\
0
\end{array}\right]=\frac{A E}{L}\left[\begin{array}{cccccc}
1 & 0 & 0 & -1 & 0 & 0 \\
0 & 0 & 0 & 0 & 0 & 0 \\
0 & 0 & 0 & 0 & 0 & 0 \\
-1 & 0 & 0 & 1 & 0 & 0 \\
0 & 0 & 0 & 0 & 0 & 0 \\
0 & 0 & 0 & 0 & 0 & 0
\end{array}\right]\left[\begin{array}{l}
d_{1} \\
d_{2} \\
d_{3} \\
d_{4} \\
d_{5} \\
d_{6}
\end{array}\right]
$$

Surprisingly OpenSees does not have any pre-coded protocol to capture P-ठ effects. According to OpenSees [P-delta and corotational transformation] the only way to consider $\mathrm{P}-\delta$ is to break the element under consideration up into several elements and use the protocol for P- $\Delta$. Since the model is broken into trusses there should be no P- $\delta$ effects as every member is therefore a truss. However, since an initial camber was introduced to the braces there will be a pseudo-P- $\delta$ effect created from the P- $\Delta$ protocol over the split brace. The pseudo-P- $\delta$ effect needs to be considered because without it analysis would assume a first order approximation where sii and sij would equal four and two, respectively. This first order approximation is invalid since axial load exists in the members.

From OpenSees, "If you write the equilibrium equations in the deformed configuration $\left(p=b^{*} q\right)$ in the exact form then $\mathrm{kg}=(\text { derivative of } b \text { over displacement field })^{*} q$ is corotational geometric stiffness matrix" where $p$ is the vector of external forces in local coordinate system acting on undeformed element configuration and $q$ is the vector of basic forces of a deformed element [P-delta and corotational transformation]. Since the braces are defined as corotational elements then the geometric stiffness matrix should 
be calculated in the deformed configuration and therefore take into consideration the stiffness matrix for sway and the pseudo no sway.

Formulations for P- $\Delta$ effects are considered in the deformed configuration. Refer to Figure 3.14 below for definitions of latter equations.

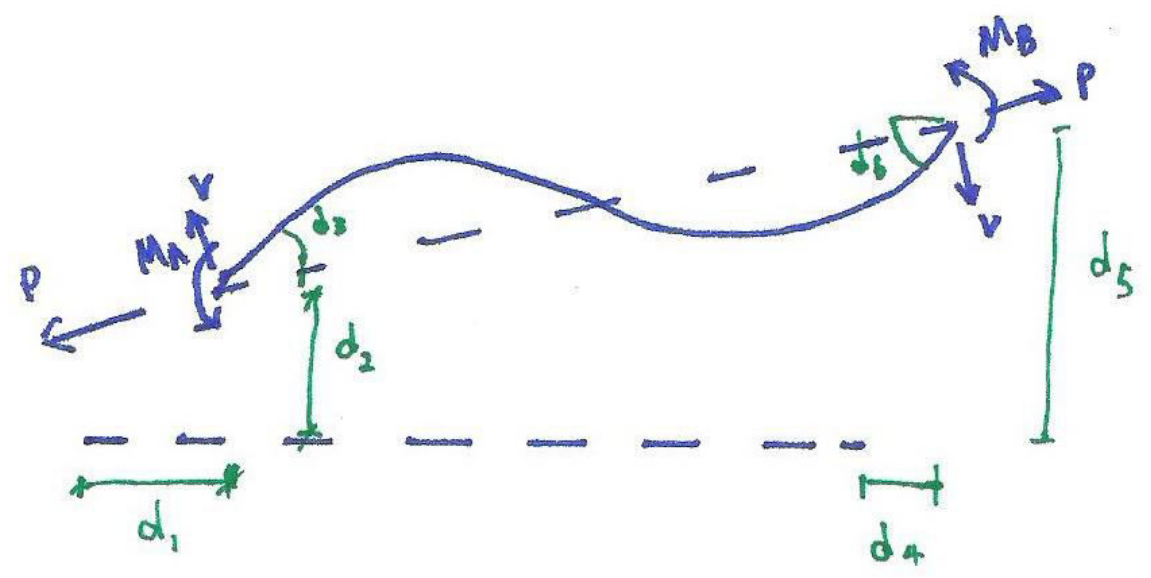

Figure 3.14: Deformed beam-column.

Shear in the deformed configuration, $\mathrm{V}_{\mathrm{NL}}$, is defined in below based on geometry given in Figure 3.14 above.

$$
\begin{gathered}
V_{N L} \approx \frac{M_{A}+M_{B}}{L+\left(d_{4}-d_{1}\right)} \\
\text { chord angle }=\frac{d_{5}-d_{2}}{L}
\end{gathered}
$$

Using the same coordinate system (Figure 3.14), the local matrix for member forces, $r$, can be redefined in the deformed configuration. Note this formulation uses small angle approximation. The new form of member forces can be seen below.

$$
r=\left[\begin{array}{c}
-P-V_{N L}\left(d_{5}-d_{2}\right) / L \\
-P\left(d_{5}-d_{2}\right) / L+V_{N L} \\
M_{A} \\
P+V_{N L}\left(d_{5}-d_{2}\right) / L \\
P\left(d_{5}-d_{2}\right) / L-V_{N L} \\
M_{B}
\end{array}\right]
$$


Member induced by sway can now be calculated by differentiating $r$ in the deformed configuration from $r_{n s}$ in the reference configuration as show in Equation 14 below.

$$
\begin{gathered}
r_{s}=r-r_{n s} \\
r_{S}=\left[\begin{array}{c}
-V_{N L}\left(d_{5}-d_{2}\right) / L \\
-P\left(d_{5}-d_{2}\right) / L+V_{N L}-V \\
0 \\
V_{N L}\left(d_{5}-d_{2}\right) / L \\
P\left(d_{5}-d_{2}\right) / L-V_{N L}+V \\
0
\end{array}\right]
\end{gathered}
$$

$\mathrm{V}_{\mathrm{NL}}$ can be redefined using a Taylor series approximation as shown below. [Archer 2015]

$$
\begin{aligned}
& V_{N L}=\frac{M_{A}+M_{B}}{L}\left(\frac{1}{1+\frac{d_{4}-d_{1}}{L}}\right) \approx \frac{M_{A}+M_{B}}{L}\left(1-\frac{d_{4}-d_{1}}{L}\right) \\
& \therefore V_{N L}-V=-\frac{M_{A}+M_{B}}{L}\left(\frac{d_{4}-d_{1}}{L}\right)=-V\left(\frac{d_{4}-d_{1}}{L}\right) \\
& \therefore r_{s}=\left[\begin{array}{c}
-V_{N L}\left(d_{5}-d_{2}\right) / L \\
-P\left(d_{5}-d_{2}\right) / L-V\left(d_{4}-d_{1}\right) / L \\
0 \\
V_{N L}\left(d_{5}-d_{2}\right) / L \\
P\left(d_{5}-d_{2}\right) / L+V\left(d_{4}-d_{1}\right) / L \\
0
\end{array}\right] \\
& {\left[\begin{array}{l}
r_{1} \\
r_{2} \\
r_{3} \\
r_{4} \\
r_{5} \\
r_{6}
\end{array}\right]=\left[\begin{array}{cccccc}
0 & V_{N L} / L & 0 & 0 & -V_{N L} / L & 0 \\
V / L & P / L & 0 & -V / L & -P / L & 0 \\
0 & 0 & 0 & 0 & 0 & 0 \\
0 & -V_{N L} / L & 0 & 0 & V_{N L} / L & 0 \\
-V / L & -P / L & 0 & V / L & P / L & 0 \\
0 & 0 & & 0 & 0 & 0
\end{array}\right]\left[\begin{array}{l}
d_{1} \\
d_{2} \\
d_{3} \\
d_{4} \\
d_{5} \\
d_{6}
\end{array}\right]}
\end{aligned}
$$

OpenSees' pre-coded protocols used do have the capability to use the correct stiffness matrix for sway (e.g. geometric stiffness). Shear terms are typically disregarded in most computation programs which is not mathematically correct. However, as previously 58 
stated, the members used are trusses so for analysis the shear terms are disregarded. A case study is conducted to determine the effects of $\mathrm{P}-\delta, \mathrm{P}-\Delta$, and the number of elements that the brace needs to be split up into. Results are shown in Sector 5.1.

The purpose of looking at geometric nonlinearities is to improve the likelihood of converging to the most correct answer while improving computational time. In the OpenSees model in this thesis, the braces use corotational geometric transformation. All other members use P-delta geometric transformation. Dr. Denavit and Dr. Hajjar of Northeastern University did a study on each of the OpenSees geometric transformations. Results of their work, shown in Figure 3.15 below, further support the use of twenty element as sufficient, as discussed in earlier sections. [Denavit and Hajjar 2013]

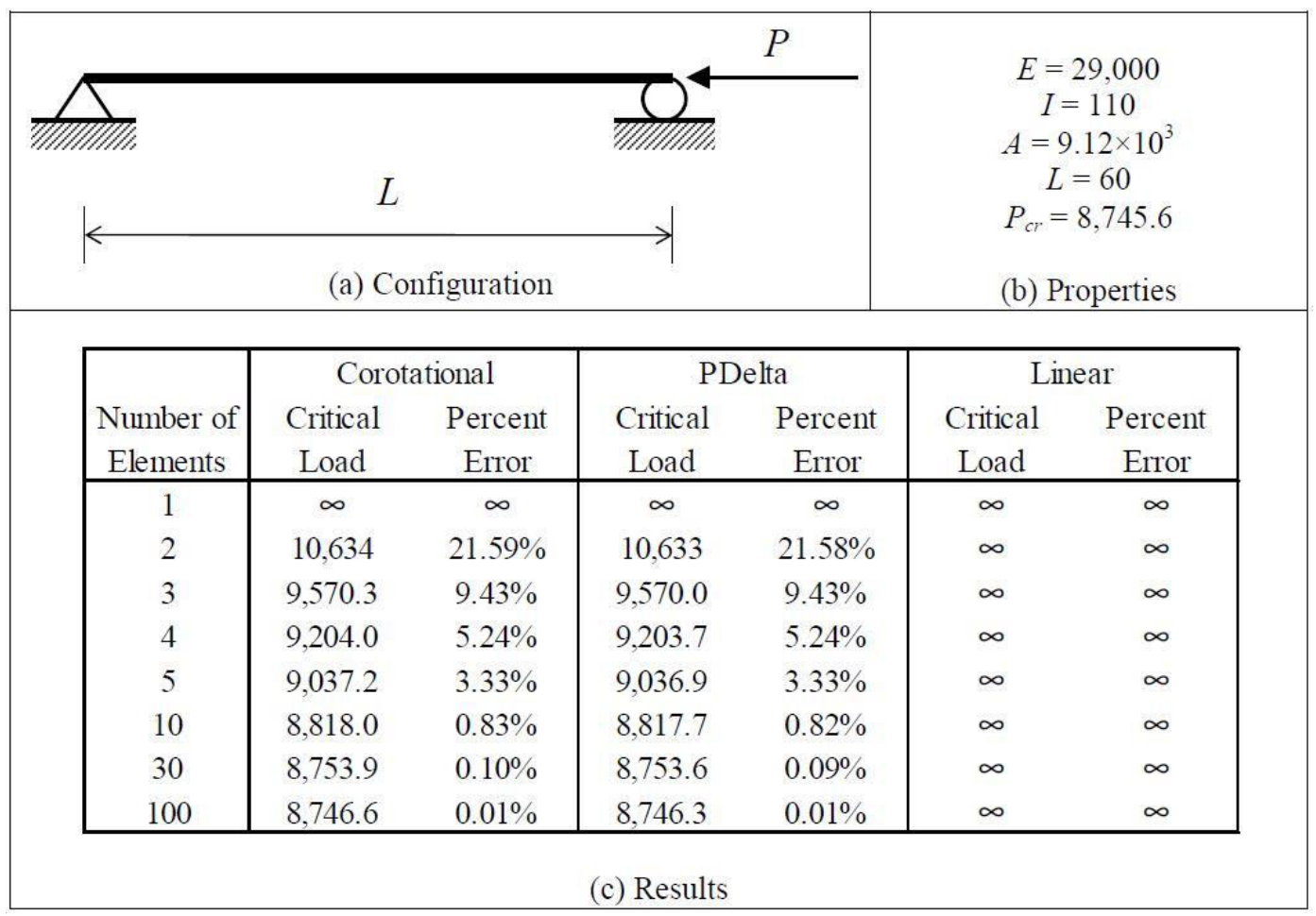

Figure 3.15: Simply supported beam under axial load [from Denavit and Hajjar 2013]. 


\subsection{Material Nonlinearities}

Material Nonlinearities are based on an action-deformation relationship. Their

relationship must be interpreted and calibrated from experimental data as they are physical properties of the material. Isotropic hardening is an example of a material nonlinearity and can be seen post yield in Figure 3.3 of pushover analysis. Steel02 (the material the braces are made of) uses commit routine to update the state of the element. This method is a very expensive protocol because it require several values to be calculated and stored. Each iteration the program must calculate the stress, strain, tangent, and the intersection of the tangent with the strain hardening asymptote and store most of the associated data for the next iteration. Using commit instead of trial lets the material be nonlinear and inelastic instead of just nonlinear and elastic, which can result in a more accurate answer while capturing the true effects in a more realistic manner. This method is preferred since OpenSees is often used as research software but again commit is expensive in terms of computation and memory. Figure 3.16 below helps illustrate the many changes in tangents and movement of the origin. [McKenna and Fenves 2004; Archer 2015]
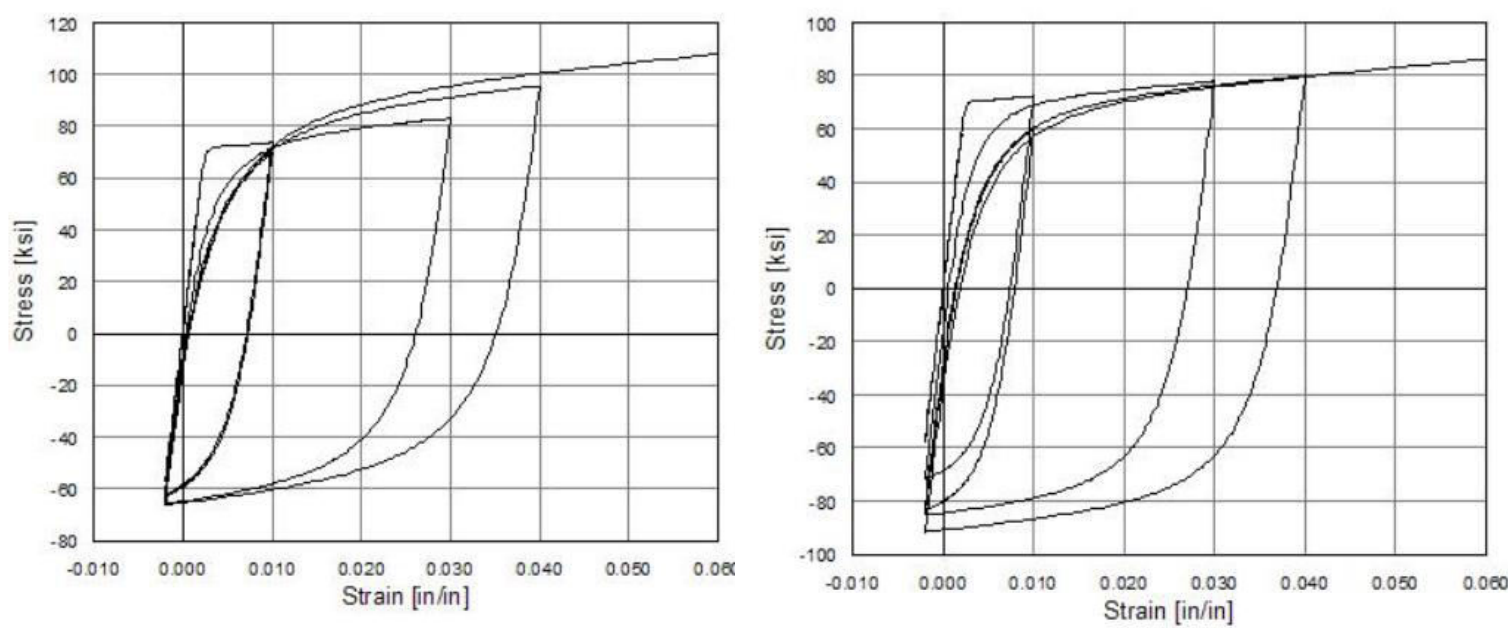

Figure 3.16: Example hysteretic behavior with isotropic hardening in tension and compression, respectively [from McKenna and Fenves 2004]. 
Below is a brief outline of OpenSees' protocol for Steel02 for material nonlinearities:

1. Initializes script

2. Copies current data

3. Alter the copy with a trial run

4. Checks trail update versus predefined parameters

5. Marks values that satisfy criteria with flag

6. Updates state based on flags

As previously stated fatigue material acts as a secondary material to Steel02. The fatigue material follows a similar protocol of commit to that of Steel02. Once the state is updated the program needs to determine the following steps:

1. Check if the parent material has failed, there is no point of continuing analysis if true

2. Check if element has surpassed predetermined strains/deformations

3. Check if at peak (if not pretend that program is)

4. Find slope, mark second and third peak

5. Check for damage by beginning counter

6. Flag and store necessary data

7. Commit and output response (stress, strain, tangent, and damage)

8. Run case check and update state 
OpenSees must solve the fatigue material and Steel02 simultaneously. Therefore this method of analysis is extremely computationally and memory expensive. However this thesis, and several theses and dissertations before it, is trying to show the importance of low-cycle fatigue so it must be included. Note that this method uses linear accumulation which may not be appropriate for high cycle fatigue but that is not really a concern in this project. The fatigue material will continue to update its state until the damage reaches $100 \%$ as shown in the Figure 3.17 below. After $100 \%$ damage accumulation the element is assumed to have ruptured so that element stiffness is removed from the system.

[McKenna and Fenves 2004; OpenSees Subversion Repositories; Archer 2015]
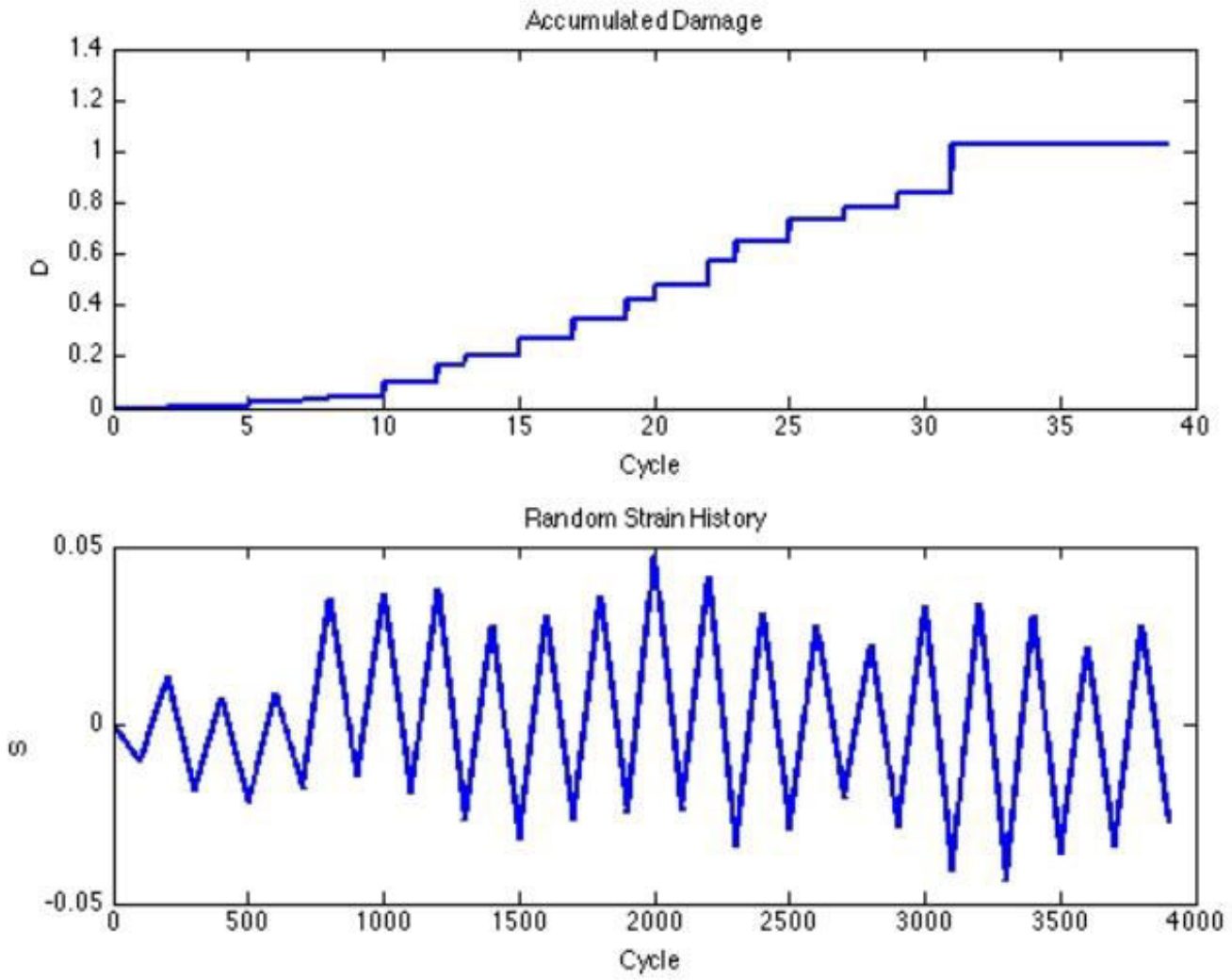

Figure 3.17: Example accumulated damage and strain of a model with arbitrary strain history, respectively. [Images from McKenna and Fenves 2004]. 


\section{Fatigue Model and Failure Criteria}

There are two general approaches for modeling material failure: one at the local scale and the other at the global scale. The local scale approach focuses on issues related to micromechanics, whereas the global scale approach focuses of fracture mechanics [Huang and Mahin 2010]. As stated earlier, the fiber model used is unable to capture local buckling or facture mechanics. However since the sections are compact the effects of local buckling should be lessened. These affects should also be lessened with sufficient calibration with experimental data. Previous studies in wide flange sections [Ballio and Castiglioni 1995; Bertero and Popov 1965; Fisher et al. 1997; Krawinkler et al. 1983; Popov and Pinkey 1969; Stojadinović 2003] have shown large scatter of fatigue life for similar configurations under similar loadings. Therefore results can only be assumed to be reasonably accurate to within a few cycles. [Uriz and Mahin 2008] Considering fatigue life is crucial in accurately determining when failure will occur. Several recent papers have suggested the importance of including fatigue life in structural response models, in particular low-cycle fatigue [Huang and Mahin 2010; Li and Fahnestock 2013; Li and Fahnestock 2013; Uriz and Mahin 2008]. Fatigue life can be classified into two relative categories, high cycle and low-cycle. High cycle fatigue represents plastic strain accumulation due to mid to high intensity loading over several events. Low-cycle fatigue represents plastic strain accumulation due to high intensity loading within a single event. Due to the large computational expense, low-cycle fatigue analysis is typically only considered in hysteretic curves. Damage accumulation due to constant and smooth hysteretic loading is much easier to calculate than damage accumulation due to sporadic seismic events. This thesis concentrates on the effect of low-cycle fatigue has on the seismic response of braces with varying slenderness. 


\subsection{Fatigue Material}

The fatigue material used in this thesis was created and calibrated by Dr. Uriz and Dr.

Mahin in their PEER Report, Toward Earthquake-Resistant Design of Concentrically

Braced Steel-Frame Structures [Uriz and Mahin 2008]. This material was then accepted

by OpenSees and implemented into its material library [McKenna and Fenves 2004].

This section references how the material behaves and incorporates the effects of low-

cycle fatigue.

Since the fiber model is unable to capture fracture mechanics, failure is determined on the local scale with the strain histories. Models of low-cycle fatigue traditionally used a Coffin-Mason relationship: a linear relation between the log of the number of constant amplitude cycle of failure, $N_{f}$, and the log of the strain in each cycle, $\varepsilon_{i}$ [Uriz and Mahin 2008; ASTM 2003; Fisher et al. 1997; Glinka and Kam 1987]. Damage is then approximated by dividing the number of cycles at that amplitude by the number of cycles at that amplitude required for failure. The sum of the damage at a given time, damage index, represent the damage accumulation. A damage index of zero represents virgin material and an index of 1 represents failure. The summation of damage accumulation is adopted from a rainflow accumulation model know as Miner's rule. [Uriz and Mahin 2008; McKenna and Fenves 2004]

Use of the linear Coffin-Mason relationship to capture the effects of low-cycle fatigue has several limitations. One of the main draw backs it that this model applies to strain cycles that are smooth and have consistent amplitudes. Therefore, this model can only capture hysteretic response under a relatively smooth loading, and this model won't be accurate under erratic seismic loading. This model also accumulates damage independent of the 
sequence of the cycles. And finally, the linear relation on the log-log scale generally results in disproportionate damage for large cycles. [Uriz and Mahin 2008]

To remedy some of limitations of the linear Coffin-Mason relationship a modified cycle counting scheme is implemented. The most accurate method is to evaluate the entire strain history to identify and count cycles, but looking at the entire strain history requires a lot of storage. To be more computationally efficient, the modified method used in the fatigue model only keeps track of the four most recent peaks in strain at a given time. Using the last four peaks, an intricate scheme defines the cycles and the associated damage. For a complete discussion, with supporting calculations, of cycle counting and damage index refer to Toward Earthquake-Resistant Design of Concentrically Braced Steel-Frame Structures. Analytical and experimental studies validate the model and calibrate specified input values to account for assumptions and limitations. [Uriz and Mahin 2008; McKenna and Fenves 2004]

As previously stated, this fatigue material has been accepted and implemented in OpenSees' material library. The fatigue material is used in conjunction with a parent material, in this thesis uniaxial Giuffré-Menegotto-Pinto steel with isotropic strain hardening. The fatigue material wraps around each of the fibers of the parent material, while not adding any additional stiffness. The material monitors strains during analysis and accumulates damage. Once the damage accumulation a fiber surpasses unity then fatigue life has been exhausted, the stiffness of parent material for that fiber is set to zero. Refer to Figure 3.17 for visual representation of damage accumulation for a given strain history. Section 3.5 gives a step by step representation of how the fatigue and parent materials behave during excitation. 


\subsection{Definition of Failure}

In FEMA/SAC methodology, failure is defined when the rate of increase of peak

interstory drift with increasing ground motion intensity exceeds five times that associated with an elastic system (e.g. $\mu=5)$ or at a prescribed maximum interstory drift ratio beyond which the reliability of the analysis is considered doubtful (e.g. typically $10 \%$ interstory drift) [SAC 2011; AISC 2005; Uriz and Mahin 2008]. Failure can also be defined rupture in either or both of the braces.

In this thesis, analysis is ran until a ductility greater than or equal to 6 is achieved. This failure criteria is determined from outputted horizontal roof displacements. OpenSees will continue to run even if both braces have ruptured due to the ghost members and boundary members being perfect members. As stated earlier, the materials used do not take into consideration fracture mechanics or local buckling. However, if both braces have ruptured then the system's lateral stiffness will greatly diminish and the ductility should reach 6 quickly.

The effects of post buckling stability are not considered as they are outside of the scope of this thesis. The model used throughout should not have any issues with stability due to the existence of the ghost members. Excluding these members, with the current model, will allow for instability in the system but often leads to crashing of the executable. Changes would have to be made to the model, or create a new model, to analyze the effects of stability. Note that in physical applications post buckling stability may be of major concern and would need to be analyzed. 


\section{Results}

This chapter discusses the results of P-delta and fatigue studies under multiple loading types. Several preliminary parameter studies were need to determine the appropriate inputs in the P-delta and fatigue studies. These preliminary studies include: base shear from monotonic pushover, scaling factor increment selection, subdivision of the brace, and initial imperfection. Full discussion for each of these preliminary studies can be found in Section 3.1, Section 3.3.2, Section 2.2.1.3, and Section 2.2.1.7, respectively.

\subsection{P-delta Effects}

A leaning gravity column is added to the model to represent the presence of multiple gravity frames that the CBF has to support with lateral resistance. To capture the leaning column effect a study was done to capture how the various braces performed against multiple leaning loads. The leaning effect is created with a vertical load applied to the gravity frame, see Figure 2.1. The magnitude of the load is calculated using Equation 3, restated below.

$$
P_{\text {leaning }}=\Delta_{\text {drift }} h K
$$

Where $\Delta_{\text {drift }}$ is roof drift as the dependent variable that varies from 0 to $10 \%, \mathrm{~h}$ is story height, and $\mathrm{K}$ is the lateral stiffness the braces supply to the system [AISC 2005]. The monotonic pushover study uses a $\Delta_{\text {drift }}$ of $0 \%, 2 \%, 5 \%$, and $10 \%$ and the IDA study uses $\Delta_{\text {drift }}$ of $0 \%, 2 \%$, and $10 \%$, values are shown in Table $5-1$ below.

\section{Table 5-1: Leaning column loads (Ibs).}

\section{P-Delta Load}

\begin{tabular}{|r|r|r|r|r|}
\hline \% Drift & Brace & Brace & Brace & Brace \\
& 1 & 2 & 3 & 4 \\
\hline 0 & 0 & 0 & 0 & 0 \\
\hline 2 & 17422 & 7122 & 3245 & 2141 \\
\hline 5 & 43555 & 17804 & 8112 & 5353 \\
\hline 10 & 87110 & 35608 & 16224 & 10706 \\
\hline
\end{tabular}




\subsubsection{Monotonic Pushover}

A P-delta study is conducted with Monotonic Pushover as a means of comparison. In order to avoid convoluting the effects of P-delta, fatigue is not considered in this study.

All other parameters are set to default values recommended/chosen in preliminary studies. Figure 5.1.a to 5.1.d shows the effects the leaning column has on the base shear of the system per each respective brace.

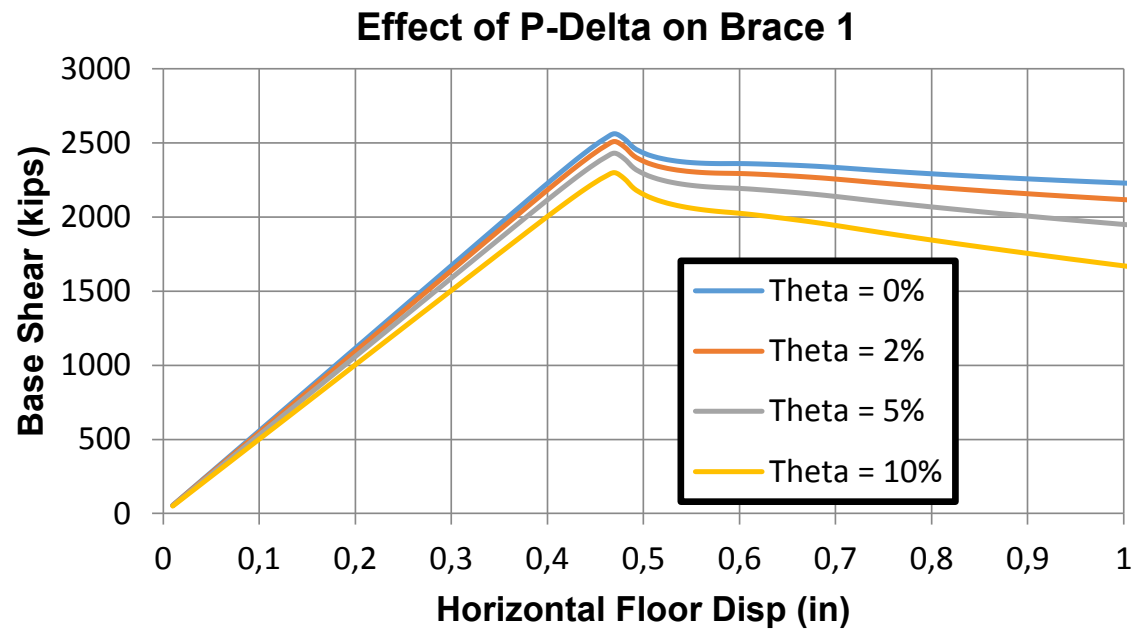

Figure 5.1.a: Leaning column effects on Brace 1 under pushover.

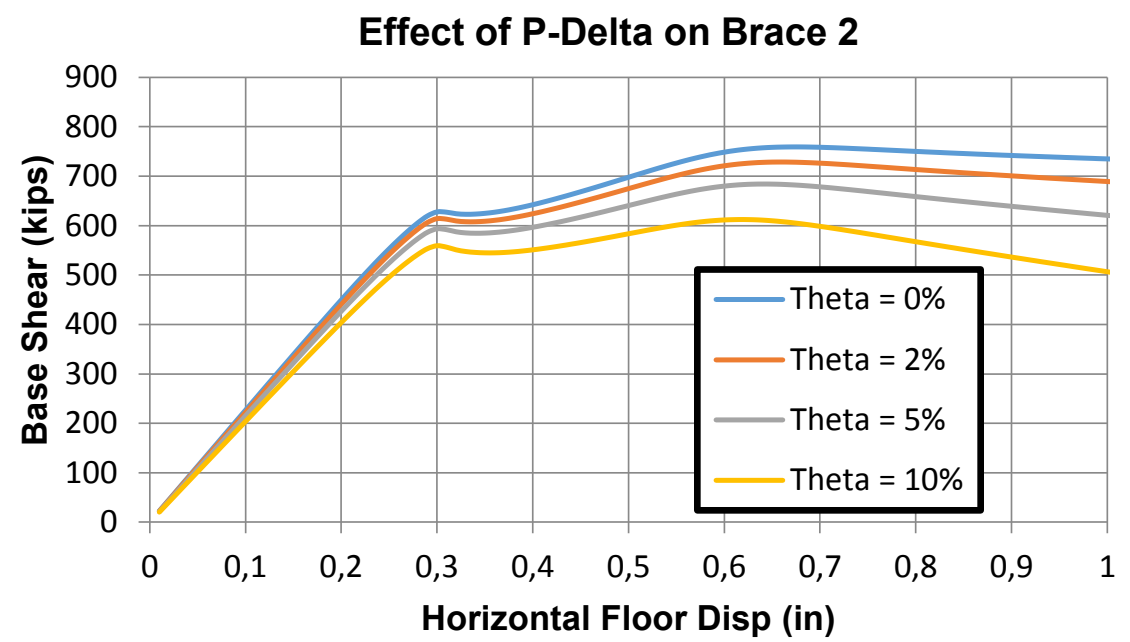

Figure 5.1.b: Leaning column effects on Brace 2 under pushover. 
Effect of P-Delta on Brace 3

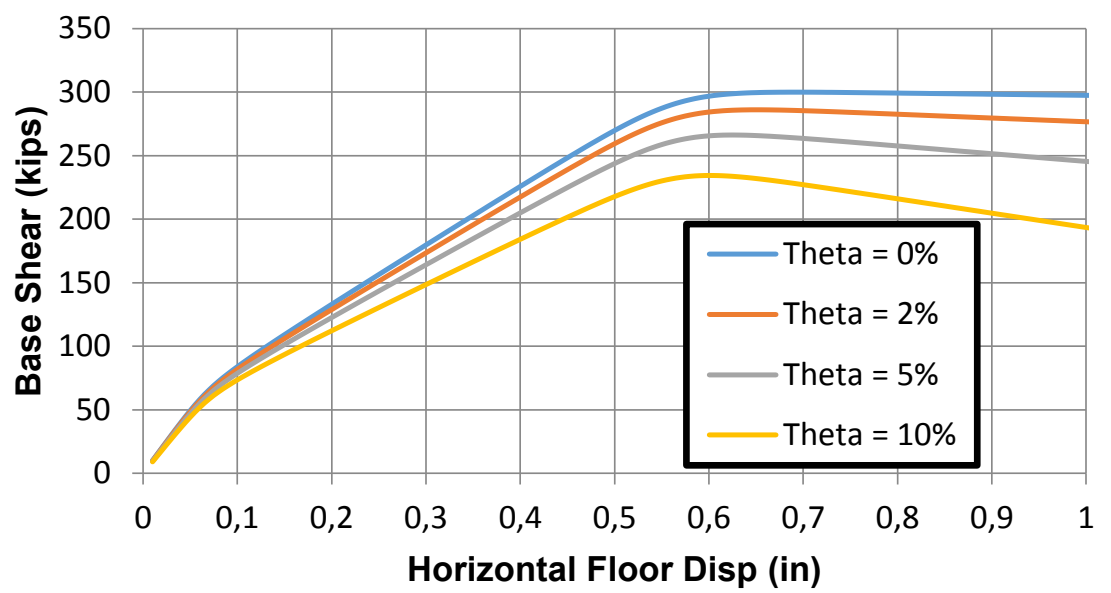

Figure 5.1.c: Leaning column effects on Brace 3 under pushover.

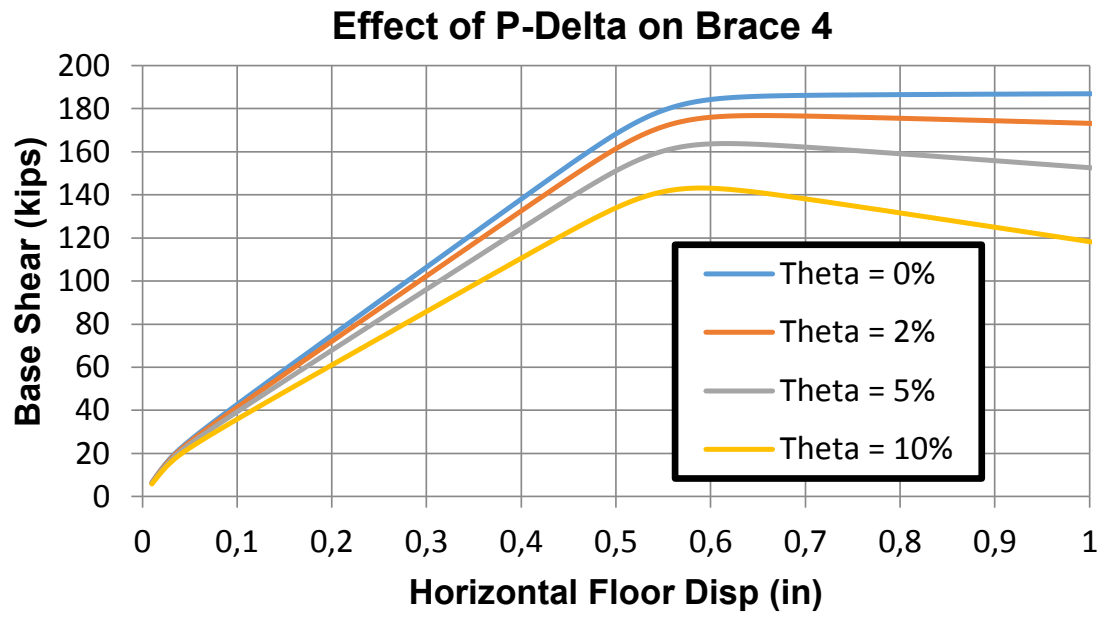

Figure 5.1.d: Leaning column effects on Brace 4 under pushover.

Based on the data collected from monotonic pushover the following trends are observed:

(1) relative reduction in base shears is greater for more slender braces for given drifts, (2) leaning column effects are greater as the section becomes more plastic. These trends seem logical since the force imbalance is greater in more slender braces. And stiffness derogates as the section becomes increasingly plastic, reducing the system's ability to support the leaning column. 


\subsubsection{Incremental Dynamic Analysis}

A full IDA study was done for all 20 earthquakes for the drifts of $0 \%, 2 \%$, and $10 \%$. All comparisons of performance in this section are though the R-factor of the system. The data in this section is presented in several means in order to attempt to gain insight of how the braces behave while supporting leaning column. The first representation of data is shown below in Figure 5.2.a to 5.2.d. This representation shows the reduction in strength of the system for each brace for a single considered earthquake.

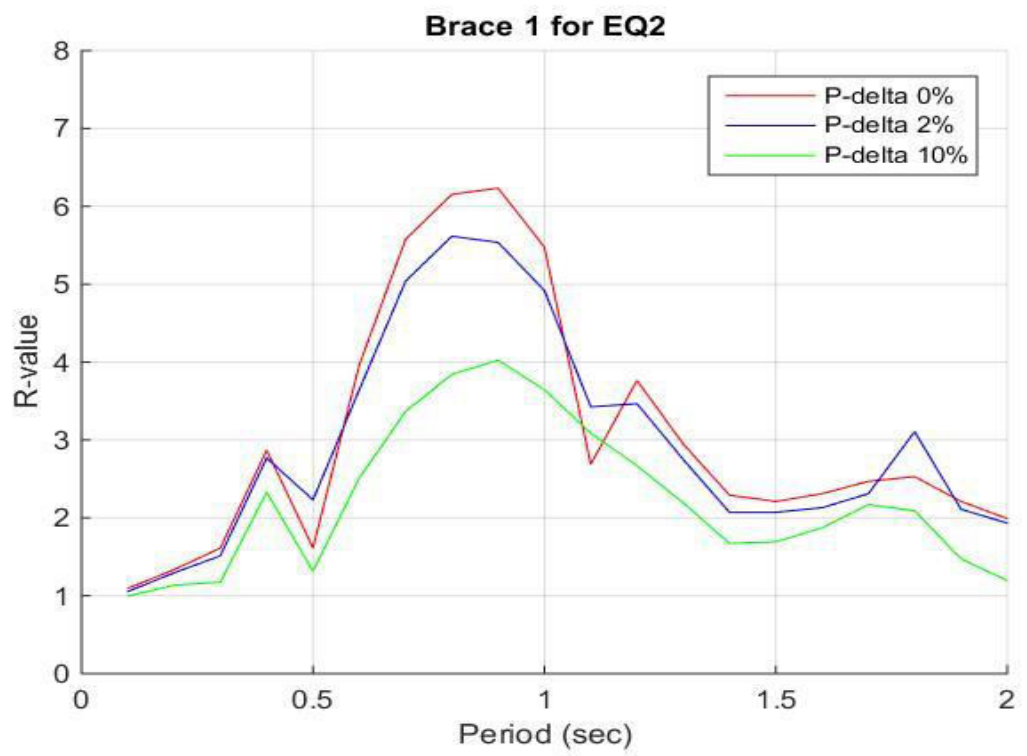

Figure 5.2.a: Leaning column effects on Brace 1 during 1995 Kobe Earthquake. 


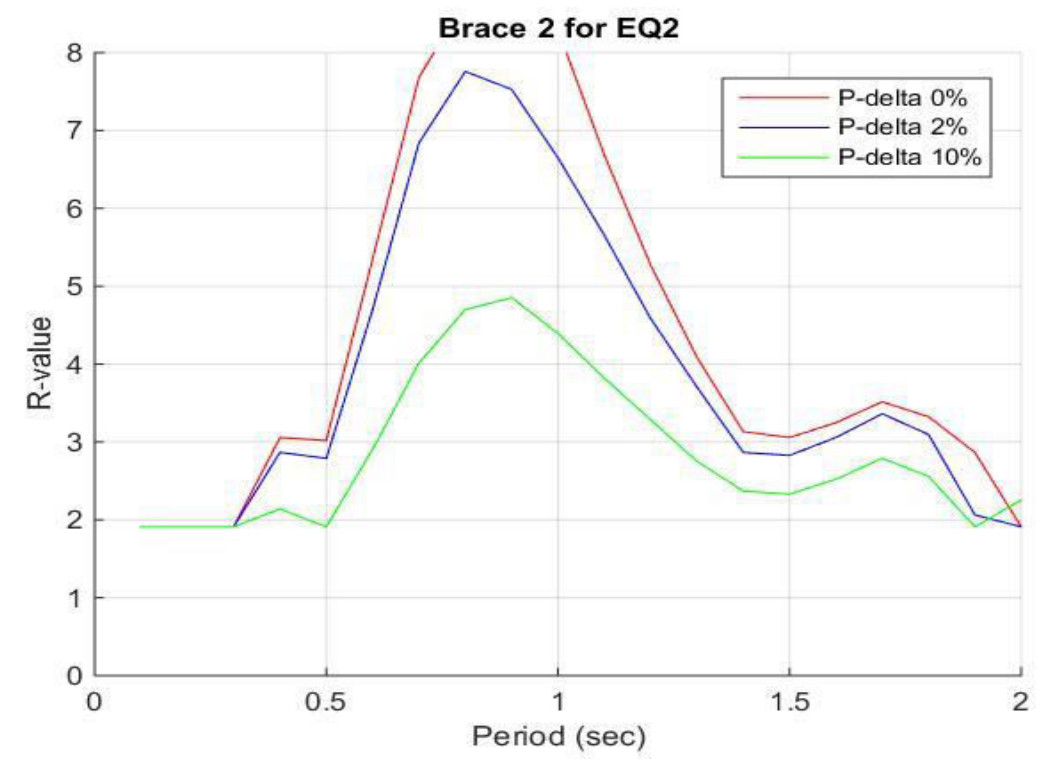

Figure 5.2.b: Leaning column effects on Brace 2 during 1995 Kobe Earthquake.

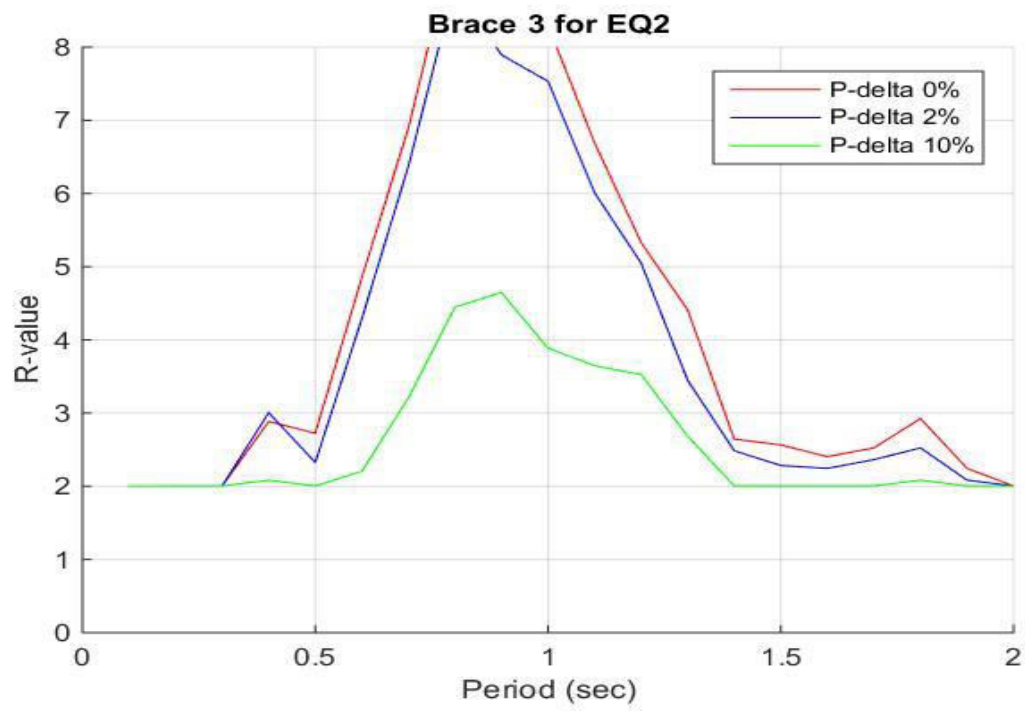

Figure 5.2.c: Leaning column effects on Brace 3 during 1995 Kobe Earthquake. 


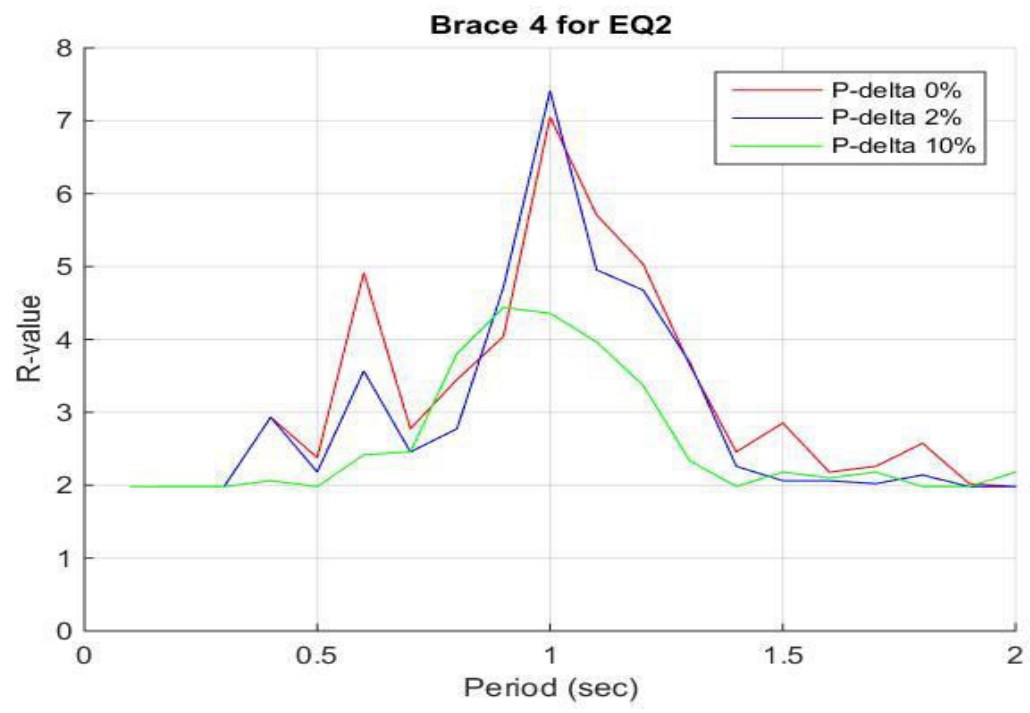

Figure 5.2.d: Leaning column effects on Brace 4 during 1995 Kobe Earthquake.

Based on the data for this particular earthquake (EQ2) the following trends are observed: (1) the R-factor is reduced with an increase in the lean column effect, with some variance, (2) R-factors, and the variance between points at a specific period, are larger for period ranges slightly less than one second, and (3) braces 2, 3, and 4 start at an R-factor of approximately 2 instead of 1 .

Each of these trends can be explained but more analysis is required to state this explinations with an exceptable level of confidence. The first trend is logical because an increase in loading will reduce the systems capactiy and should therefore reduce its ductility capabilities. The second trend where the ductility response of the braces being larger at certain period ranges is due to the seismological properties of the 1995 Kobe Earthquake (EQ2). Analysis done over many earthquakes should diminish this variance. 
The third trend is a phenomina that occurred in every study conducted. The best explanation for this phenomina is due to the large discrepancy bewteen the base shears of the braces. Figure 3.3 shows that the base shear in Brace 1 is almost a full order of magnitude greater than the base shear of Brace 2 and 4 . Using a modified verson of Equation 6 can show the effect of having a small base shears has on ductility. Equation 15.a and 15.b below represents the change in ductility with a target ductility of 1.0 and 6.0 , respectively.

$$
\begin{gathered}
\left.\Delta \mu\right|_{\mu_{\text {target }=1}=\frac{x+\Delta x}{U_{y}}} \\
\left.\Delta \mu\right|_{\mu_{\text {target }=6}=\frac{6 x+\Delta x}{6 U_{y}}=\frac{x}{U_{y}}+\frac{\Delta x}{6 U_{y}}}
\end{gathered}
$$

Where $\mathrm{x}$ is the horizontal displacement at the last point and $\Delta \mathrm{x}$ is the change in displacement in a given incremental step. Note that this effect only seems to happen when calculating the initial scaling factor. Though this does phenomina does make the minimum allowable R-factor for Brace 2, 3, and 4 equal to 2, effects should be minimal in the context of this thesis since the prescribed R-factor in the code is equal to 6 for SCBFs. Any errors in the calculation of Uy may amplify this effect.

The next method of representing the data is shown in Figure 5.3.a to 5.3.d. This representation includes the results of each $\mathrm{P}$-delta over the full earthquake suite and the median of each. 


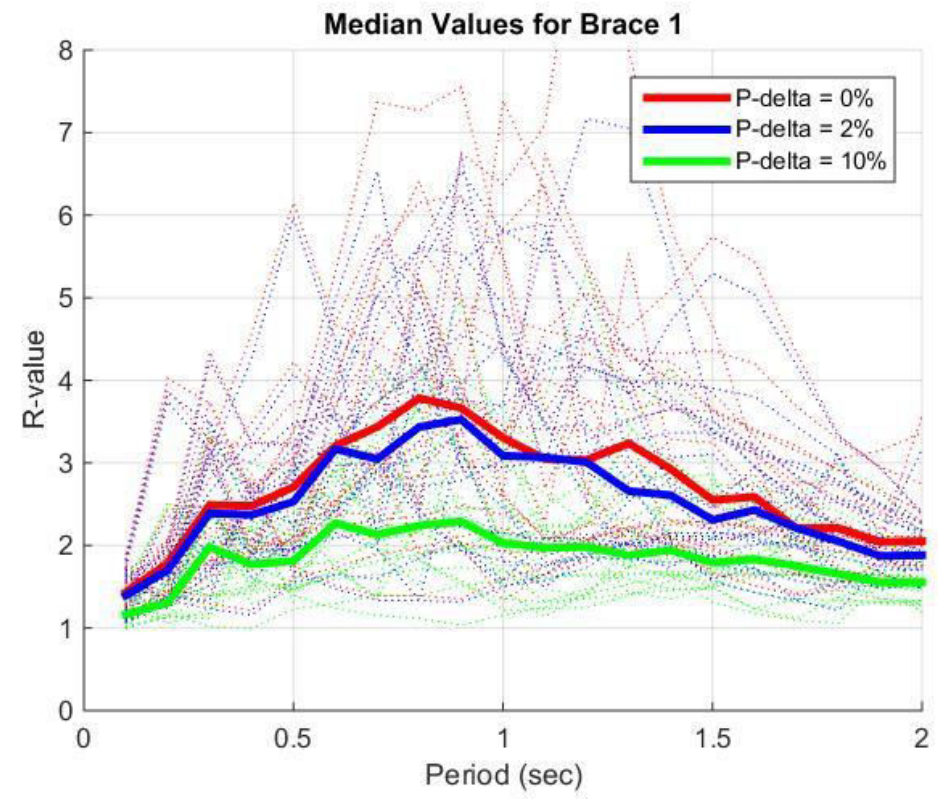

Figure 5.3.a: Median leaning column effects on Brace 1 under the EQ suite.

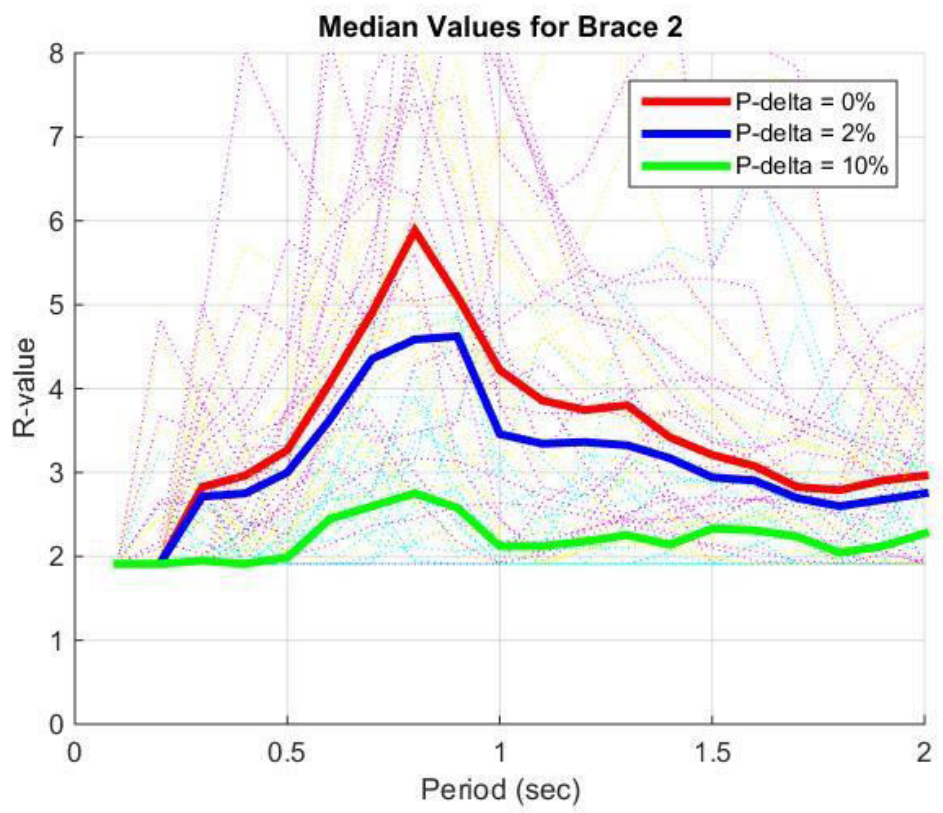

Figure 5.3.b: Median leaning column effects on Brace 2 under the EQ suite. 


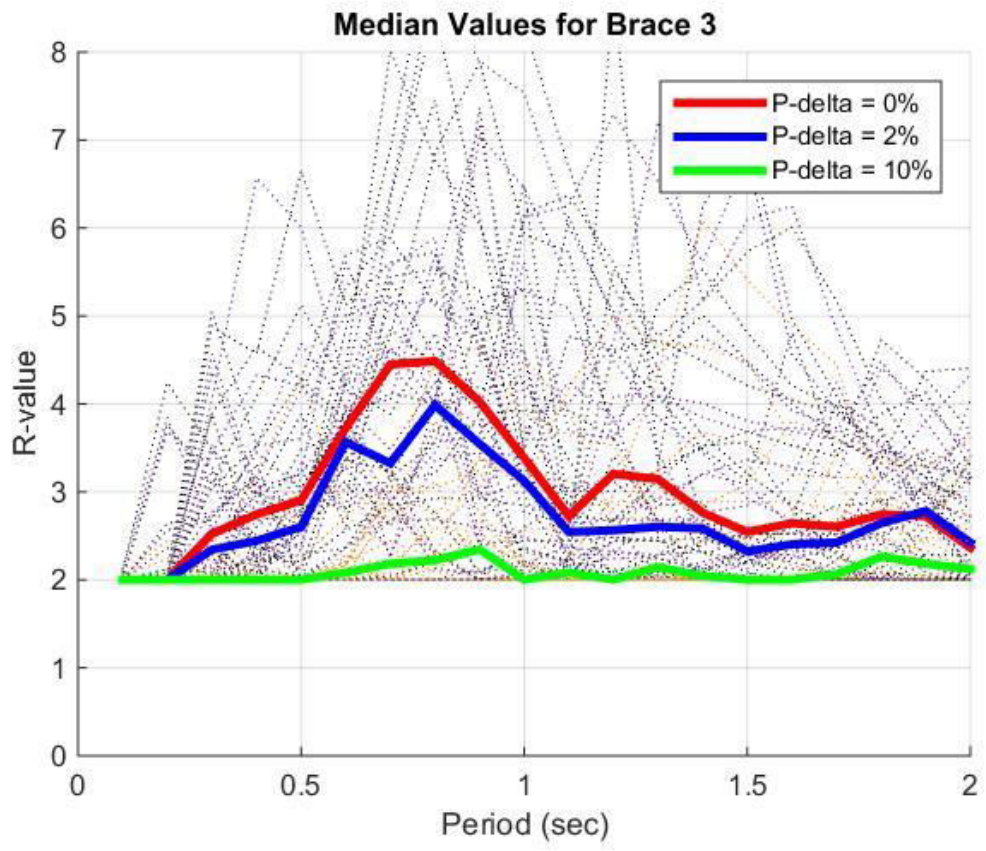

Figure 5.3.c: Median leaning column effects on Brace 3 under the EQ suite.

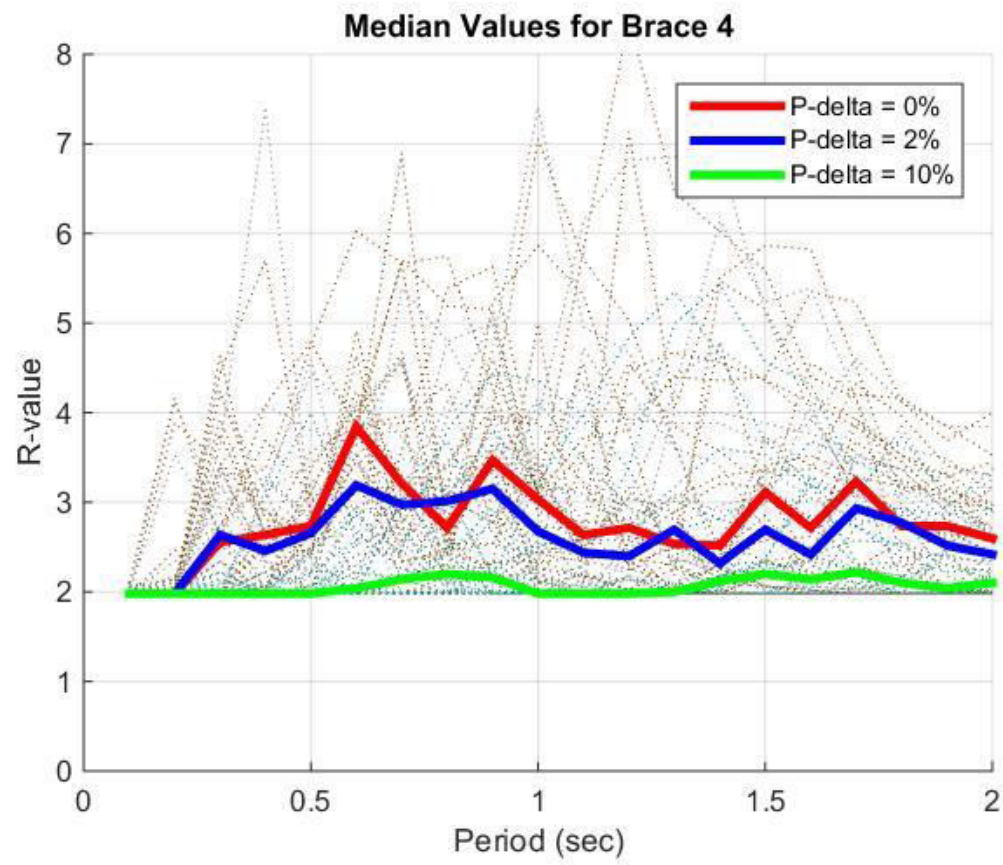

Figure 5.3.d: Median leaning column effects on Brace 4 under the EQ suite. 
Based on the data for the entire earthquake suite the following trends are observed: (1) the R-factor is reduced with an increase in the lean column effect, and (2) maximum values for $\mathrm{R}$-factor are in the 0.5 second to 1.0 second range and Brace 2 contains the largest R-factor peaks. These trends support the propposed reasonings given earlier in the section. Analyzing the median values multiple earthquakes impproved correlation of the data by reducing variance unique to specific earthquake properties. Median values are used over mean values to reduce the effects of outliers, thereby given a general response of the system. However, outliers still need to be considered in order to determine why the system isn't behaving as expected. There is to much variance in this representation of the data to come to any conclusions about the effects of slenderness on the braces' ability to support leaning columns, and the effects of the frequency of the system. It is imortant to note the the median value in this set of figures may not be fully representative since R-factors in Brace 2, 3, and 4 have a minimum of value of 2 instead of 1 , this is particularly true for P-delta $=10 \%$.

The final means of data representation in this section is the normalized reduction in the R-factor due to P-delta effects for the entire earthquake suite and the median values per brace. Shown in Figure 5.4.a to 5.4.d below. 


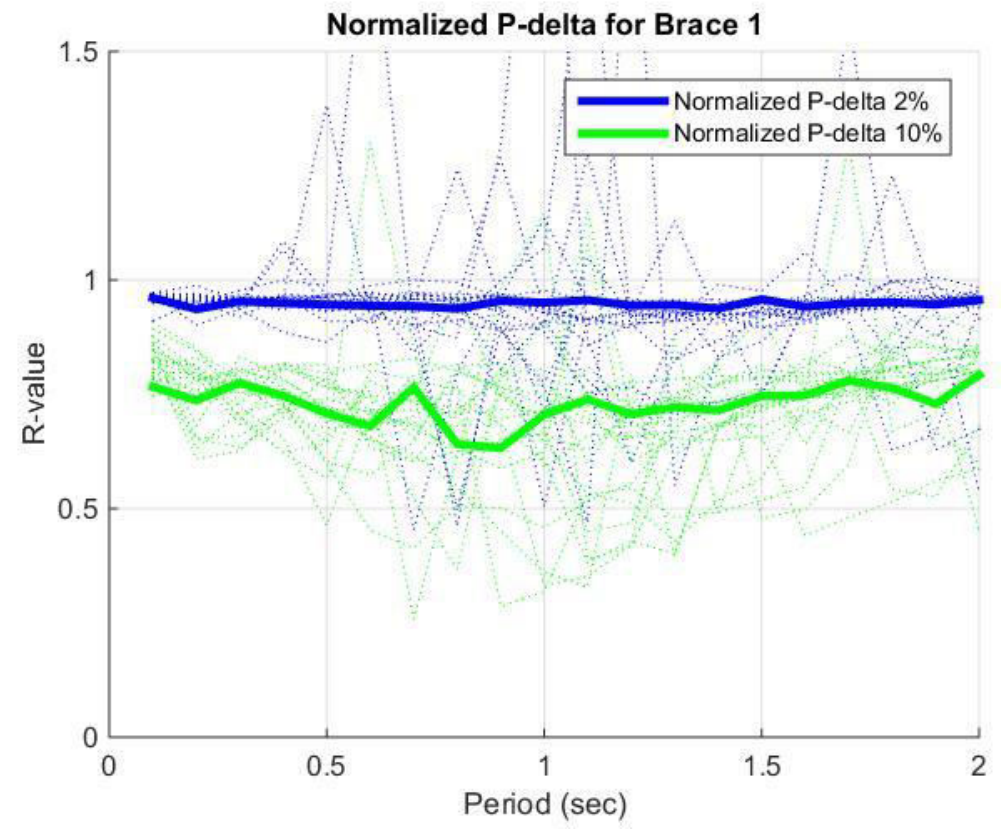

Figure 5.4.a: Normalized leaning column effects on Brace 1 under the EQ suite.

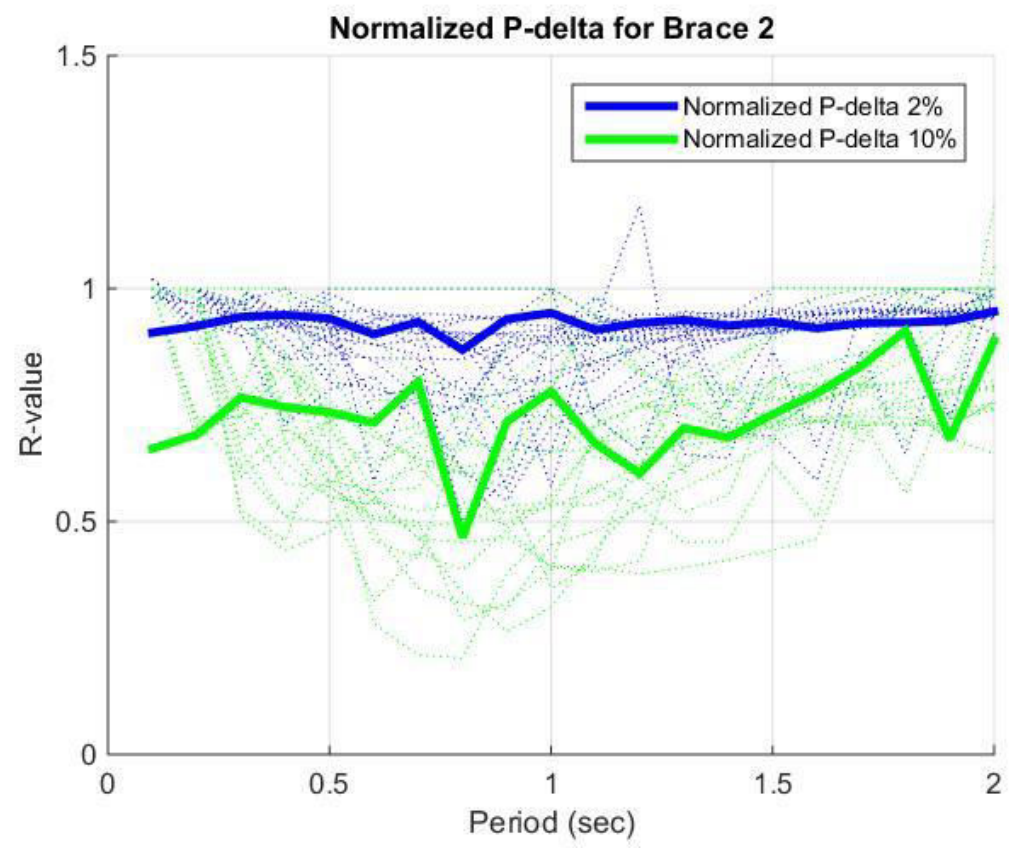

Figure 5.4.b: Normalized leaning column effects on Brace 2 under the EQ suite. 


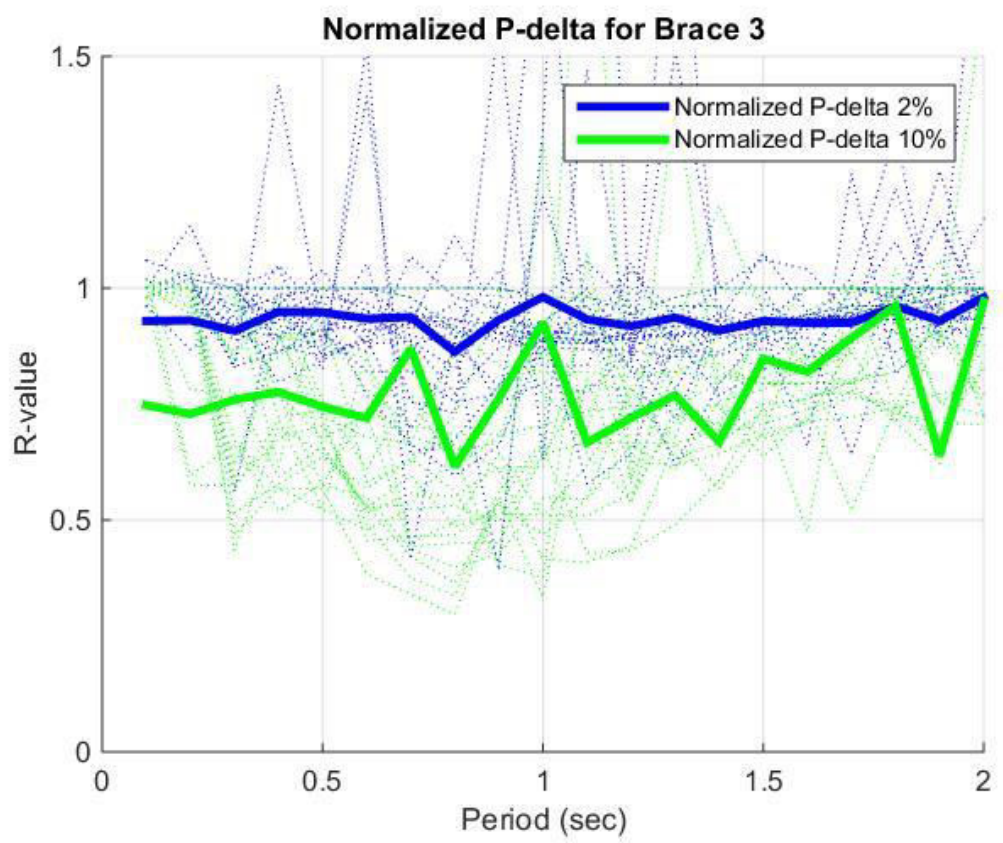

Figure 5.4.c: Normalized leaning column effects on Brace 3 under the EQ suite.

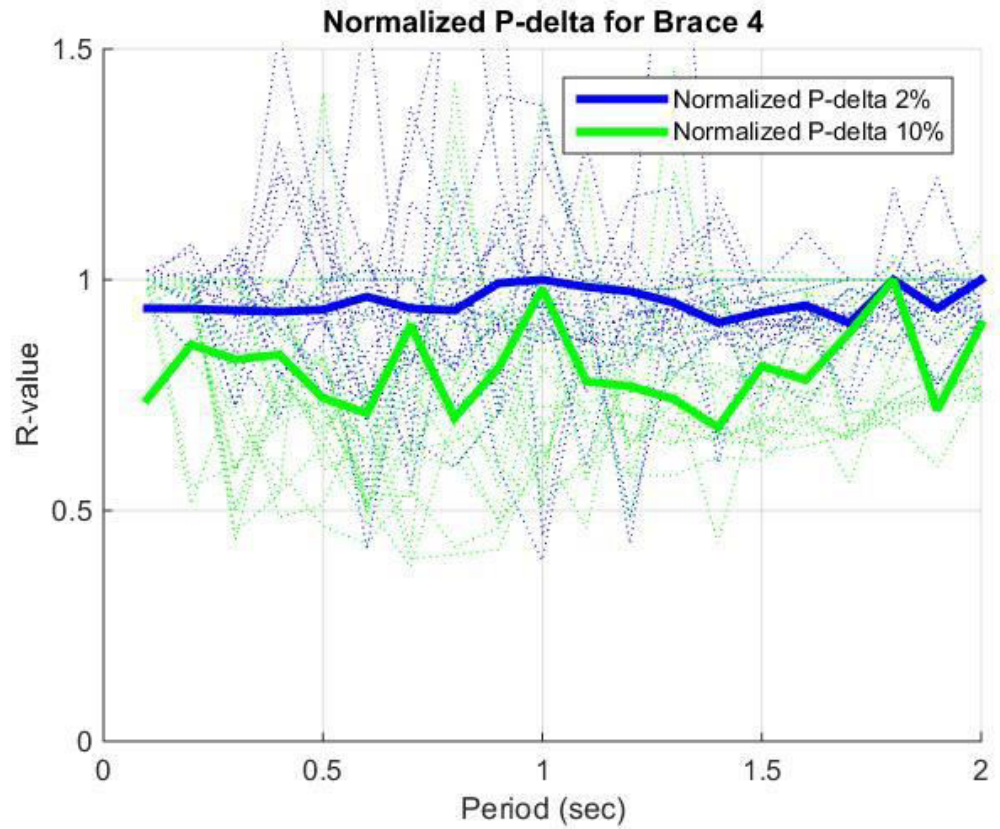

Figure 5.4.d: Normalized leaning column effects on Brace 4 under the EQ suite. 
Based on the normalized data for the entire earthquake suite the following trends are observed: (1) the R-factor is reduced at a relatively consistent and proportionate for Brace 1, and (2) the other braces behave in a more eratic manner and is therefore difficult to state any trends in this data representation with confidence. As stated earlier the data may not be fully representative of the actual values since the minimum calculated value is an R-factor of 2 . Therefore there may be a larger gap (e.g. lower normalized R-factor values) in the data, particularly for P-delta $=10 \%$. Updated values may improve the consistency and proportionality of the normalized data.

\subsection{Fatigue}

As stated previously, the purpose of this thesis to capture the effects that slenderness has on low-cycle fatigue. Since many other papers have analyzed the effects of lowcycle fatigue on hysteretic response [Huang and Mahin 2010; Li and Fahnestock 2013; Uriz and Mahin 2008; Krawinkler 2009; Sanchez-Zamora 2013], cyclic analysis is not considered. Little to no information would be gained from pushover analysis about fatigue and is therefore also not considered. In this thesis fatigue is considered using an IDA study. Discussion on IDA and its specifics can be found in Chapter 3. Discussion of the material used to capture the cumulating effect of fatigue can be found in Chapter 4 . All other parameters are set to default values recommended/chosen in preliminary studies.

\subsubsection{Incremental Dynamic Analysis}

The IDA study was done for all 20 earthquakes with fatigue considered and with fatigue neglected. All comparisons of performance in this section are though the R-factor of the system. The data in this section is presented in several means in order to attempt to gain insight of how fatigue effects the braces. The first representation of data is shown below 
in Figure 5.5.a to 5.5.d. This representation shows how the system is effected when fatigue is considered during a single considered earthquake.

Unfortunately, this representation of the data does not give any viable insight on the effects of fatigue as there is scatter in the data. The fatigue material doesn't add any stiffness to the system, so the system should result in lower R-factor values with fatigue on. The fatigue material has a more strict strain routine that results in an accurate solution. The more accurate solution could result in a different solution, potentially larger, than when fatigue is not considered (e.g. Resurrection). Finer mesh refinement should solve this issue but will increase computational efforts.

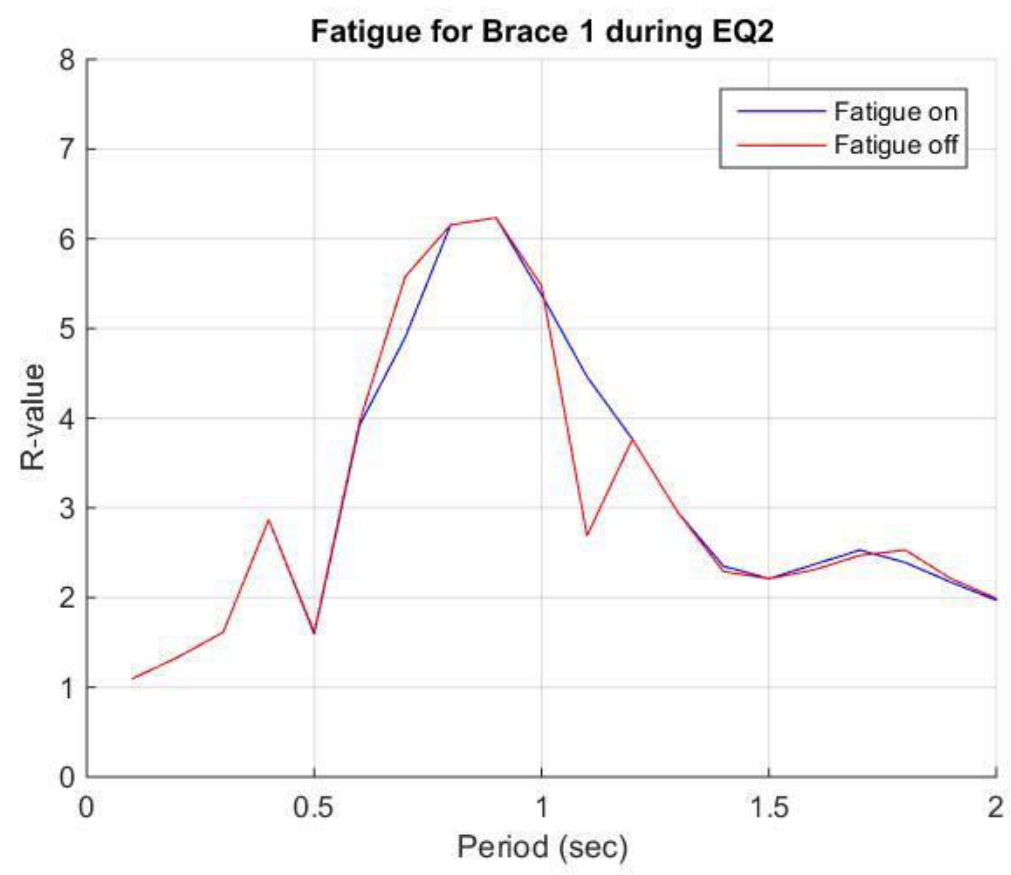

Figure 5.5.a: Fatigue consideration for Brace 1 under 1995 Kobe Earthquake. 


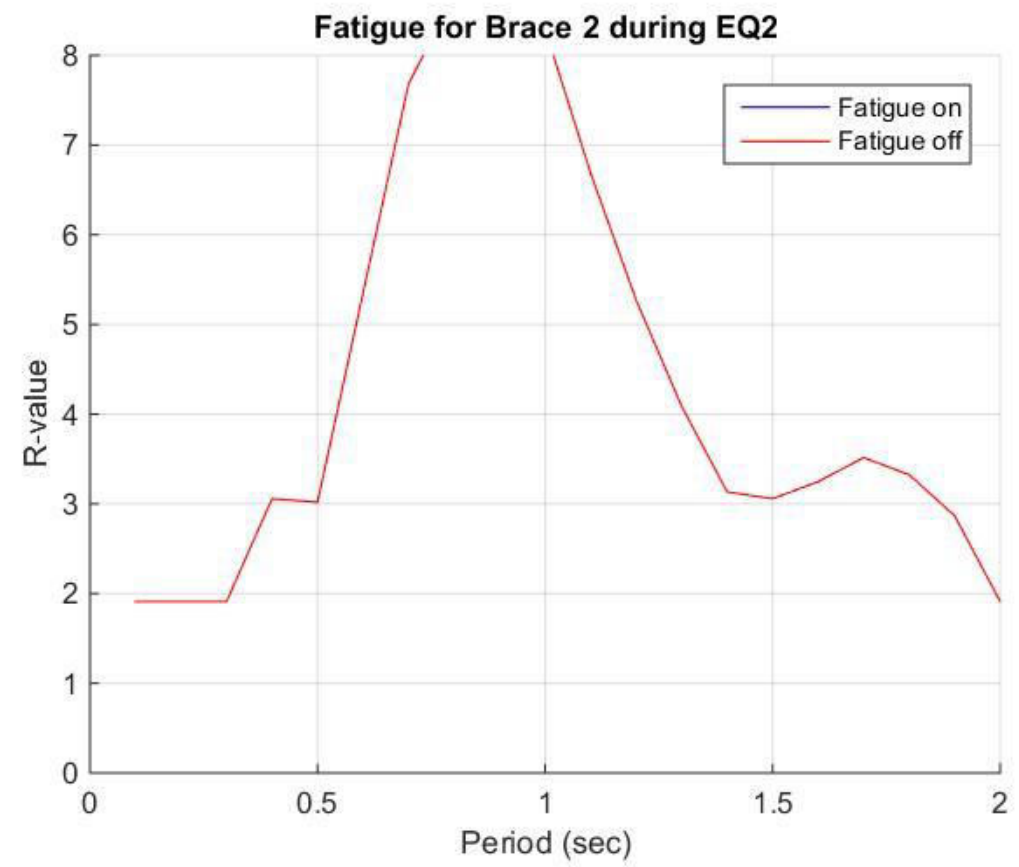

Figure 5.5.b: Fatigue consideration for Brace 2 under 1995 Kobe Earthquake.

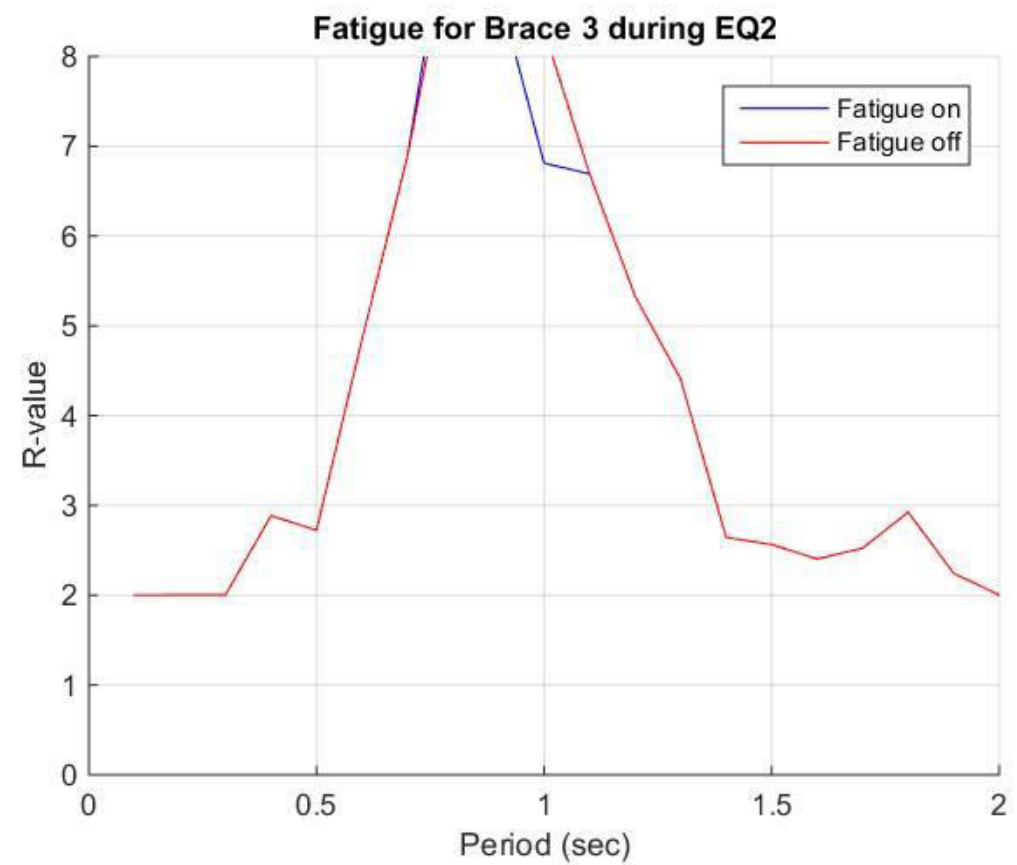

Figure 5.5.c: Fatigue consideration for Brace 3 under 1995 Kobe Earthquake. 


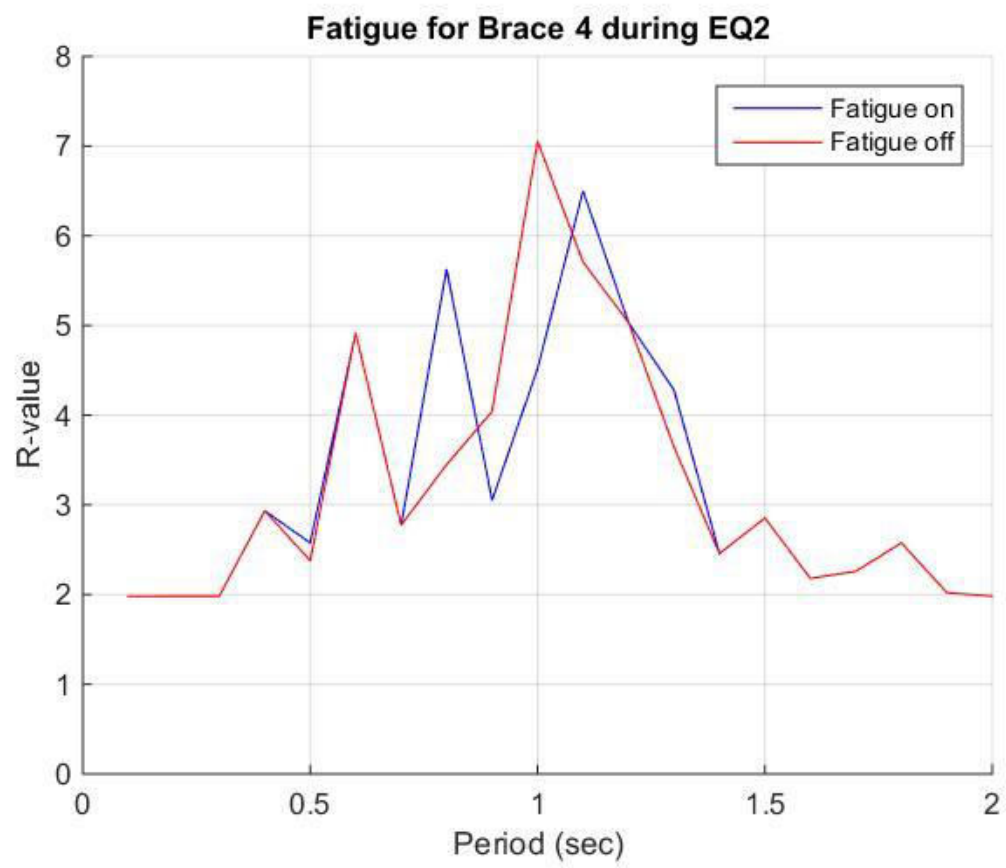

Figure 5.5.d: Fatigue consideration for Brace 4 under 1995 Kobe Earthquake.

Since little information was gained from analyzing a single earthquake, besides the possible occurrence of Resurrection, the next representation of data considers all earthquakes and their median value. 


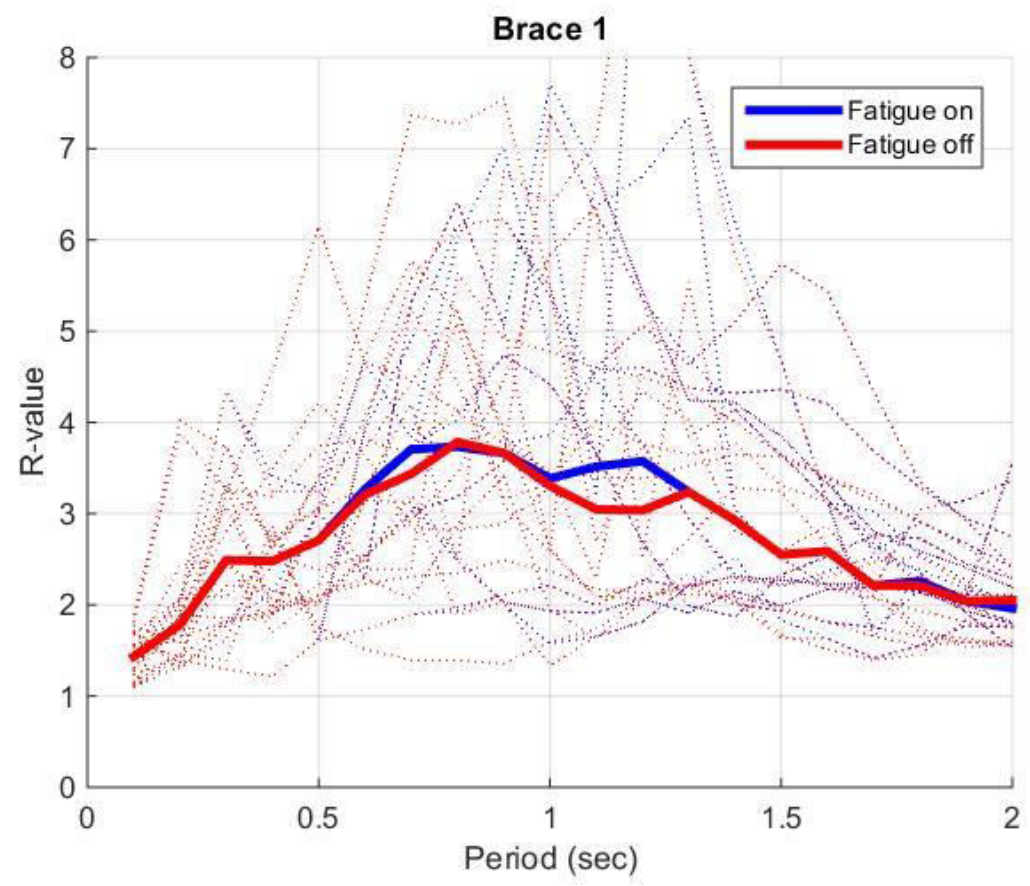

Figure 5.6.a: Fatigue consideration for Brace 1 under the EQ suite.

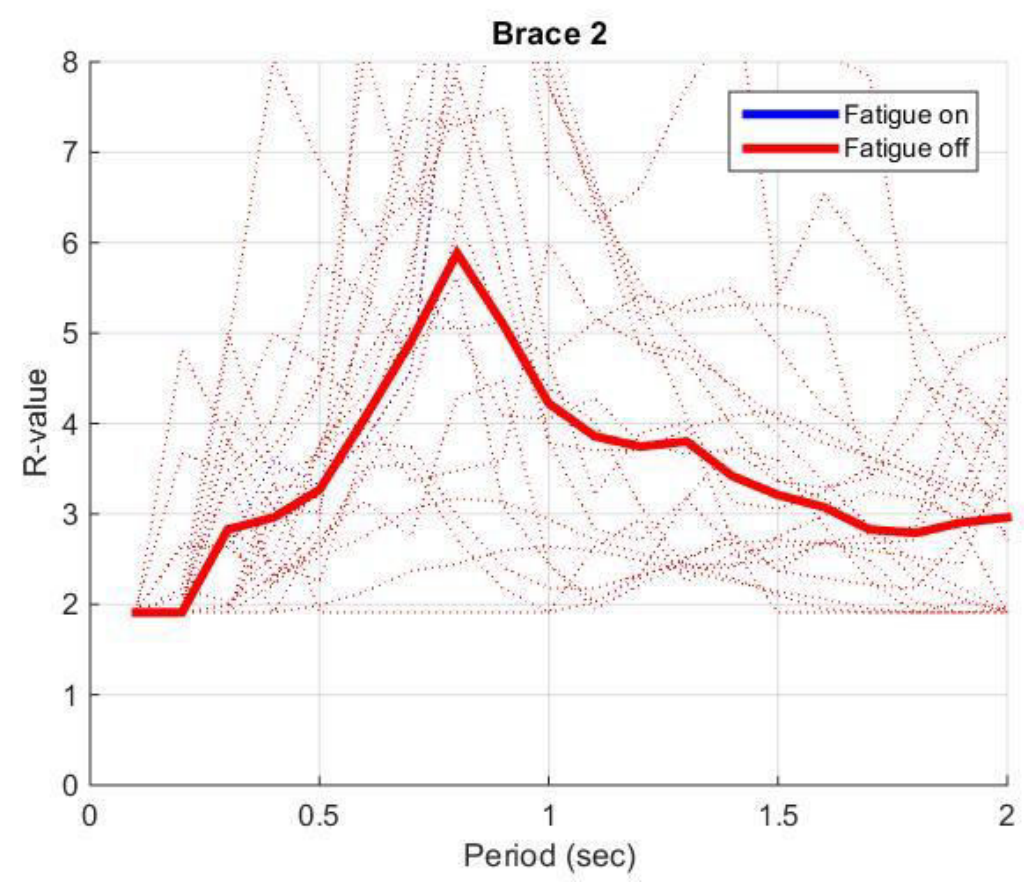

Figure 5.6.b: Fatigue consideration for Brace 2 under the EQ suite. 


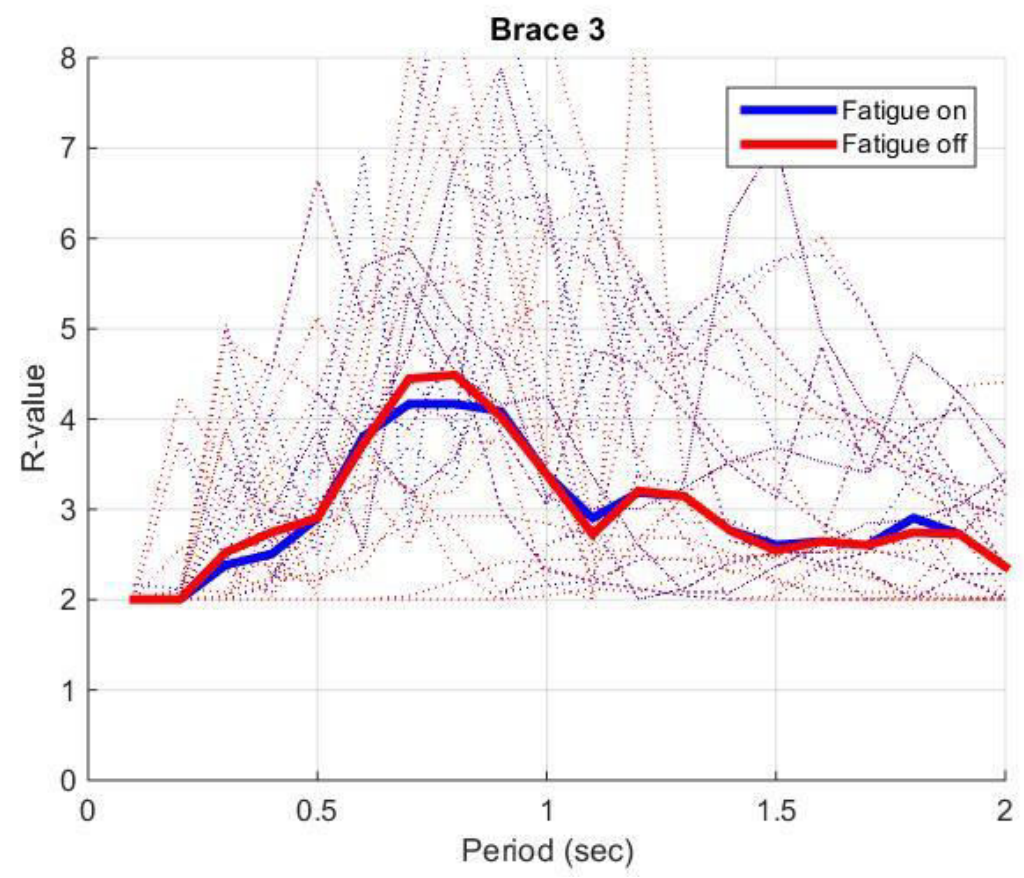

Figure 5.6.c: Fatigue consideration for Brace 3 under the EQ suite.

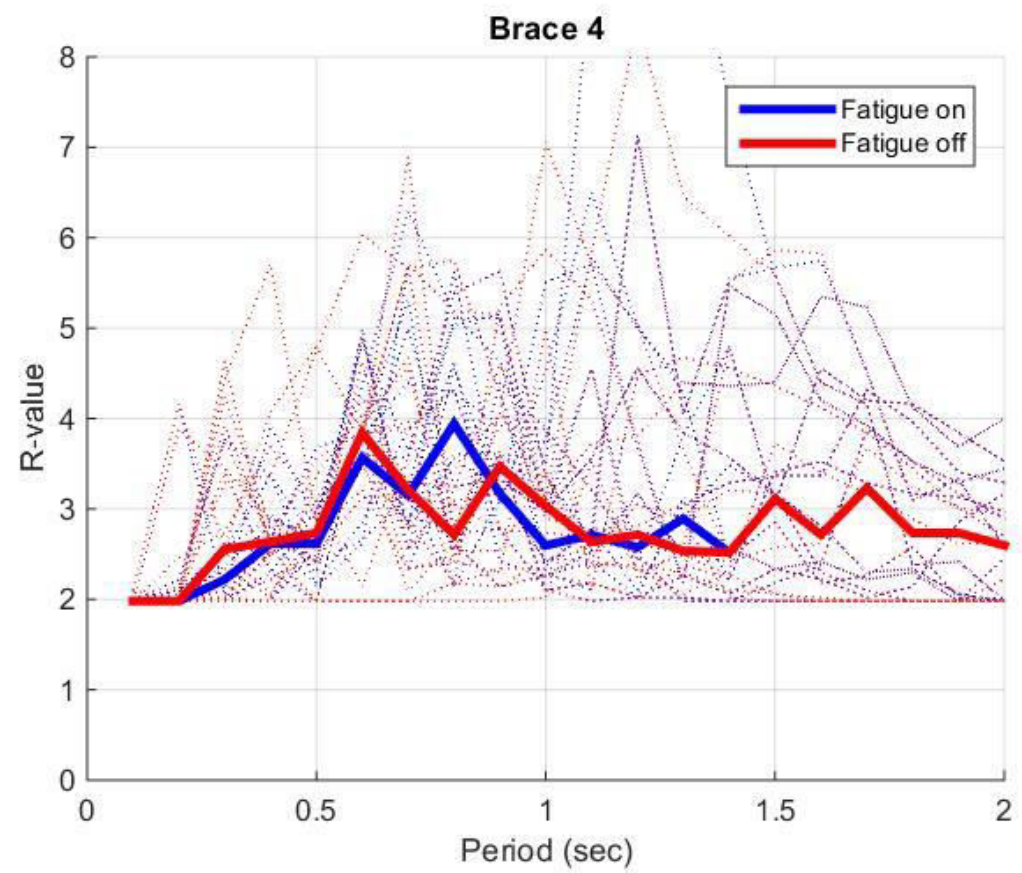

Figure 5.6.d: Fatigue consideration for Brace 4 under the EQ suite. 
When looking at the median of the earthquake suite it is still difficult to correlate any trends regarding slenderness with confidence due to the scatter above and below the control (e.g. fatigue off). Mean values result in a similar scatter to median values.

However, it can be stated with confidence that fatigue does have a substantial effect on the system. Brace 2 is an exception to this effect and will be discussed in the normalized representation of the data.

The final means of data representation for fatigue is a normalized visualization where Rfactors with fatigue considered are normalized by R-factors with fatigue not considered.

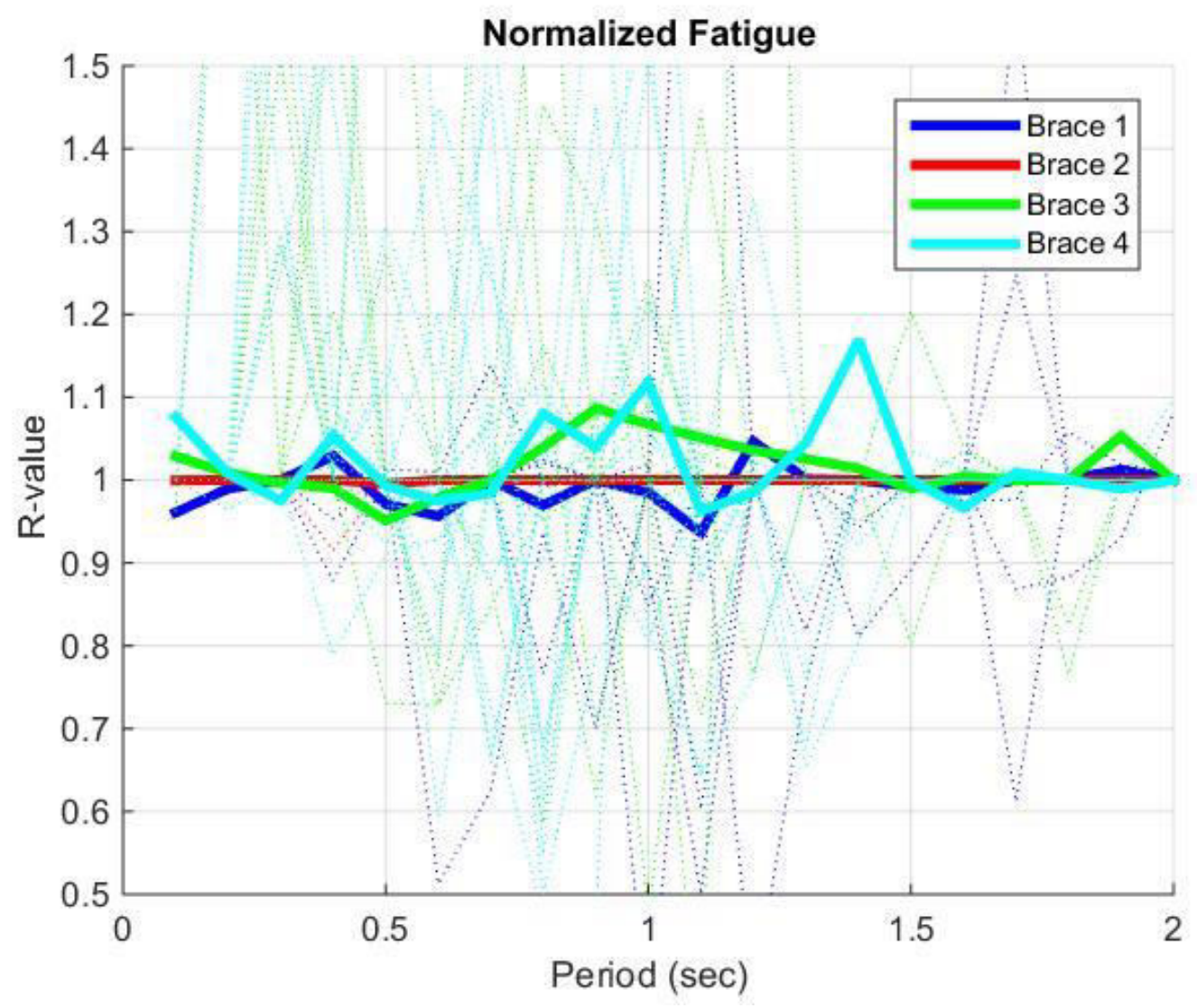

Figure 5.7: Normalized representation of the mean effects of fatigue. 
The normalized representation in Figure 5.7 above shows the relative effects of considering low-cycle fatigue in the system. Thick lines represent the mean of the ratios per each brace. Brace 4 seems to be the most sensitive to the effects of low-cycle fatigue with a maximum of $17 \%$ difference in the R-factors when fatigue is considered. Brace 3 has a maximum of $10 \%$ difference in the R-factors when fatigue is considered. Brace 1 has a maximum of $6 \%$ difference in the R-factors when fatigue is considered. And Brace 2 has negligible differences in the R-factors when fatigue is considered. The trend of the normalized data for Brace 3 and 4 being greater than one may suggest that more slender braces are more susceptible to resurrection, and therefore would require a finer mesh or a finer increment resolution. Refer to Figure 2.5 for the effects of elements sub-division with and without fatigue. Though this figure gives some insight further analysis needs to be conducted to verify if resurrection is the culprit. It is difficult to compare these results to other research due to the lack of studies on low-cycle fatigue, particularly in an IDA studies. 


\section{Conclusion}

Due to the scatter in the data for incremental dynamic analysis, any conclusion on how slenderness effects low-cycle fatigue cannot be stated with confidence. Some portions of the data show that considering low-cycle fatigue results in an increases in the $\mathrm{R}$ factor of the system. This is most likely caused by Resurrection, however, due to the complexity of this analysis there may exist several unforeseen factors effecting the results. Results of the effects of P-delta are well defined but show that slenderness has little effect of the system's ability to resist leaning columns.

The SDOF system used in this thesis is analyzed to provide a means of comparison of performance for many different types of structures. However, there are many assumptions used that could have an adverse effects on the results and must be considered when making use of the data for comparisons/correlations. First, the two dimensional model itself. The simplified model does not take into consideration factors facing a complex three dimensional system including: higher order modes, out of plane effects, soft stories, torsional effects, added stiffness from walls and diaphragms, boundary elements that aren't ideally ridged, beam-column interface detailing, member proportioning, and the effects of gusset plates. Second, the HSS braces. The braces analyzed in this thesis are not available in practice, therefore the response of real HSS braces would have to be correlated which may not fully representative. Also several assumptions are made during analysis of the braces including: neglecting of local buckling, neglecting fracture mechanics and crack propagation, and assuming plane sections remain plane. Lastly, the analytical model should be calibrated with experimental data to improve the accuracy and precision of the results under complex loading. 


\subsection{Improvements in Current Model}

Addressing any of the limitations/assumptions listed on the previous page will improve the model. However, the issues due to Resurrection should be handled prior. To attempt to reduce Resurrection the FEA mesh could be further refined and/or scaling factor steps could be reduced during IDA. It may also be beneficial to redefine the initial scaling factor definition to ensure that Brace 2, 3, and 4 start at an R-factor of 1.0, like Brace 1.

Mesh refinement can be achieved by sub-diving the braces into more elements, increasing the number of fibers in the cross section, or increasing the number of integration points. Increasing the number of elements per brace is a recommended starting point. In this model 20 sub-elements are used during IDA while considering fatigue, this is the minimum recommended value of sub-divisions when considering fatigue [Uriz and Mahin 2008]. Refer to Figure 2.5 for the effects of the brace subdivision. The number of fibers in the cross section is above the recommended minimum and therefore most likely doesn't need to be increased. If any more fibers are introduced they should be applied to the outer perimeter of the cross section. A default value of 3 Gauss integration points is used, 3 points are required to ensure the equations aren't under integrated but adding more seems to have little effect.

Use of smaller incremental steps greatly decreases computational efficiency but will improve results. If smaller incremental steps are required then it is recommended that it be implemented in a hybrid fashion. Use the current incremental step and find where failure first occurs. Starting at an initial scaling factor slightly less than the scaling factor associated with failure, rerun IDA with a finer increment. This method should reduce Resurrection while only slightly adding computational costs. 


\subsection{Recommendations for Future Research}

Since the analytical data is inconclusive for the effects that slenderness has low-cycle fatigue, it is recommended that further analysis be conducted. Analysis can be conducted in a similar manner used in this thesis with the recommended improvements or an entirely different approach could be used. The effects of low-cycle fatigue could be considered using an energy approach and hysteretic curves under seismic loading. Another possible research topic could be analyzing the effects of damping on low-cycle fatigue. Once analytical methods produce reliable trends regarding low-cycle fatigue, experimental tests should be conducted to confirm response and to further calibrate the analytical model. 


\section{BIBLIOGRAPHY}

AISC. (2005). "Seismic Provisions for Structural Steel Buildings." American Institute of Steel Construction $\{$ AISC , Chicago, Illinois 60601.

AISC. (2011). "Steel Construction Manual $14^{\text {th }}$ Edition." American Institute of Steel Construction AISC, Chicago, Illinois 60601.

Archer, Graham. "Nonlinear Structural Behavior II." California Polytechnic State University, San Luis Obispo. Winter 2015. Course notes.

ASTM. (2003). "ASTM E 1049 - 85: Standard Practices for Cycle Counting in Fatigue Analysis." West Conshohocken, PA.

Ballio, G., and Castiglioni, C. A. (1995). "A Unified Approach for the Design of Steel Structures under Low and/or High Cycle Fatigue." Journal of Constructional Steel Research, 34, 75-101.

Bertero, V. V., and Popov, E. P. (1965). "Effect of Large Alternating Strains of Steel Beams." Journal of the Structural Division: Proceedings of the American Society of Civil Engineers, 91(ST1), 1-12.

Denavit, Mark D. and Hajjar, Jerome F., "Description of geometric nonlinearity for beamcolumn analysis in OpenSees" (2013). Department of Civil and Environmental Engineering Reports. Report No. NEU-CEE-2013-02. Department of Civil and Environmental Engineering, Northeastern University, Boston, Massachusetts. http://hdl.handle.net/2047/d20003280

Dong, Lily; Ian Mathew; Gregory P Rodgers (2010), "PEN 2.4," https://nees.org/resources/pen.

Ferch, R. (2004). Berkeley, CA, Personal Communication.

Filippou, F. C., and Fenves, G. L. (2004). "Earthquake Engineering: From Engineering Seismology to Performance-Based Engineering." Chapter 6: Methods of Analysis for Earthquake-Resistant Structures, Y. Bozorgnia and V. V. Bertero, eds., CRC Press.

Fisher, J. W., Kulak, G. L., and Smith, I. F. C. (1997). "A Fatigue Primer for Structural Engineers." 97-11, Advanced Technologies for Large Structural Systems (ATLSS), Lehigh University; Bethlehem, Pennsylvania, USA.

Glinka, G., and Kam, J. C. P. (1987). "Rainflow counting algorithm for very long stress histories." International Journal of Fatigue, 9(3), 223-228.

Hou, H. and Qu, B. (2016). "Influence of Cumulative Damage on Response Modification Factors of Elastic Perfectly Plastic Oscillators." Advances in Structural Engineering, DOI: $10.1177 / 1369433216630119$.

Huang and S. Mahin, "Simulating the Inelastic Seismic Behavior of Steel Braced Frames Including the Effects of Low-Cycle Fatigue," Pacific Earthquake Eng. Research Center, Berkeley, 2010, Report No. 104.

Ikeda, K., and Mahin, S. A. (1986). "Cyclic response of steel braces." Journal of Structural Engineering, ASCE, 112(2), 342-361.

Jain, A. K., and Goel, S. (1978). "Hysteresis Models For Steel Members Subjected to Cyclic Buckling or Cyclic End Moments and Buckling-Users Guide for DRAIN2D:EL9 AND EL10." UMEE 78R6, University of Michigan, College of Engineering, Ann Arbor, MI 48109-2125. 
Kisseberth, Nicholas (2015), "Workspace," https://nees.org/resources/workspace.

Krawinkler, H. (2009). "Loading Histories for Cyclic Tests in Support of Performance Assessment of Structural Components." 3rd International Conference on Advances in Experimental Structural Engineering.

Krawinkler, H., Zohrei, M., Lashkari-Irvani, B., Cofie, N., and Hadidi-Tamjed, H. (1983). "Recommendations for experimental studies on the seismic behavior of steel components and materials." Report No. 061, John A. Blume Earthquake Engineering Center, Stanford University, Stanford, California.

Lee, S., and Goel, S. C. (1987). "Seismic Behavior of Hollow and Concrete Filled Square Tubular Bracing Members." UMCE87-11, University of Michigan, College of Engineering, Ann Arbor, Ml 48109-2125.

Li, G. and Fahnestock, L. (2013). "Seismic Response of Single-Degree-of-Freedom Systems Representing Low-Ductility Steel Concentrically Braced Frames with Reserve Capacity." J. Struct. Eng., 10.1061/(ASCE)ST.1943-541X.0000623, 199211.

Li, G., Fahnestock, L., and Li, H. (2013). "Simulation of Steel Brace Hysteretic Response Using the Force Analogy Method." J. Struct. Eng., 10.1061/(ASCE)ST.1943541X.0000664, 526-536.

McKenna, F., Fenves, G. L. (2004). OpenSees command language manual, version 1.5.2, Pacific Earthquake Engineering Research Center, (http://opensees.berkeley.edu) (May 2004).

McKenna, Frank; Christopher McGann; Pedro Arduino; Joseph Allen Harmon (2010), "OpenSees Laboratory," https://nees.org/resources/openseeslab.

McKenna, Frank. OpenSees Workshop. Berkeley: UC Berkeley, 2014. PDF. http://lese.fe.up.pt/OSDPt2014/New/Apresent/D1_001_005_McKenna_OSDPT2014. pdf

McKenna, F., and Fenves, G. (2007). "Using the OpenSees interpreter on parallel computers." NEESit Report No.TN-2007-16, NEES Cyberinfrastructure Center; La Jolla, CA.

McLennan, Michael; George A. Howlett; Ann Christine Catlin (2010), "SynchroNEES: File Sharing," https://nees.org/resources/synchronees.

NEEShub. The George E. Brown, Jr. Network for Earthquake Engineering Simulation, 2009. Web.

Neuenhofer, A. and Filippou, F. (1997). "Evaluation of Nonlinear Frame Finite-Element Models." J. Struct. Eng., 10.1061/(ASCE)0733-9445(1997)123:7(958), 958-966.

Neuenhofer, A. and Filippou, F. (1998). "Geometrically Nonlinear Flexibility-Based Frame Finite Element." J. Struct. Eng., 10.1061/(ASCE)0733-9445(1998)124:6(704), 704-711.

(Oct 29, 2010). "P-delta and corotational transformation". Message posted to http://opensees. berkeley.edu/community/viewtopic.php?p=38258

OpenSees Subversion Repositories (Rev 6204) [Source code]. Available at http://opensees.berkeley.eduWebSVN/listing.php?repname=OpenSees\&path=\%2Ft runk\%2FSRC 
Popov, E. P., and Pinkey, R. B. (1969). "Cyclic Yield Reversal in Steel Building Connections." Journal of the Structural Division: Proceedings of the American Society of Civil Engineers, 95(ST 3), 327-353.

Qu, Bing. "Design of Seismic-resistant Steel Building Structures." California Polytechnic State University, San Luis Obispo. Winter 2014. Course notes.

Rodgers, Gregory P; Brian Rohler; Anup Mohan; Tanzima Islam (2011), "Batchsubmit High Volume Job Submission," https://nees.org/resources/batchsubmit.

Sabelli, Rafael, Roeder, Charles W., and Hajjar, Jerome F. (2013). "Seismic design of steel special concentrically braced frame systems: A guide for practicing engineers," NEHRP Seismic Design Technical Brief No. 8, produced by the NEHRP Consultants Joint Venture, a partnership of the Applied Technology Council and the Consortium of Universities for Research in Earthquake Engineering, for the National Institute of Standards and Technology, Gaithersburg, MD, NIST GCR 13-917-24

Sanchez-Zamora, F. Seismic rehabilitation of steel concentrically braced frames vulnerable to soft-story failures through implementation of rocking cores. M.S. Thesis, California Polytechnic State University, San Luis Obispo, California, 2013.

Shaback, B., and Brown, T. (2003). "Behavior of square hollow structural steel braces with end connections under reversed cyclic axial loading." Canadian Journal of Civil Engineering, 30, 745-753.

Spacone, E., Filippou, F. C., and Taucer, F. F. (1996). "Fiber Beam-Column Model for Nonlinear Analysis of RC Frames: I: Formulation." Earthquake Engineering and Structural Dynamics, 25(7), pp. 711-725.

Stojadinović, B. (2003). "Stability and Low-Cycle Fatigue Limits of Moment Connection Rotation Capacity." Engineering Structures, 25(5), 691-700.

Structural Engineers Association of California (SEAOC), Applied Technology Council (ATC), Consortium of Universities for Research in Earthquake Engineering (CUREE) (2011). "SAC Steel Project Database," Network for Earthquake Engineering Simulation (database), Dataset, DOI:10.4231/D3FQ9Q536.

"TOP500 Supercomputing Sites." top500.org, 1993-2015. Web.

Towns, John, Timothy Cockerill, Maytal Dahan, Ian Foster, Kelly Gaither, Andrew Grimshaw, Victor Hazlewood, Scott Lathrop, Dave Lifka, Gregory D. Peterson, Ralph Roskies, J. Ray Scott, Nancy Wilkins-Diehr, "XSEDE: Accelerating Scientific Discovery", Computing in Science \& Engineering, vol.16, no. 5, pp. 62-74, Sept.-Oct. 2014, doi:10.1109/MCSE.2014.80

Tremblay, R., Archambault, M.-H., and A., F. (2003). "Seismic Response of Concentrically Braced Steel Frames Made with Rectangular Hollow Bracing Members." American Society of Civil Engineers: Journal of Structural Engineering, 129(12), 1626-1636.

Uriz, P., Mahin S. (2008). "Toward Earthquake-Resistant Design of Concentrically Braced Steel-Frame Structures." Pacific Earthquake Engineering Center, University of California, Berkeley, 2008

Vamvatsikos, D. and Cornell, C. A. (2002), Incremental dynamic analysis. Earthquake Engng. Struct. Dyn., 31: 491-514. doi:10.1002/eqe.141 
APPENDICES

\section{A. Response Spectrum}

Figure A.1 below contains the response spectrum for the SAC earthquake suite for Los

Angeles with a probability of exceedance of $2 \%$ in 50 years. [SAC 2011]

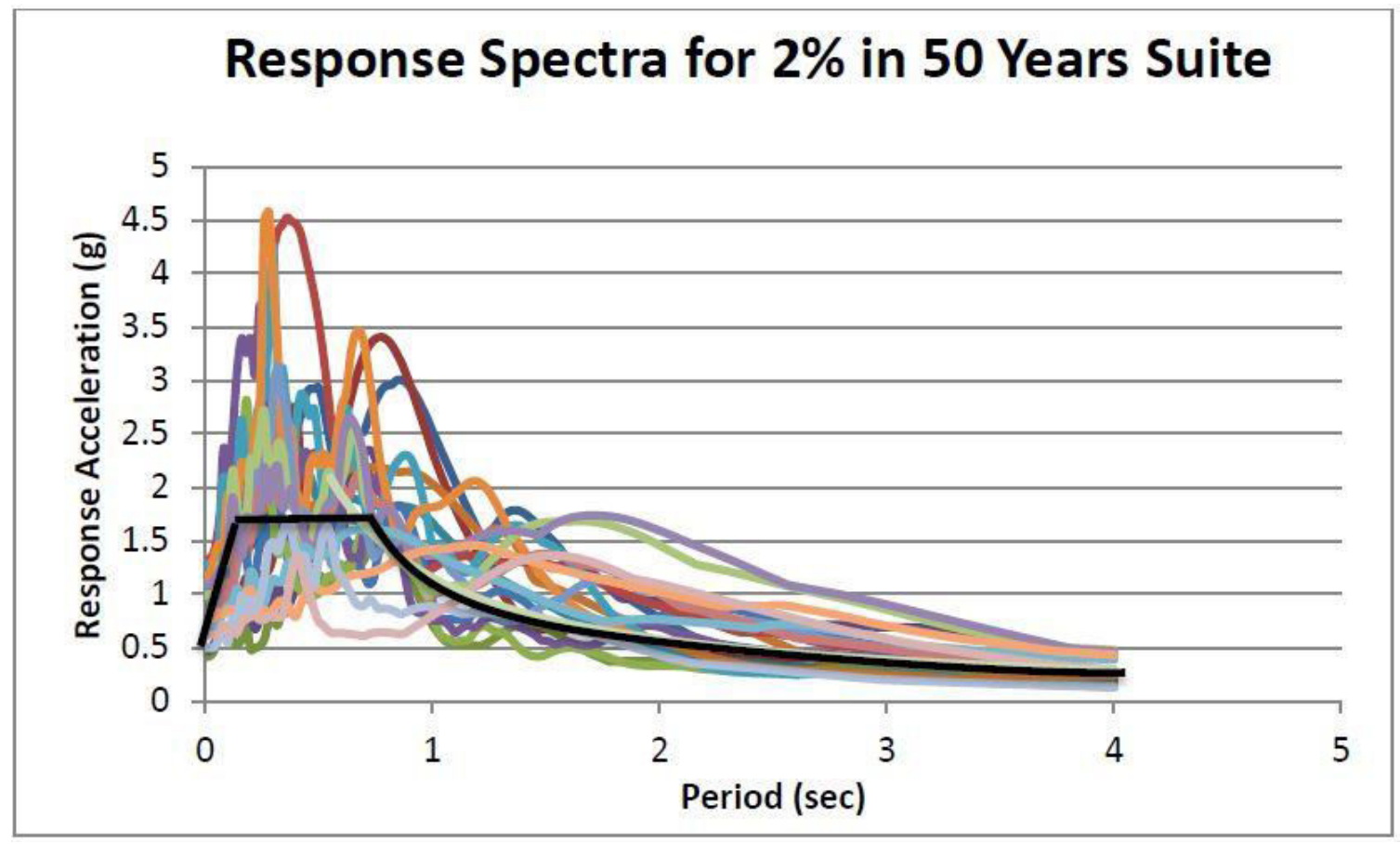

Figure A.1: MCE response spectra, image from Sanchez-Zamora 2013. 


\section{B. File Compression}

When dealing with large quantities of data, file compression helps keep data organized while reducing memory. There are many forms of compression; only .tar and .tar.gz will be discussed here. The author would like to thank Dr. Eric Kasper of Cal Poly SLO for his assistance with this compression write up.

\section{TAR}

Tar is a commonly used archiving format. The advantage of using tar is that it consumes little CPU to quickly compress files, however the compression is minimal.

To compress a directory, use the following syntax:

\# tar -cvf archive_name.tar directory_to_compress

And to extract an archive:

\# tar -xvf archive_name.tar

This will extract the files in the archive_name.tar archive in the current directory. To define the directory modify the syntax as such:

\# tar -xvf archive_name.tar -C /tmp/extract_here/

\section{TAR.GZ}

Tar.gz is similar to tar but offers improved compression while still only utilizing minimal CPU.

To compress a directory use the following syntax:

\# tar -zcvf archive_name.tar.gz directory_to_compress

To decompress an archive use the following syntax:

\# tar -zxvf archive_name.tar.gz

This will extract the files in the archive_name.tar.gz archive in the current directory. To define the directory modify the syntax as such:

\# tar -zxvf archive_name.tar.gz -C /tmp/extract_here/ 


\section{Environmental Variables And Permissions}

Ensure that the user has administrative permissions when downloading/installing

OpenSees and Tcl/Tk for the first time. The computer should automatically create an environmental path and variable for the programs. This allows OpenSees to call scripts freely. To check if the environmental path is set or to add one go to Advanced System Settings in Computer. Under the Advanced tab, click Environmental Variables button. Click on Path under System Variables and click the Edit button.

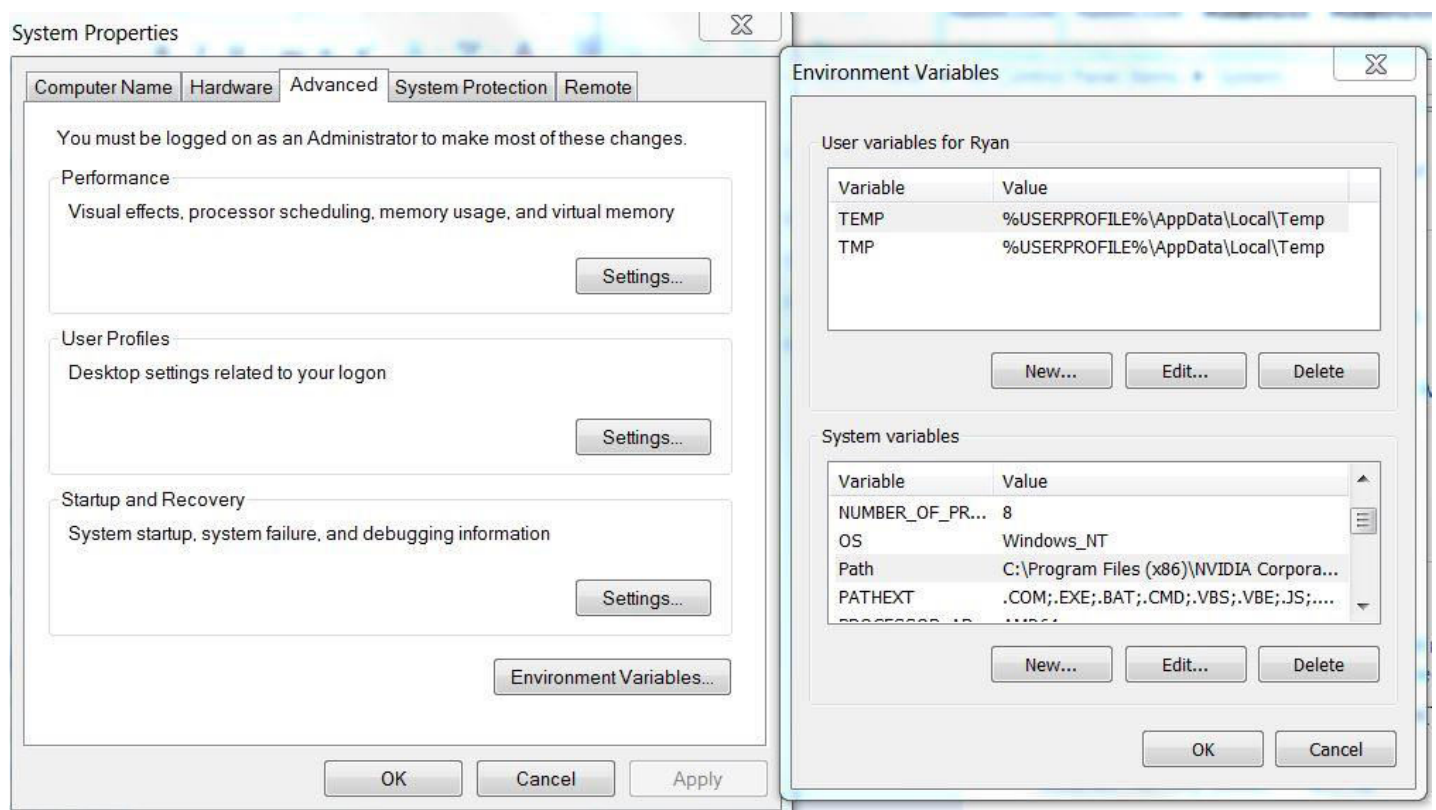

Figure C.1: Altering environmental variables.

Look for $<$ C:IProgram FilesITcllbin> in the list of Variable value, if it doesn't exist then add it to the list with a semicolon to separate paths. If there are still issues then try adding the bin for OpenSees executable using a similar format. Note users will need administrator privileges to alter system variables. If errors about read or write permissions arise then the directory is not properly setup. The easiest way to alter directory settings is through Command Prompt (cmd.exe). To change a directory in Command Prompt use the command cd. Use ICACLS or similar syntax to alter the directory. If running on third party vendors then ensure that they have the proper software installed on their servers. 


\section{Using NEEShub}

\section{Abstract (copied exactly from https://nees.org/about/overview)}

People and communities world-wide are significantly impacted when they experience an earthquake or tsunami, not only at risk of losing life or property but in dealing with the chaos caused by the disruption of services that meet basic needs of water, food and shelter.

The George E. Brown, Jr. Network for Earthquake Engineering Simulation (NEES) was created by the National Science Foundation (NSF) to aggressively move forward the development of improvements and innovations in infrastructure design and construction practices to prevent or minimize damage during such an event.

Earthquake engineering researchers and students have the opportunity through the NEES collaboratory of 14 experimental equipment sites and a robust cyberinfrastructure featuring online simulation tools to conduct more advanced research of designs, materials, construction techniques and monitoring tools. Research results will enable engineers to develop better and more cost-effective ways of mitigating earthquake damage.

To run OpenSees scripts on NEEShub follow the steps listed:

1. Create an account with a valid .edu email address

2. Submit a Support Ticket to NEEShub requesting access to High performance Computing Access, this will grant the user access to run simulations on Teragrid servers.

3. If the user is planning on collecting a lot of data with NEEShub, it is recommended that the user submits another Support Ticket. Requesting that the allotted memory be increased from $1 \mathrm{~GB}$, the default, to $10 \mathrm{~GB}$.

4. If the user is working with other researchers, a group can be created where users can share files and data.

5. To run the OpenSees script several two main methods exist. One is the use of Batchsubmit, for script savvy users this is the recommended method. The other is the use of OpenSees Laboratory, this application is a GUI and is recommended for users that prefer that kind of interface. Write ups for the use of both applications are in following sections of this appendix.

6. After the scripts have been successfully ran the user can download the data using SynchroNEES or PEN. SynchroNEES can be ran directly from the desktop and is the easier application to use when downloading individual or a small group of files. However due to SynchroNEES' instability it is recommended that PEN be used when downloading large quantities of data. 


\section{OpenSees Laboratory: Parallel Job Submission \\ Abstract (copied exactly from https://nees.org/tools/openseeslab)}

This set of simulation tools has been developed for use with the OpenSees software.

There are 3 basic tool types: 1. Tools for submitting OpenSees scripts to OpenSees interpreters running on sequential and NSF Teragrid resources. 2. Tools for Educational use to instruct students on the response of structures. 3. Useful Tools for performing practical tasks. These tools will be updated constantly. Any questions, comments, difficulties should be directed to openseessupport@berkeley.edu.

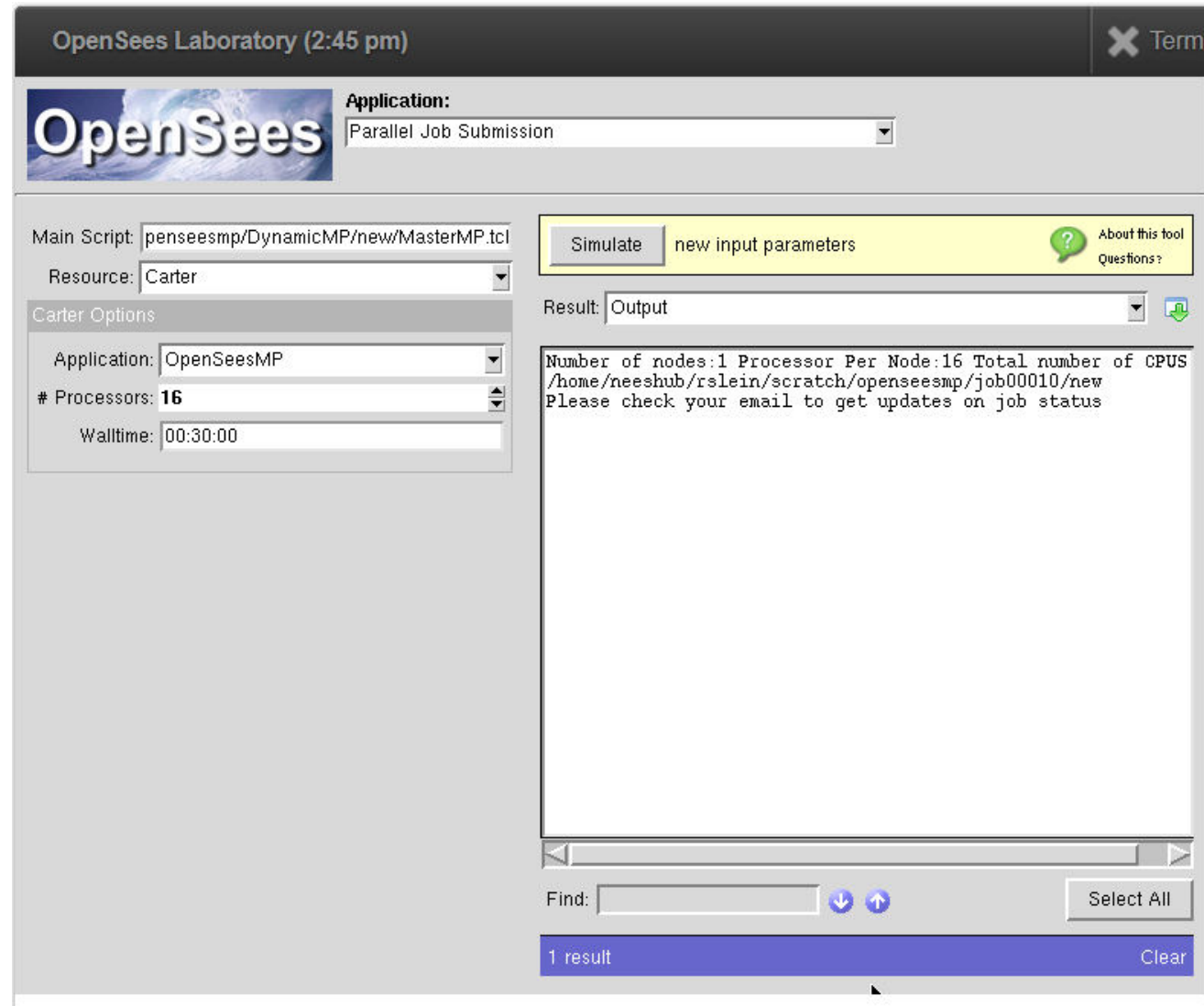

Figure D.1: OpenSees Laboratory main screen.

\section{Main Script Path}

Ensuring that the main script path is properly set is essential! Once it is set it is recommended that it is never changed, just change the files with in the folder from the path. My path is:

/home/neeshub/rslein/scratch/openseesmp/DynamicMP/new/MasterMP.tcl /home/neeshub/bqu/ryan/DynamicMP.tcl

Containing folders can be written from SynchroNEES, explained in the next section. The main script is the script that initializes the program and then calls in other scripts to build the model and to run analysis.

Ensure all files being called in are within the same folder as the main script, i.e. I need to make sure my mass.tcl is in /openseesmp/DynamicMP/new/ under my scratch space. 


\section{Program Venues}

The following venues can be used in OpenSees Laboratory to run your files:

\begin{tabular}{|c|c|c|c|c|}
\hline Venue & ncpus & $\mathrm{nn}$ & ppn & Walltime \\
\hline stampede & $\begin{array}{l}4096(\max ) \\
16(\min )\end{array}$ & $256(\max )$ & $16(\max )$ & 24:00:00(max,default) \\
\hline kraken & $\begin{array}{c}512(\max ) \\
12(\min )\end{array}$ & 42(max) & $12(\max )$ & 24:00:00(max,default) \\
\hline hansen & 48(max) & 12(max) & $4(\max )$ & $\begin{array}{l}\text { 720:00:00(max) } \\
\text { 24:00:00(default) }\end{array}$ \\
\hline osg & $\sim 60000$ & NA & NA & 24:00:00(default) \\
\hline carter & $64(\max )$ & $4(\max )$ & $16(\max )$ & $72: 00: 00$ (max,default) \\
\hline $\begin{array}{l}\text { local } \\
\text { (NEES) }\end{array}$ & $16(\max )$ & $1(\max )$ & $16(\max )$ & 24:00:00(max,default) \\
\hline
\end{tabular}

Using the table above pick which venue suites the size of the analysis that you need to run. Note: Carter is the recommended venue for new users, it is the only site that is dedicated for academic research and does not require any special permission.

\section{Application}

Make sure you are set to OpenSeesMP if you want to utilize the benefit of multiple processors. Make sure the script is written for the given application.

\section{Number Of Processors And Walltime}

If the expected running time of the job is more than the given walltime, you should increase the number of processors so that the execution time is reduced. If the given wall time is too short the program will stop prematurely. High quantity of processors are not needed if the analysis is not very complex, look for commands in OpenSeesMP to more evenly distribute work over multiple processors.

\section{Submitting Multiple Jobs}

You can submit up to 5 jobs at any given time. If this job limit is exceeded then the new job will be set up in scratch space but will not actually be submitted until a previous job is complete, you also won't get an email notification until that job has started.

It is recommend that you clear all previous jobs in OpenSees Laboratory before running the next simulation, this won't affect the status of previous jobs but for some reason there is a higher chance that the run won't crash if old jobs are cleared. 


\section{Program Crashes And Errors}

When submitting jobs the program can be properly set up but the run will still crash. The error will look like this in the .stderr file:

mpirun has exited due to process rank 0 with PID 28927 on

node carter-c015.rcac.purdue.edu exiting improperly. There are two reasons this could occur:

1. this process did not call "init" before exiting, but others in

the job did. This can cause a job to hang indefinitely while it waits

for all processes to call "init". By rule, if one process calls "init",

then ALL processes must call "init" prior to termination.

2. this process called "init", but exited without calling "finalize".

By rule, all processes that call "init" MUST call "finalize" prior to

exiting or it will be considered an "abnormal termination"

This may have caused other processes in the application to be terminated by signals sent by mpirun (as reported here).

If this error happens check your code and keep trying to run your program, changing the wall times around sometimes helped.

There are several other errors that may occur, try checking forums for answers or ask a question on NEEShub.

\section{Writing Over Files While Running}

In the case where an early part of a program is writing output files to a location that will be read later in the program, make sure that you add a barrier command when using multiple processors. There is a chance that a file that is trying to be read has not been written yet, this will result in a crash. The barrier command will tell the processors to wait until all steps before that command are completed.

\section{Notes On OpenSees Laboratory}

- An email will be sent to your account when a job is started and completed.

- Recommend using SynchroNEES to make file transferring easier.

- By default you only have 1 GB of space on your NEES account watch out for large output files that will eat up space. $10 \mathrm{~GB}$ of spaced can be allotted with permission from a support ticket.

- Ensure that the wall time used is sufficient, if the program isn't finished by the time the walltime expires the program will stop running. One negative about using the max wall time is that if the program does not tell itself to stop you may waste time.

- OpenSees Laboratory along with all other programs have limited file types that they can read, ensure that all file extensions are compatible. For example earthquake files commonly end in .at2, .g3, .acc it is recommended that all file types be converted to a .txt or a .tcl. 


\section{SynchroNEES: File Sharing}

\section{Abstract (copied exactly from https://nees.org/resources/synchronees)}

SynchroNEES is a desktop tool for sharing files within groups. If you want to share some data with your research group or other colleagues, start by creating a group on NEEShub. Then, click on the Data Sharing tab within a group to create a "drop box," which acts as a shared folder for the group. Only members of the group can read/write data within the drop box folder; data stored there is protected and backed up, although other members of your group can overwrite it.

The SynchroNEES tool makes it easy to access your drop box folder. Download and install this tool on your desktop machine. When you run the tool, it will ask you to log in with your NEEShub username and password, and it will display the list of groups that you belong to. Clicking on any one of the groups will bring up a tab showing the drop box for the group, and you can drag/drop items into and out of the drop box. See the SynchroNEES Tutorial page for more details.

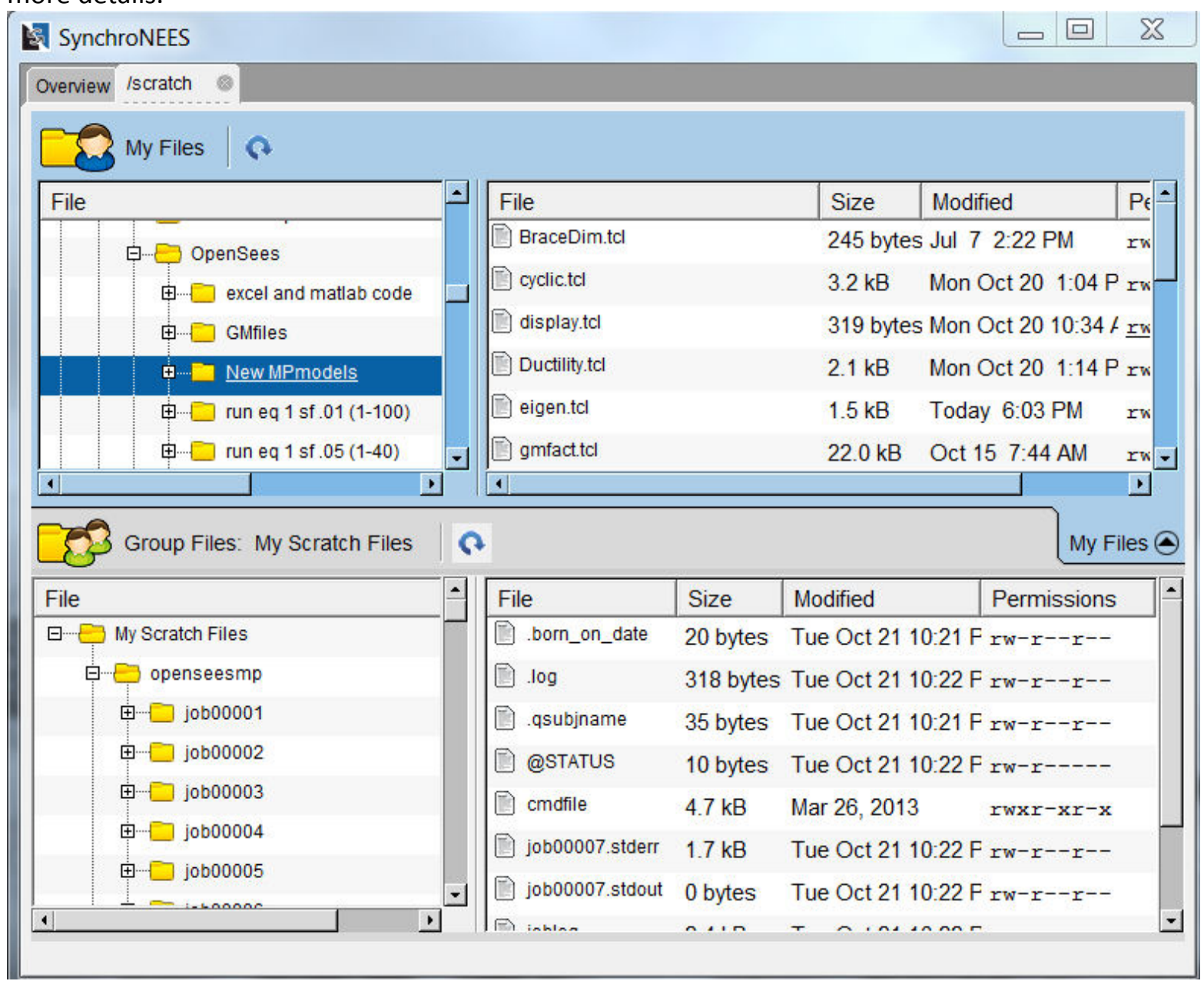

Figure D.2: Picture of SynchroNEES/scratch. 


\section{How To Add Files}

To Add Files go to My NEEShub Files. A new tab should open called /home/neeshub. Click the My Files drop down in the upper right hand corner to look at your computer files. Locate the directory to where the file you want to run are being stored.

Expand the scratch fold under the My NEEShub Files. Name the directory that you want to store you files in (very important this directory is what the master program will call upon in OpenSees Laboratory). Ensure the master is in the same folder as the files called in.

\section{How To Receive Data From Servers}

All data from completed runs can be seen under the /scratch tab which can be open from the Overview tab. A folder with the format of job00001 will be created which contains all data about the run, how it was run, and server information. job\#\#\#\#.stderr will output the window that you would see if running OpenSees on a local computer, with a few extra lines regarding the server that is running the file. This is a helpful file to troubleshoot why your job crashed!

All files used in analysis are stored in that job folder under new/lib. This is handy to check to debug any errors or if you are unsure of the parameters used in the specified job. Any files written while running the program can be found in that job folder under new/lib as well.

\section{Notes About SynchroNEES}

- You must have an NEES account registered to run this program.

- This program crashes very often! If you see the window below restart the program, none of your data will be lost.

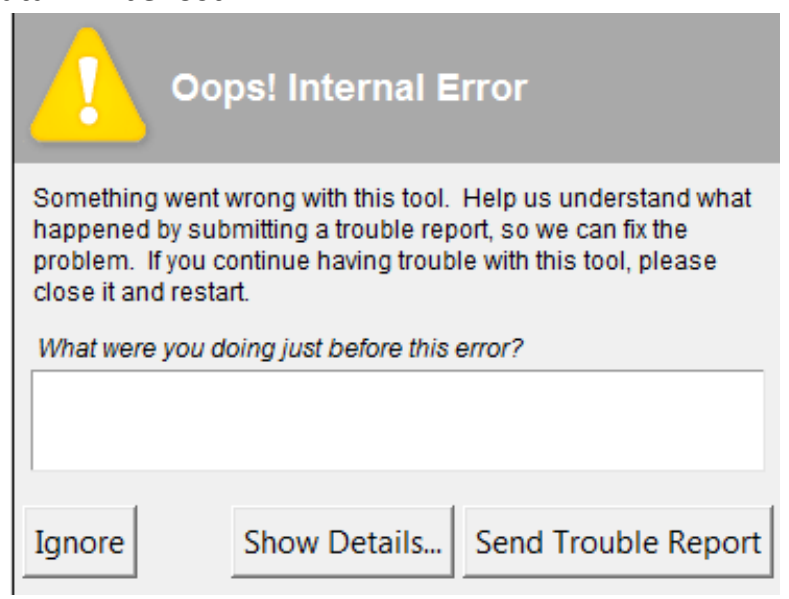

- This program has lots of bugs, always check the preview file before running test. Every file can be previewed.

- This program is the easiest way that I have found to upload data to NEES and to download finished data. NEES supports a large quantity of programs there is a chance that there may be or a more efficient program. 


\section{PEN 2.4}

\section{Abstract (copied exactly from https://nees.org/resources/pen)}

PEN is the Project Explorer for NEES. It is a java tool intended to run on a remote workstation or in the hub. The primary purpose of PEN is to organize files and directories for upload and download with the NEES Project Warehouse while a project is in active development.

\section{Notes About PEN 2.4}

Due to the frequent instability of SynchroNEES when downloading files it is recommended that PEN be used when downloading a substantial amount of files, including files in subfolders. PEN has a great tutorial that can be found at https://nees.org/wiki/PENQuickStartGuide. Due to this tutorial, little will be discussed in this section on how to run PEN; this section includes notes on issues that users may encounter.

Files can be downloaded as compressed zip files directly to your computer using the online version of PEN. Only files that exist in your project warehouse can be downloaded. To set up the directory in PEN (when using the online version through the NEEShub) go to preferences under tools. Ensure your directory is linked with your scratch space, it should look similar to the following: /home/neeshub/<rslein $>$ /scratch/

Once your directory is linked you should be find specific jobs. Drag data from the jobs you want to download into a folder in your Project Warehouse. After files can be downloaded directly as zip files. Note that PEN is unable to read many special characters including prentices, ensure that file data and titles don't contain these special characters or you will be unable to download the file.

\section{Batchsubmit}

Abstract (copied exactly from https://nees.org/tools/batchsubmit)

The Batchsubmit command provides access to a comprehensive, secure infrastructure that supports the submission, execution, and return of batch jobs. Batchsubmit was specifically created to run OpenSees batch jobs although other types of jobs can be run if the executable and all supporting files are provided.

Batch jobs can be run either locally on the NEEShub infrastructure or remotely on other platforms (venues) in serial or parallel modes. When run remotely on other venues, the Batchsubmit command transparently handles authentication and communication between NEEShub and high performance computing (HPC) venues, as well as efficient transfer of data both ways.

\section{Running Batchsubmit}

To get a full list of executable commands in Batchsubmit type the following command in the workspace:

$$
\text { batchsubmit -h }
$$




\section{E. Hand Calculations}

\section{HSS Brace Selection}

$$
\begin{aligned}
& \begin{array}{l}
\text { HSS Member Sclection } \\
\frac{K L}{r}=\frac{\sqrt{12} L_{D}}{B} \frac{1}{\sqrt{1+\left(1-2 \frac{t}{B}\right)^{2}}} \quad L_{D}=19.85^{1} \quad b / t \leq 16.07
\end{array} \\
& \text { 1) } \frac{K L}{r}=50 \quad \operatorname{Try} B=12.5 \\
& 50=\frac{\sqrt{12}\left(19.85^{\prime} \times \frac{12^{\prime \prime}}{1^{\prime}}\right)}{12.5^{\prime 1}} \frac{1}{\sqrt{1+(1-2 t / 12.5)^{2}}} \\
& t=0.862553^{n} \\
& \text { seismic esmpestness } b / t=14.49 \text { OK } \\
& \text { HSS } 12.5 \times 12.5 \times 0.86255 \\
& \text { 2) } \frac{K L}{r}=100 \text { Try } B=6.5 \\
& 100=\frac{\sqrt{12}\left(19.85^{\prime} \times \frac{12^{11}}{1}\right)}{6.5^{\circ 1}} \frac{1}{\sqrt{1+(1.2 t / 6.5)^{2}}} \\
& t=0.70849^{\prime \prime} \quad b / t=9.17 \mathrm{ok} \\
& \text { HS5 } 6.5 \times 6.5 \times 0.70849 \\
& \text { 3) } \frac{K L}{r}=200 \quad \operatorname{try} B=3.5 \\
& 200=\frac{\sqrt{12}\left(19.85^{\prime} \times \frac{12}{1}{ }^{\circ}\right)}{3.5^{\prime \prime}} \frac{1}{\sqrt{1+(1-2 t / 3.5)^{2}}} \\
& \frac{t=0.65778 \quad b / t}{H 553.5 \times 3.5 \times 0.65778}=5.3209 \mathrm{ok} \\
& \text { 4) } \frac{K L}{r}=300 \quad \operatorname{Tr} y \quad B=2.5 \\
& 300=\frac{\left.\sqrt{12}\left(19.85^{\prime \prime} \times \frac{12}{1}\right)^{\prime \prime}\right)}{2.5^{\prime \prime}:} \frac{1}{\sqrt{1+(1-2 t / 25)^{2}}} \\
& t=0.676582 \quad b / t=3.690 \mathrm{~K} \\
& \text { H5S } 2.5 \times 2.5 \times 0.67658
\end{aligned}
$$




\section{Base Shear}

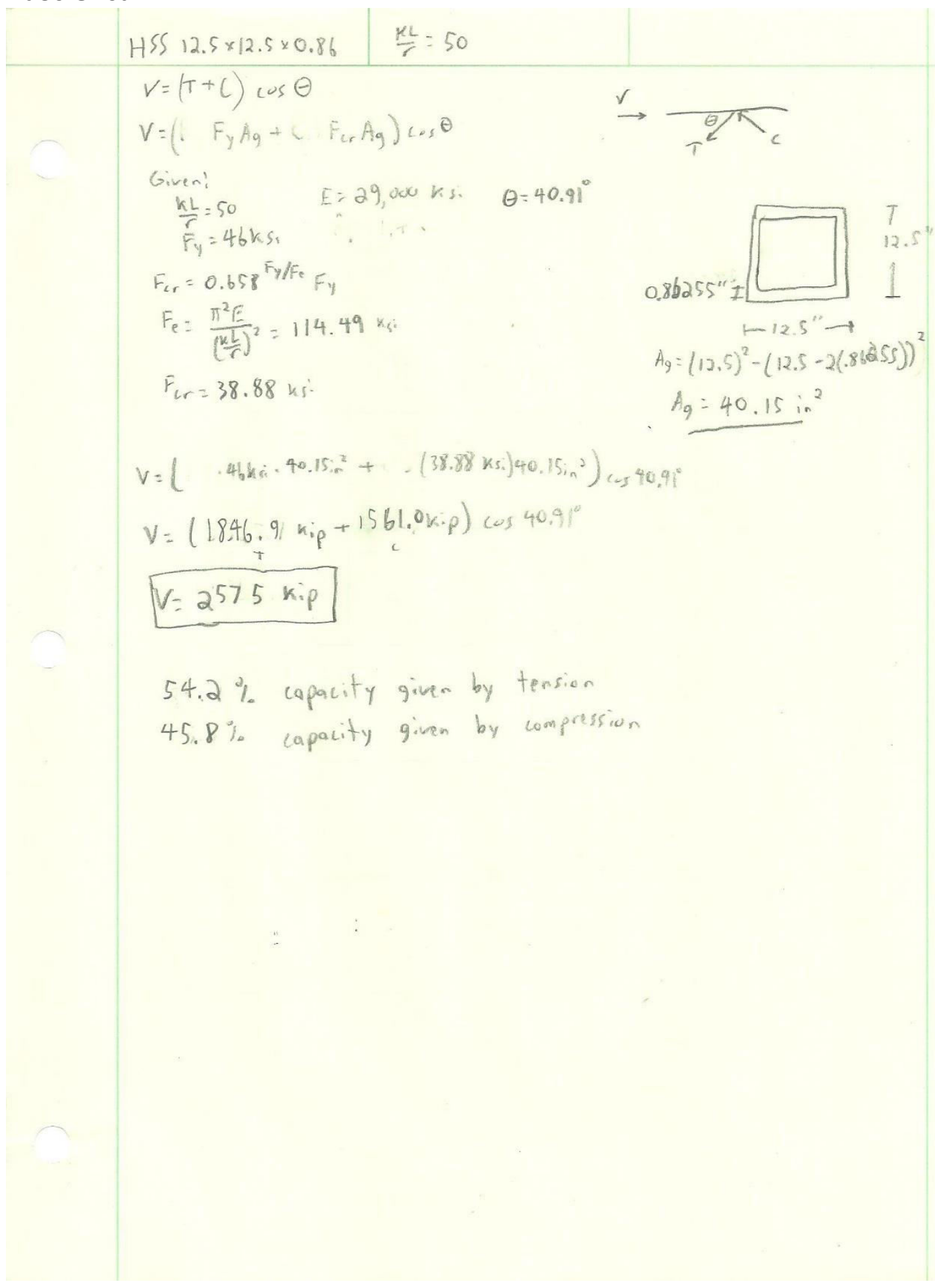




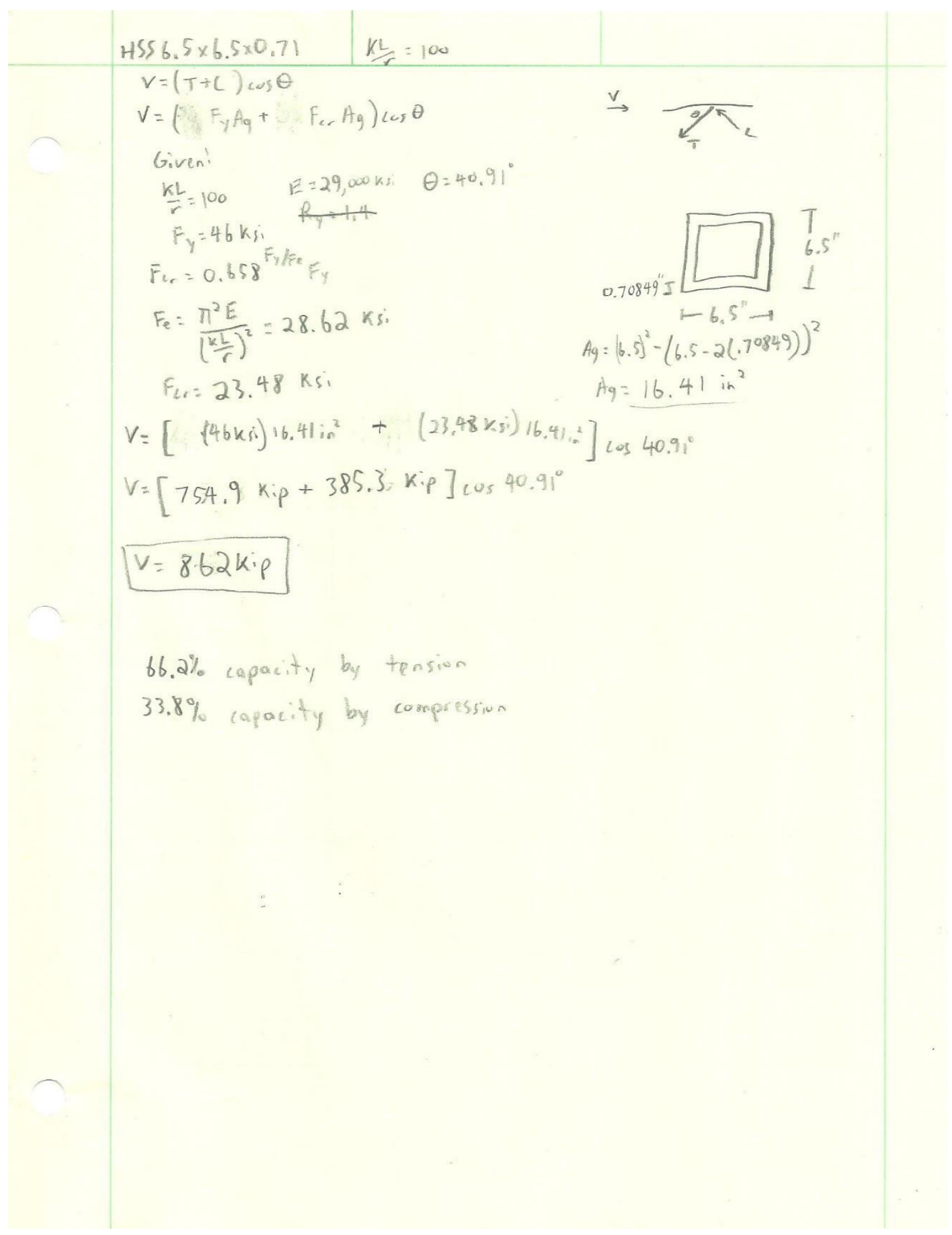




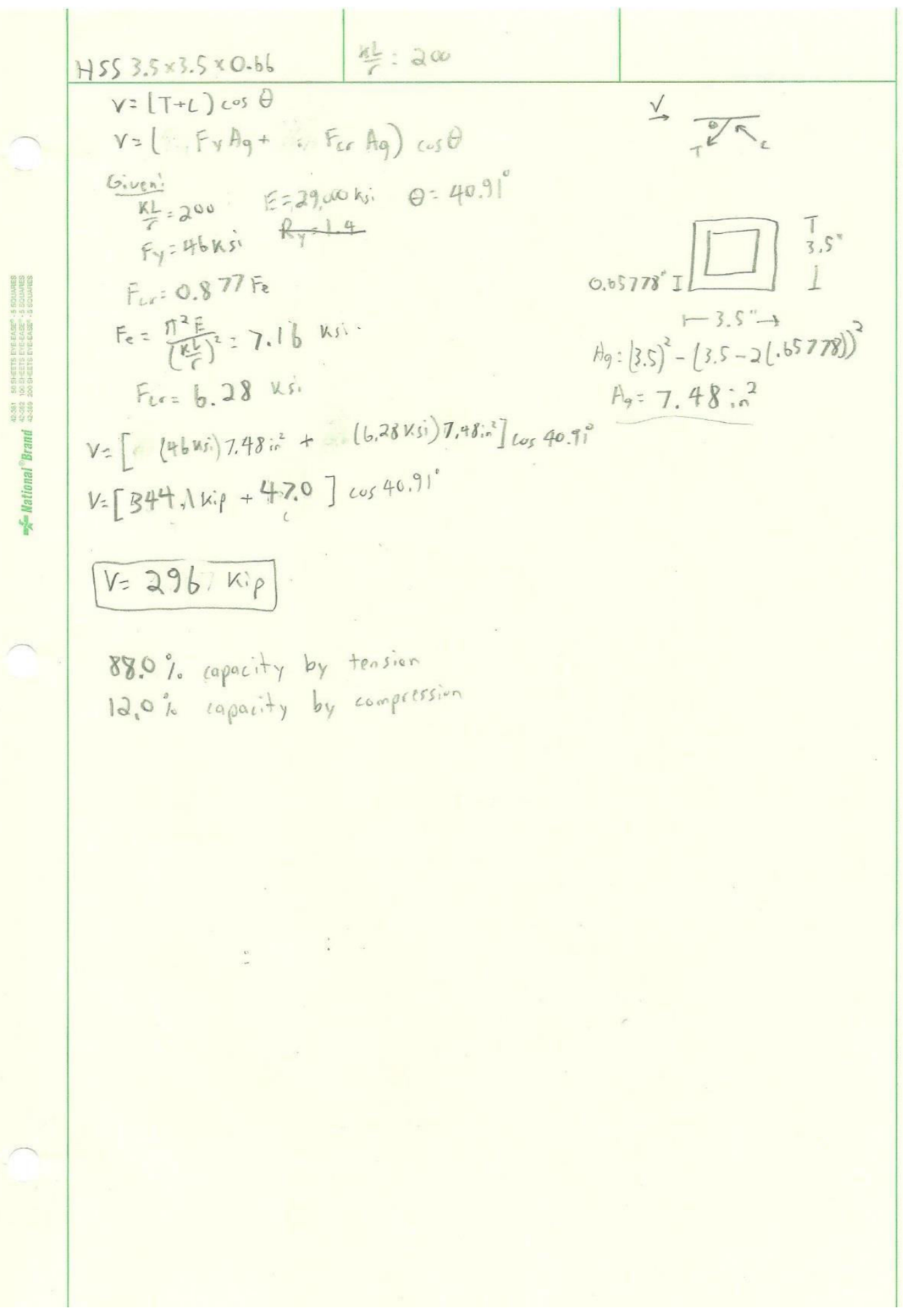




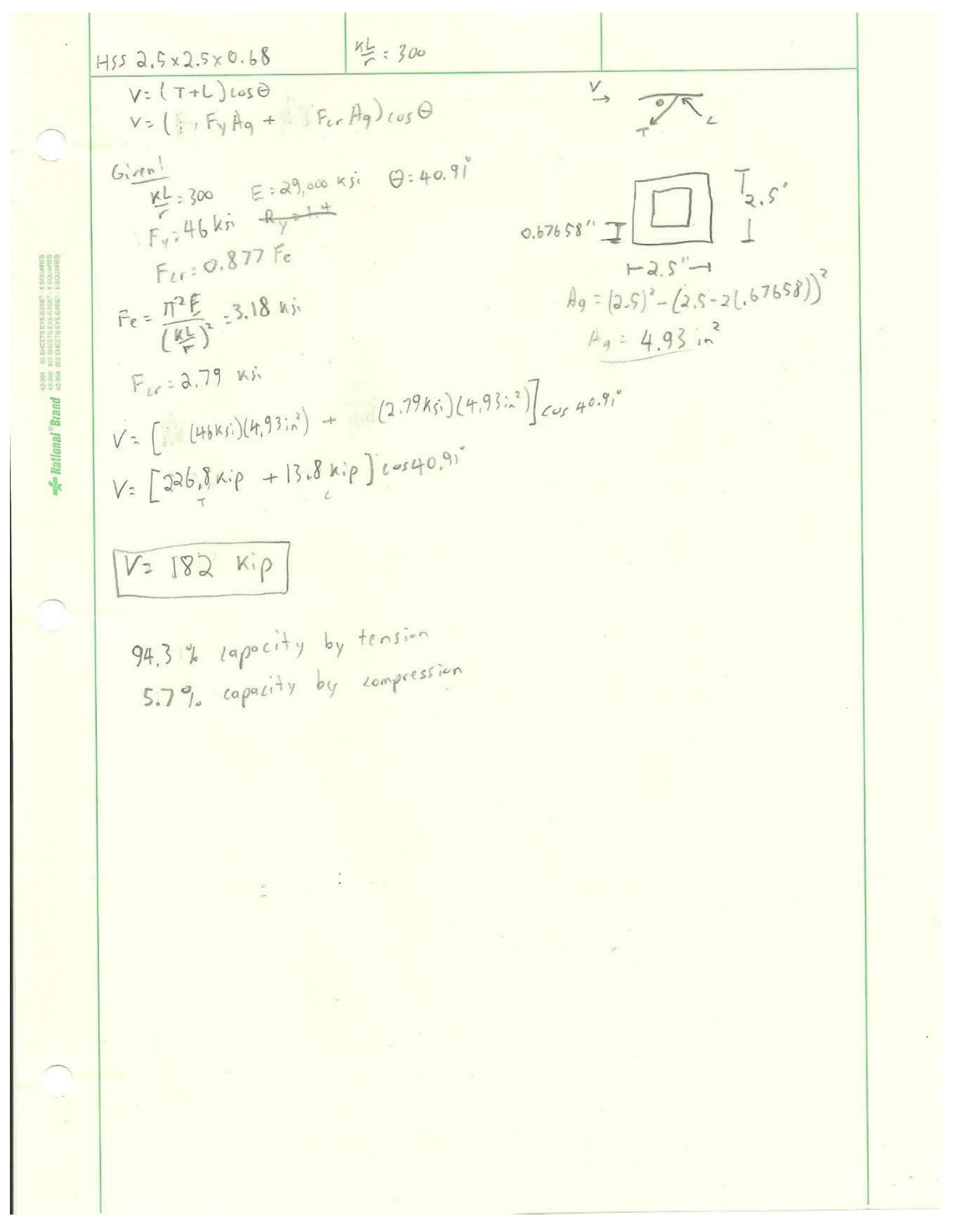


Initial Scaling Factor

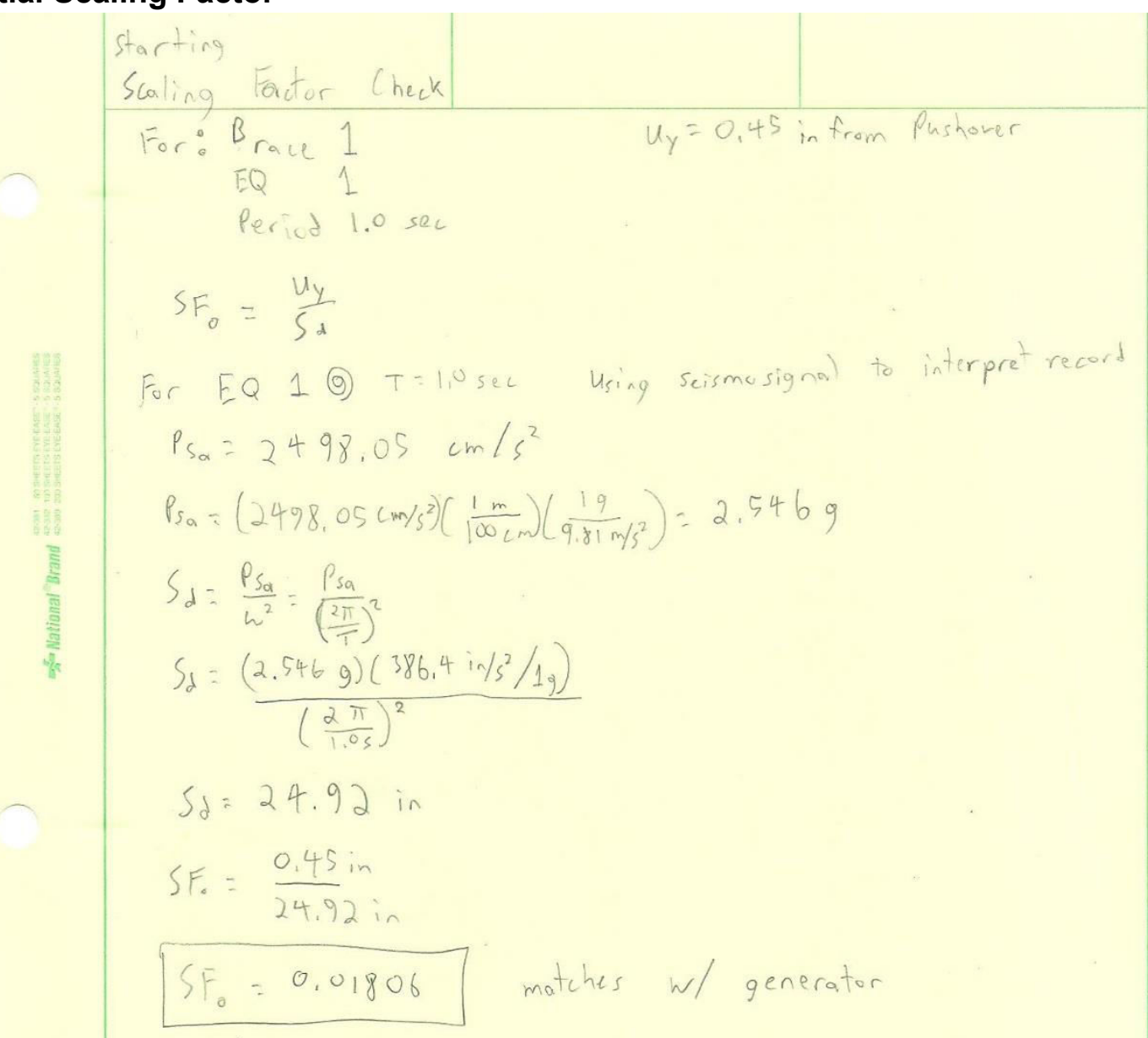




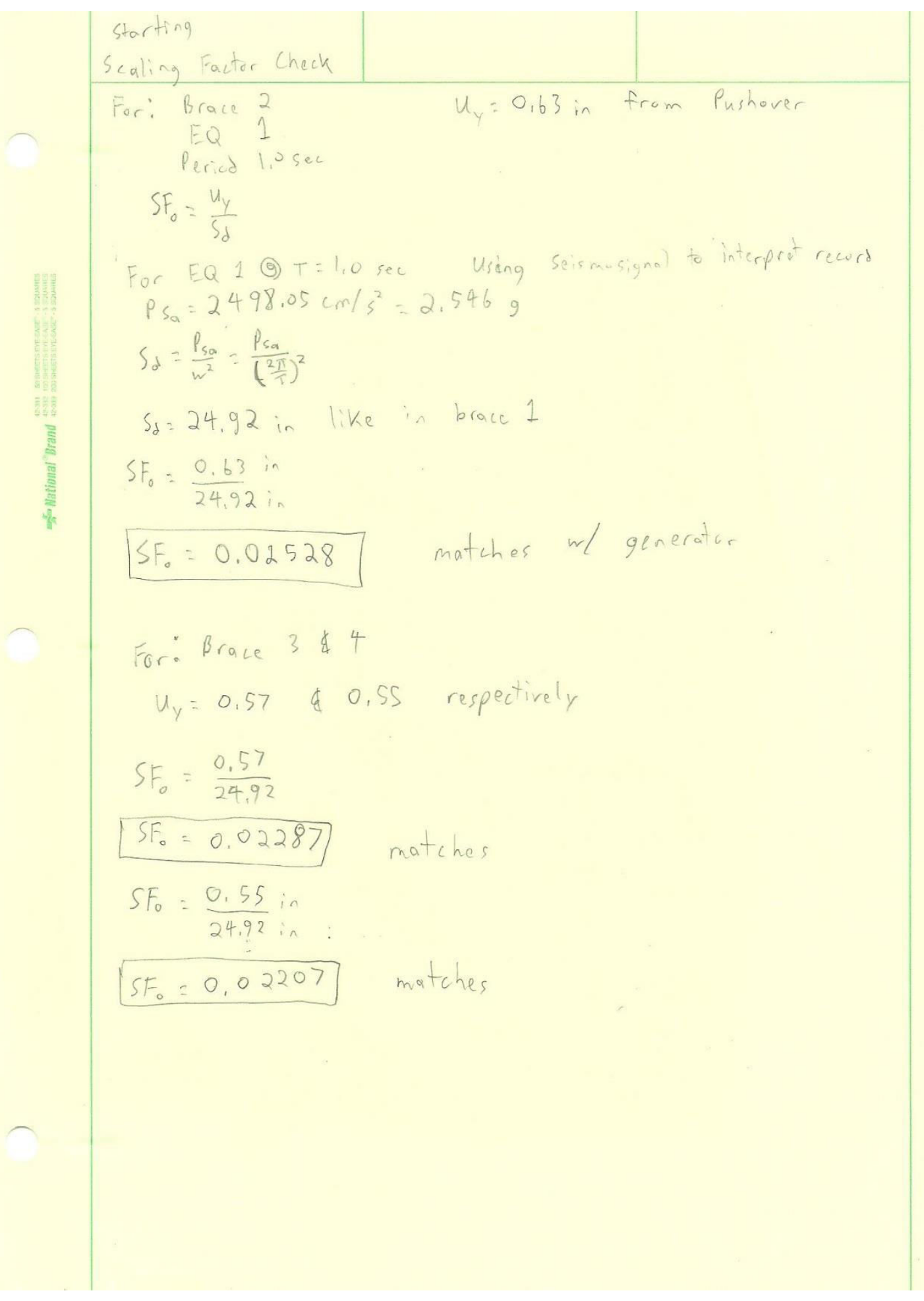


F. AISC Seismically Compact HSS Square Hollow Sections

Table F-1: Seismically Compact HSS Square Hollow Sections.

\begin{tabular}{|c|c|c|c|c|c|}
\hline Shape & $\boldsymbol{b} / \boldsymbol{t}_{\text {des }}$ & $\boldsymbol{K}$ KL/r & Shape & $\boldsymbol{b} / \boldsymbol{t}_{\text {des }}$ & $\mathbf{K L / r}$ \\
\hline HSS10X10X5/8 & 14.2 & 61.82839 & HSS4X4X3/8 & 8.45 & 159.08542 \\
HSS9X9X5/8 & 12.5 & 69.13933 & HSS4X4X5/16 & 10.8 & 156.82958 \\
HSS8X8X5/8 & 10.8 & 78.40511 & HSS4X4X1/4 & 14.2 & 154.59398 \\
HSS8X8X1/2 & 14.2 & 77.2874 & HSS3-1/2X3-1/2X3/8 & 7.02 & 184.04386 \\
HSS7X7X5/8 & 9.05 & 90.52703 & HSS3-1/2X3-1/2X5/16 & 9.04 & 181.07951 \\
HSS7X7X1/2 & 12.0 & 89.05845 & HSS3-1/2X3-1/2X1/4 & 12.0 & 178.14209 \\
HSS6X6X5/8 & 7.33 & 107.0573 & HSS3X3X3/8 & 5.59 & 218.2109 \\
HSS6X6X1/2 & 9.91 & 105.0443 & HSS3X3X5/16 & 7.32 & 214.1495 \\
HSS6X6X3/8 & 14.2 & 103.0541 & HSS3X3X1/4 & 9.87 & 210.12316 \\
HSS5-1/2X5-1/2X3/8 & 12.8 & 113.0137 & HSS3X3X3/16 & 14.3 & 206.07418 \\
HSS5-1/2X5-1/2X5/16 & 15.9 & 111.8372 & HSS2-1/2X2-1/2X5/16 & 5.60 & 261.86995 \\
HSS5X5X1/2 & 7.74 & 127.9879 & HSS2-1/2X2-1/2X1/4 & 7.73 & 256.02599 \\
HSS5X5X3/8 & 11.3 & 125.0984 & HSS2-1/2X2-1/2X3/16 & 11.4 & 250.14735 \\
HSS5X5X5/16 & 14.2 & 123.6691 & HSS2-1/4X2-1/4X1/4 & 6.65 & 287.36438 \\
HSS4-1/2X4-1/2X1/2 & 6.67 & 143.6511 & HSS2-1/4X2-1/4X3/16 & 9.94 & 280.07214 \\
HSS4-1/2X4-1/2X3/8 & 9.89 & 140.0668 & HSS2X2X1/4 & 5.58 & 327.36905 \\
HSS4-1/2X4-1/2X5/16 & 12.5 & 138.2939 & HSS2X2X3/16 & 8.51 & 318.09274 \\
HSS4X4X1/2 & 5.59 & 163.645 & HSS2X2X1/8 & 14.2 & 309.11127 \\
\hline
\end{tabular}

